Summer 2016

\title{
Thin Film Approaches to The Srf Cavity Problem: Fabrication and Characterization of Superconducting Thin Films
}

Douglas Beringer

College of William and Mary - Arts \& Sciences, beringerd@gmail.com

Follow this and additional works at: https://scholarworks.wm.edu/etd

Part of the Physics Commons

\section{Recommended Citation}

Beringer, Douglas, "Thin Film Approaches to The Srf Cavity Problem: Fabrication and Characterization of Superconducting Thin Films" (2016). Dissertations, Theses, and Masters Projects. Paper 1499449840. http://doi.org/10.21220/S2S087

This Dissertation is brought to you for free and open access by the Theses, Dissertations, \& Master Projects at W\&M ScholarWorks. It has been accepted for inclusion in Dissertations, Theses, and Masters Projects by an authorized administrator of W\&M ScholarWorks. For more information, please contact scholarworks@wm.edu. 
Thin Film Approaches to the SRF Cavity Problem:

Fabrication and Characterization of Superconducting Thin Films

Douglas B. Beringer

Billings, Montana

Master of Science, College of William and Mary, 2009

Bachelor of Arts, Reed College, 2004

A Dissertation presented to the Graduate Faculty

of the College of William and Mary in Candidacy for the Degree of Doctor of Philosophy

Department of Physics

The College of William and Mary

January 2017 
(C) 2017

Douglas B. Beringer

All rights reserved. 


\section{APPROVAL PAGE}

This Dissertation is submitted in partial fulfillment of the requirements for the degree of

Doctor of Philosophy

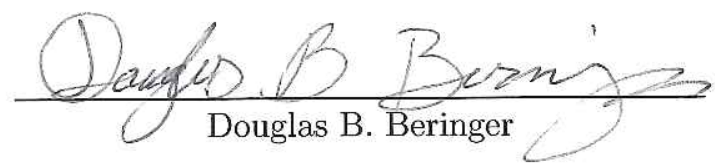

Approved by the Committee, September, 2016

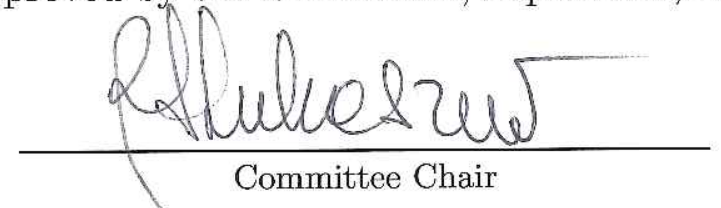

VMEC Distinguished Professor R. Alejandra Lukaszew, Physics The College of William and Mary

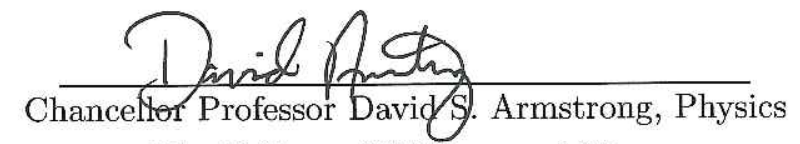

The College of William and Mary

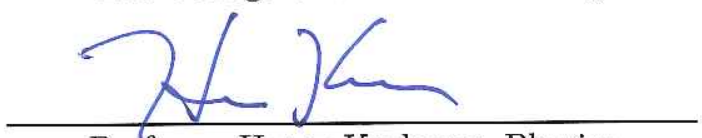

Professor Henry Krakauer, Physics

The College of William and Mary

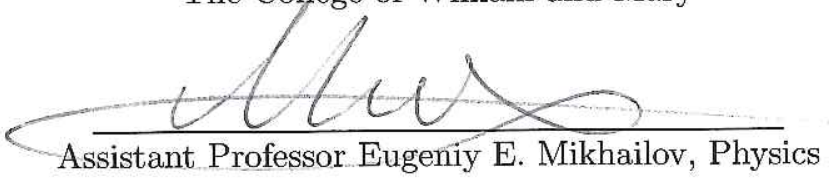

The College of William and Mary

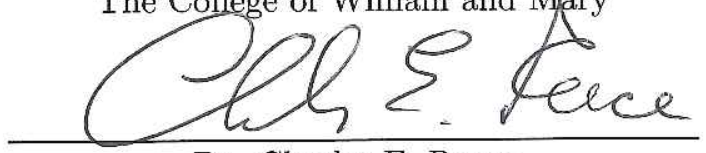

Dr. Charles E. Reece

Jefferson Lab Institute for SRF Science and Technology 


\begin{abstract}
Superconducting Radio Frequency (SRF) cavities are responsible for the acceleration of charged particles to relativistic velocities in most modern linear accelerators, such as those employed at high-energy research facilities like Thomas Jefferson National Laboratory's CEBAF and the LHC at CERN. Recognizing SRF as primarily a surface phenomenon enables the possibility of applying thin films to the interior surface of SRF cavities, opening a formidable tool chest of opportunities by combining and designing materials that offer greater performance benefit. Thus, while improvements in radio frequency cavity design and refinements in cavity processing techniques have improved accelerator performance and efficiency - 1.5 GHz bulk niobium SRF cavities have achieved accelerating gradients in excess of $35 \mathrm{MV} / \mathrm{m}$ - there exist fundamental material bounds in bulk superconductors limiting the maximally sustained accelerating field gradient $(\approx 45 \mathrm{MV} / \mathrm{m}$ for $\mathrm{Nb})$ where inevitable thermodynamic breakdown occurs. With state of the art $\mathrm{Nb}$ based cavity design fast approaching these theoretical limits, novel material innovations must be sought in order to realize next generation SRF cavities. One proposed method to improve SRF performance is to utilize thin film superconducting-insulating-superconducting (SIS) multilayer structures to effectively magnetically screen a bulk superconducting layer such that it can operate at higher field gradients before suffering critically detrimental SRF losses. This dissertation focuses on the production and characterization of thin film superconductors for such SIS layers for radio frequency applications. Correlated studies on structure, surface morphology and superconducting properties of epitaxial $\mathrm{Nb}$ and $\mathrm{MgB}_{2}$ thin films are presented.
\end{abstract}




\section{TABLE OF CONTENTS}

Acknowledgments ................................. iv

Dedication . . . . . . . . . . . . . . . . . . . . v

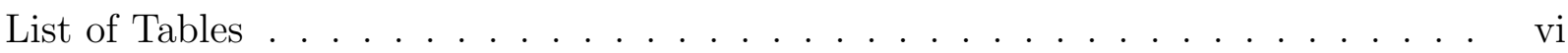

List of Figures . . . . . . . . . . . . . . . . . . . . vii

\section{CHAPTER}

1 Introduction ........................... 1

1.1 Superconducting Radio Frequency Cavities . . . . . . . . . . . . . . 2

1.2 Thin Film SIS Multilayers . . . . . . . . . . . . . . . 9

1.3 Scope of Dissertation . . . . . . . . . . . . . . . . 11

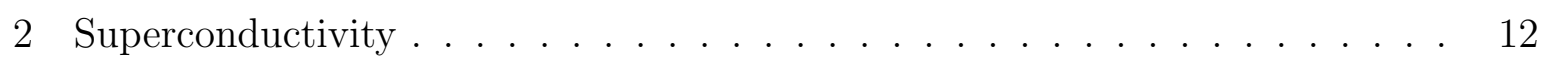

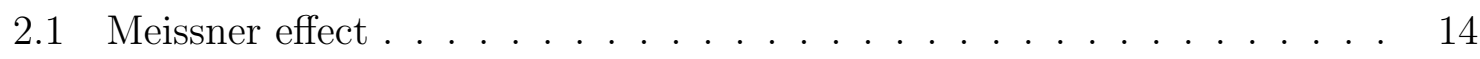

2.2 London Theory . . . . . . . . . . . . . . . . . . 16

2.3 BCS in brief . . . . . . . . . . . . . . . . . 19

2.4 Two-Fluid Model and Surface Resistance of Superconductors . . . . . . 22

2.5 Thin Film Superconductors . . . . . . . . . . . . . . 23

3 Thin Film Growth and Scaling in Dynamic Systems . . . . . . . . . . . . . 25

3.1 Nucleation and Growth of Thin Films . . . . . . . . . . . . . . . 26

3.2 Fractals, Self-similarity and Scaling . . . . . . . . . . . . . 31

3.2.1 Family-Vicsek Scaling . . . . . . . . . . . . . . . 33

3.2.2 General Dynamic Scaling . . . . . . . . . . . . . . . . 34 
4 Experimental Methods . . . . . . . . . . . . . . . . . . . . . 37

4.1 Thin Film Deposition . . . . . . . . . . . . . . . . . 38

4.1.1 Vacuum Technology and Thin Films . . . . . . . . . 40

4.1 .2 DC Magnetron Sputtering . . . . . . . . . . . . . . 41

4.1.3 Pulsed Sputtering and Reactive Sputtering . . . . . . . . . . . 44

4.1.4 Hybrid Physical-Chemical Vapor Deposition of $\mathrm{MgB}_{2}$ Thin Films 45

4.2 Thin Film Characterization _ . . . . . . . . . . . . . . 46

4.2 .1 Atomic Force Microscopy . . . . . . . . . . . . . . . . 47

$4.2 .2 \quad$ Diffraction Techniques . . . . . . . . . . . . . . . . . . . . 49

4.3 Magnetic Characterization via SQUID . . . . . . . . . . 56

4.3.1 Determining $T_{C}$ and $H_{C 1}$ of Superconducting Thin Films $\ldots . \quad 58$

5 Structure and Property Correlations in Superconducting Thin Films . . . . . 61

5.1 Epitaxial $\mathrm{Nb}$ Grown on $\mathrm{MgO}(001)$ Surfaces . . . . . . . . . . . . . . . 61

$5.1 .1 \quad$ Experimental Details . . . . . . . . . . . . . . . 63

5.1.2 Characterization of Structure and Morphology . . . . . . . . . 65

5.1.3 Determination of Scaling Exponents . . . . . . . . . . . . . . 69

5.2 Strain Effects on the Crystal Growth and Superconducting Properties of Epitaxial Nb Ultrathin Films . . . . . . . . . . . . . . 76

5.2.1 Epitaxial $\mathrm{Nb}$ Thin films on $a$-plane $\mathrm{Al}_{2} \mathrm{O}_{3} \quad \ldots \ldots \ldots \ldots$

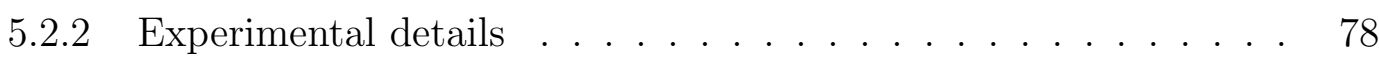

5.2.3 Surface Morphology of $\mathrm{Nb} / \mathrm{Al}_{2} \mathrm{O}_{3}$ Thin Films . . . . . . . . . 79

5.2.4 Structural Characterization of Nb/Al2O3 Thin Films . . . . . 80

5.2 .5 Superconducting Measurements . . . . . . . . . . . . 86

$5.3 \mathrm{MgB}_{2}$ Thin Films . . . . . . . . . . . . . . . . . . . . . . . . 92

$5.3 .1 \mathrm{MgB}_{2}$ Experimental Details . . . . . . . . . . . . . 93 
5.3.2 Structural Characterization _. . . . . . . . . . . . . 94

5.3.3 Morphology of $\mathrm{MgB}_{2}$ Thin Films . . . . . . . . . . . . . 96

5.3.4 $\mathrm{H}_{C 1}$ Measurements on $\mathrm{MgB}_{2} \ldots \ldots . \ldots 96$

5.3.5 $\mathrm{MgB}_{2}$ Summary . . . . . . . . . . . . . . . . 98

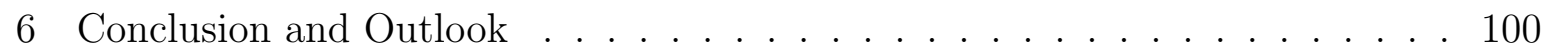

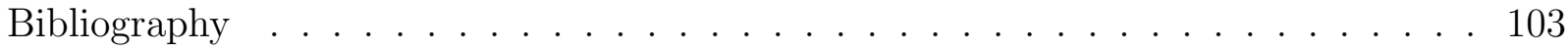




\section{ACKNOWLEDGMENTS}

I am grateful to the many people who have supported me throughout the course this research and my graduate education. I would first like to thank my advisor, Ale Lukaszew, for her mentorship, insight conversations and unstoppable dedication. I would also like to thank and acknowledge current and former members of the Lukaszew research group at the College of William and Mary for a countable multitude of contributions: William Roach, César Clavero, Jonathan Skuza, Kaida Yang, Lei Wang, Zhaozhu Li, Melissa Beebe, Matthew Burton and José Riso. I would like to thank Teng Tan and Xiaoxing Xi of Temple University for material contribution of high quality $\mathrm{MgB}_{2}$ samples to this research project. Thanks also to Stuart Wolf and Jiwei Lu of the University of Virgina as well as our collaborators within the SRF community at Jlab, particularly Larry Phillips, Anne-Marie Valente-Feliciano and Charlie Reece. Additional thanks to Diefang Gu, A. D. Batchelor and K. C. Wong for their work with TEM on the $\mathrm{Nb} / \mathrm{Al}_{2} \mathrm{O}_{3}$. Thanks to Nate Phillips, Kelly Klutz, Megan Ivory, Zachariah DeMeola, Aria Johansen, Eric Walter and Stan for supporting me and sharing a home with me at various intervals throughout my graduate school career. This research was made possible by financial support from the U. S. Department of Energy (DOE: DE- AC05-06OR23177) and the Defense Threat Reduction Agency (DTRA: HDTRA1-10-1-0072). 
For my Mother and Father. 


\section{LIST OF TABLES}

1.1 Comparison of candidate superconducting materials. . . . . . . . . . . 10

5.1 Summary of superconducting properties of Nb thin films. . . . . . . . . 75

5.2 Comparison of in-plane $H_{C 1}$ and $H_{C 2}$ values . . . . . . . . . . . . . . . 91 


\section{LIST OF FIGURES}

$1.1 \mathrm{Nb} \mathrm{SRF}$ accelerating cavity. . . . . . . . . . . . . . . . 3

1.2 Simplified representation of a SRF cavity. . . . . . . . . . . . . . . 4

1.3 Schematic cross section illustrating magnetic field geometry at the interior SRF surface. . . . . . . . . . . . . . . . 6

1.4 Sketch of Cavity Q as a function of accelerating field . . . . . . . . . 8

1.5 Schematic Illustration of proposed SIS superstructures . . . . . . . . . . . 10

2.1 Resistivity as a function of temperature for conventional and superconducting materials. . . . . . . . . . . . . . . . . . . . . . . . . 13

2.2 Dielectric response of superconductors . . . . . . . . . . . . . 15

2.3 Representation of magnetic vortices in a Type-II superconductor. . . . . . 16

3.1 Modified SZD diagram . . . . . . . . . . . . . . . . . . 29

3.2 Cross-sectional illustration of a hypothetical multilayer coating . . . . . . . 30

3.3 Iterative construction of a Koch snowflake . . . . . . . . . . . . . . 33

4.1 Illustration of a magnetron sputtering system. . . . . . . . . . . . . . 43

4.2 Schematic illustrating a reactive magnetron sputtering process. . . . . . . 45

4.3 Illustration of an AFM configuration. . . . . . . . . . . . . . . 48

4.4 Grazing-incidence geometry in a typical RHEED experiment. . . . . . . . . 51

4.5 Four circle goniometer . . . . . . . . . . . . . . . . . 54

4.6 DC SQUID device. . . . . . . . . . . . . . . . . . 57

4.7 Geometry of MPMS SQUID experiment. . . . . . . . . . . . . . 60 
$5.1 \mathrm{Nb} / \mathrm{MgO}$ epitaxial relationships as verified by RHEED. . . . . . . . . . . 66

5.2 Representative $2 \times 2 \mu \mathrm{m} \mathrm{AFM} \mathrm{scans} \mathrm{of} \mathrm{Nb} \mathrm{thin} \mathrm{films} \mathrm{from} \mathrm{Series} \mathrm{1} \mathrm{\ldots} \mathrm{.} 67$

5.3 Representative $2 \times 2 \mu \mathrm{m}$ AFM scans of Nb thin films from Series $2 \ldots$

5.4 Logarithmic scaling plots from Series 1 films . . . . . . . . . . . . . 71

5.5 Power Spectral Density curves for Series 1 Nb films. . . . . . . . . . . . 72

5.6 Logarithmic plots for $\mathrm{Nb}$ thickness Series 2. . . . . . . . . . . . 73

5.7 Resistivity as a function of temperature . . . . . . . . . . . . 74

5.8 AFM topography images for $\mathrm{Nb}$ films grown on $a$-plane sapphire . . . . . . 81

5.9 Evolution of the $\mathrm{Nb}$ structure and lattice parameter for $\mathrm{Nb}$ films . . . . . 83

5.10 Symmetric XRD scans for $\mathrm{Nb}$ films grown on $a$-plane sapphire $\ldots . . . .85$

5.11 TEM image of $\mathrm{Al}_{2} \mathrm{O}_{3}(11 \overline{2} 0) / \mathrm{Nb}(110)$ interface. . . . . . . . . . . . 87

5.12 AC susceptibility curves for $\mathrm{Nb}$ thin films . . . . . . . . . . . . . . . 89

5.13 In-plane magnetization vs. in-plane applied magnetic field for Nb films . . 90

5.14 X-ray diffraction of $\mathrm{MgB}_{2}$ on $c$-plane sapphire. . . . . . . . . . 95

5.15 AFM images of $\mathrm{MgB}_{2}$ thin films. . . . . . . . . . . . . 97

5.16 Measured $\mathrm{H}_{C 1}$ vs. $\mathrm{MgB}_{2}$ film thickness . . . . . . . . . . . . 98 


\section{CHAPTER 1}

\section{Introduction}

Particle accelerators in research are primary experimental tools used in the generation and interrogation of elementary particles. Advances in superconducting materials and technology parallel the increased accessibility to the high center of mass energies required by many electron, proton, antiproton, and heavy ion accelerators. The Tevatron at Fermi National Accelerator Laboratory (FNAL) was made possible with the successful design and utilization of superconducting NbTi dipole and quadrupole magnets. Steady material and design improvements in superconducting radio frequency (SRF) cavities have resulted in electron beam facilities achieving very high beam energies, like the Continuous Electron Beam Accelerator Facility (CEBAF) at Thomas Jefferson National Accelerator Facility. While many linear accelerators used worldwide are made from conventional conducting materials, such as $\mathrm{Cu}$, it is the SRF cavity that enables economical continuous wave operation with accelerating gradients in excess of $35 \mathrm{MV} / \mathrm{m}$ [1].

Radio frequency cavities made from conventional conductors (e.g. $\mathrm{Cu}$ ) have been in development since the early 20th century. With the rise of superconductivity and the increased availability of the relatively new commodity of liquid helium $\left(\mathrm{L}_{H e}\right)$ in the $1950 \mathrm{~s}$, 
interest in and research on SRF technology accelerated. The first SRF resonator cavities were proposed in 1961 by W. Fairbank of Stanford University. In 1964, the Stanford research group showcased the first SRF electron accelerator - a Cu cavity electroplated with superconducting $\mathrm{Pb}$ with quality factors approaching $10^{8}[2]$.

Niobium $(\mathrm{Nb})$ is currently the material of choice for most modern SRF cavities because — in addition to being relatively easy to refine, purify, and machine - $\mathrm{Nb}$ is an abundant material with the highest superconducting transition temperature $T_{C} \approx 9.2 \mathrm{~K}$ and the highest lower critical field $\left(H_{C 1} \approx 190 \mathrm{mT}\right)$ of the pure element superconductors.

The path to high performance SRF cavities has been marked with steady improvements in cavity design, material processing and cryogenics, the culmination of which are bulk Nb SRF cavities performing near theoretical material limits. These advancements stand as a testament to the dedication and innovation of the SRF community over the decades. Recent advances in thin film coatings have produced accelerator cavities somewhat comparable to bulk $\mathrm{Nb}$ standards. A comprehensive history of the many developments leading to the current state of the art in radio frequency superconductivity, while deep and fascinating, is beyond the scope of this thesis; however an interested reader may find more information in reference [3].

The research contained in this thesis represents an effort to develop and characterize new materials and approaches to improve the SRF surfaces for accelerator cavities. By way of introduction, this chapter will first sketch the most salient features of SRF cavities and introduce the practical limitations next generation materials need to address.

\subsection{Superconducting Radio Frequency Cavities}

Superconducting radio frequency cavities offer several advantages over resonant cavities based on conventional conductors. Comparatively, superconducting resonators provide 
large gains in the quality factor $Q_{0}$, a dimensionless parameter common to all resonators defined as the ratio of the stored energy in the cavity to the energy lost in one cycle. Typical quality factors of Nb-based superconducting cavities, like the one pictured in Figure 1.1 , used in linear accelerators are in the range of $10^{9}$, but some in excess of $10^{11}$ have been reported - among the highest $Q$ values recorded in any system[4]. The microwave surface resistance for SRF cavities is much lower than those for conventional cavities, hence nearly all of the radio frequency power is delivered to the beam while the power dissipation in the walls of SRF cavities is minimal. Thus, for applications where large accelerating gradients are required (linear accelerators, storage rings, etc.), SRF cavities (including considerable cryogenic costs and accommodation) offer clear performance and economic advantages over conventional radio frequency cavities, particularly when operating continuously at higher accelerating fields [3].

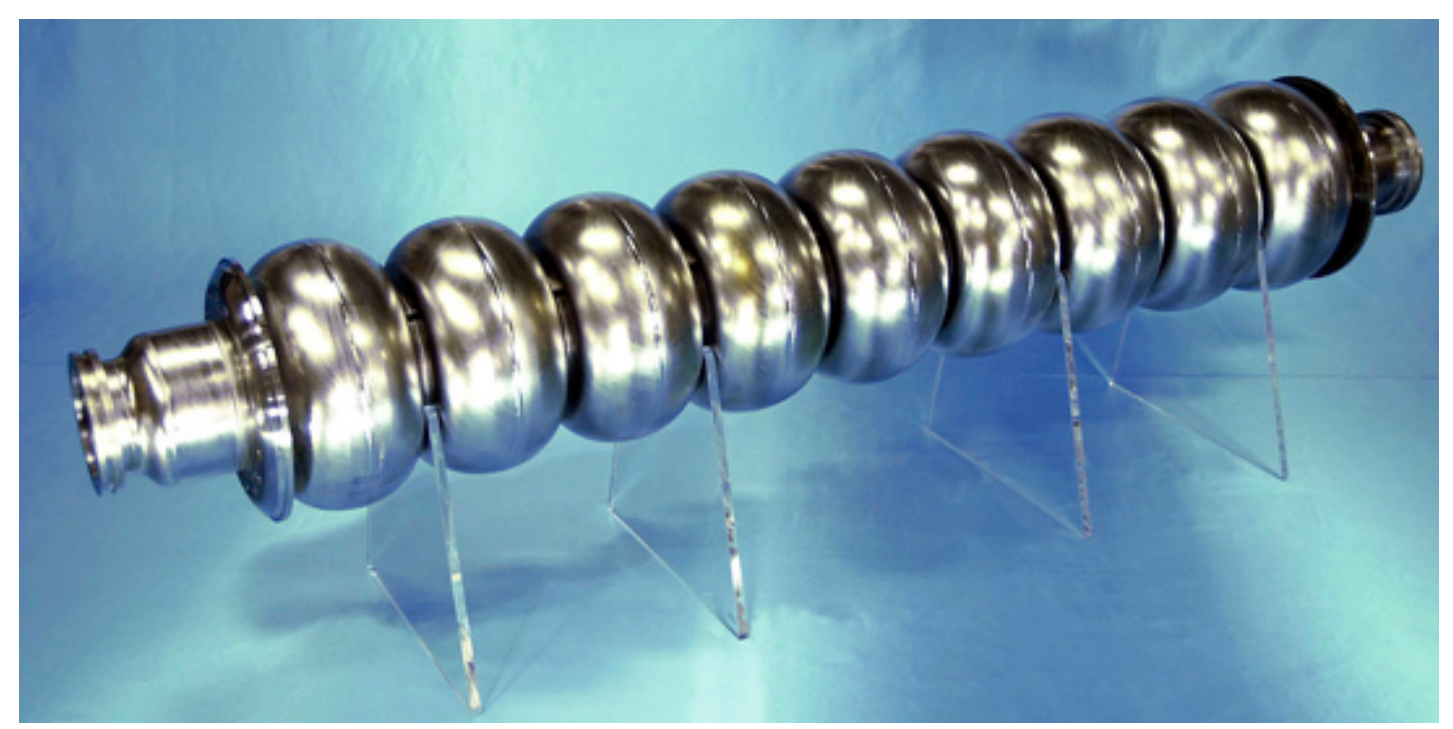

FIG. 1.1: Nb SRF accelerating cavity. Photo credit jlab.org.

A simplified representation of an SRF accelerator cavity is pictured in Figure 1.2. The resonator cavity, typically a Nb structure with a wall thickness of a few millimeters, 
is completely immersed in a liquid helium bath to maintain the superconducting phase. Driving power is provided by an antennae coupling a radio frequency source to the accelerating fields produced within the cavity. The characteristic bandwidth $\Delta \omega$ is related to the $\mathrm{Q}$ of the SRF cavity and a characteristic resonant frequency $\omega_{0}$ by

$$
\Delta \omega=\frac{\omega_{0}}{2 Q}
$$

While high-Q resonators operate over a very narrow frequency range, the presence of higher-order resonances can be problematic for continuous operation. To mitigate these issues, additional resonating structures, such as waveguides, are coupled to the SRF cavity in order to filter out and attenuate detrimental higher-order resonances during operation.

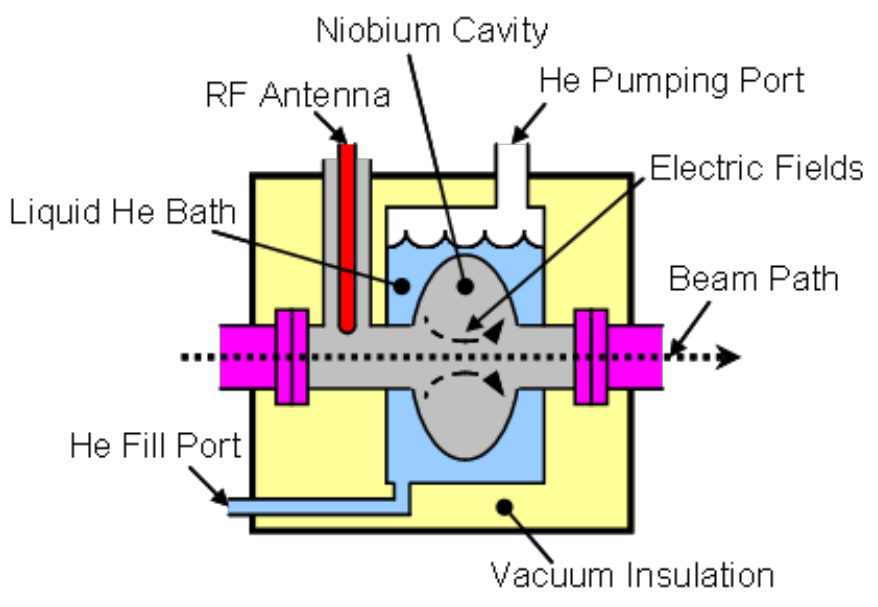

FIG. 1.2: Simplified representation of a SRF cavity. The accelerator cavity is completely immersed in a $\mathrm{L}_{H e}$ bath maintained around $2 \mathrm{~K}$ via pumping. Charged particles are accelerated along the length of the cavity.

In the context of SRF cavities, an uncontrolled transition of the superconducting material to normal state can be catastrophic. As such, thermal transport and heat dissipation must be carefully considered. For materials in a normal state, electronic interactions are 
the primary mechanisms responsible for thermal conductivity in materials. In Type-II superconductors however, as increasing proportions of the conduction electrons are bound as Cooper pairs, thermal conductivity will be increasingly dominated by acoustic mechanisms, hence an overall decrease in thermal conductivity of superconductors.

As such, the refrigeration system is a critical component for the successful operation of SRF cavities. Superconducting cavities experience radio frequency heating; hence, the $\mathrm{L}_{\mathrm{He}}$ reservoir must efficiently draw thermal energy from the walls of the cavity to maintain the superconducting state. Owing to a low Carnot efficiency at $\mathrm{L}_{H e}$ temperatures, the refrigeration systems must pump that thermal energy away at a sufficient rate to prevent thermal breakdown and quenching. In order to combat this thermodynamic reality, the pressure and temperature of the helium bath are often maintained below the $\lambda$-point of $\mathrm{He}_{4}$ (around $2.17 \mathrm{~K}$ ) to take advantage of the higher heat capacity of superfluid helium and a corresponding increase of thermal conductance. Superfluid helium offers additional benefits for this application, as superfluid helium only boils at free interfaces; consequently, the Nb-He interface is calm, minimizing local, inhomogeneous regions of heating or cooling which might encourage mechanical and thermodynamic failures.

Additionally, the maximum accelerating field within the cavity is limited by the maximum $\mathbf{E}$ and $\mathbf{H}$ fields that the interior SRF surface can withstand before incurring critical breakdowns. High electric fields proximal to the SRF surface can give rise to field emission effects; thus, sufficiently sharp morphological features or surface contamination can serve as field emission sites, releasing stray electrons into the accelerator cavity during operation. These off-axis electrons may then be accelerated and subsequently impinge upon the cavity surface, causing local heating or even a cascading release of further electrons whose collisions incite thermal instability, production of significant amounts of ionizing radiation and quenching.

Careful control of the SRF surface necessitates that the production of SRF cavities 
occur in industrial clean room environments, like those found at JLab fabrication facilities, to minimize surface defects and contamination. Many surface processing treatments including electropolishing, high pressure washes, and vacuum bakes are used to minimize the roughness of the surface features and eliminate external contaminants on the SRF surface.

The primary accelerating mode in most modern SRF cavities is the fundamental $\mathrm{TM}_{010}$ mode. In this mode, it is important to note that the microwave magnetic field $\mathbf{H}$ is oriented parallel to the SRF surface and achieves its maximum at the interior cavity wall. Consequently, conservative operation would dictate that for the cavity to maintain the superconducting state the $H$ field must remain lower than the critical field $H_{C 1}$ for the superconductor; however, in practice radio frequency operation in the Meissner state may persist at $H_{r m f}>H_{C 1}$.

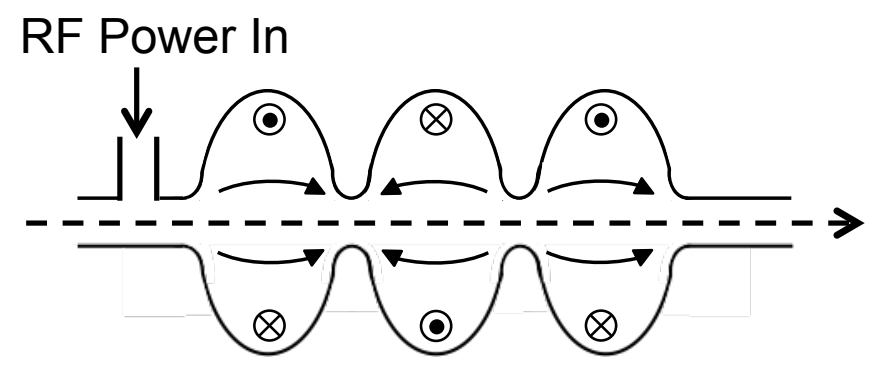

FIG. 1.3: Schematic cross section illustrating magnetic field geometry at the interior SRF surface.

For Type-II superconductors, it is possible for the Meissner state to persist thermodynamically in a metastable superheated state up to $H_{s h} \leq H_{C 2}$, above which fluxoids form and the superconductor enters the mixed state. Indeed, it has been demonstrated that real SRF cavities can operate persistently near the theoretical maximum $H_{s h}$ before 
suffering catastrophic breakdown. The nucleation of magnetic fluxoids (corresponding to a collapse of the Meisnner state) in Type-II superconductors occurs on time scales on the order of $10^{-6} \mathrm{~s}$ - long in comparison to a typical radio frequency period (say on the order of $10^{-9} \mathrm{~s}$ ). Thus, in radio frequency operation, a metastable superheated state is possible, where vortex penetration is suppressed for $H_{s h}$ fields greater than $H_{C 1}$.

Thus, a primary limitation of SRF cavities is given by the requirement that the radio frequency magnetic field at the interior surface has to stay below $H_{s h}$ of the superconductor, corresponding to a maximum accelerating field of $E_{a c c}=45 \mathrm{MV} / \mathrm{m}$ for an ideal $\mathrm{Nb}$ cavity. In principle, the quality factor should stay constant when approaching this fundamental superconductor limit; in practice however, a decay of the quality factor with increasing accelerating field, or $Q$-slope, is observed. This suppression of the quality factor at high accelerating gradients is attributable to material and surface imperfections. Figure 1.4 qualitatively shows $\mathrm{Q}$ as a function of accelerating electric field for ideal and real (coated and uncoated) SRF cavities.

In addition to material considerations, SRF performance is also extremely sensitive to the particular geometry of the resonator cavity. Advances in cavity design, composition, cleaning, polishing, and processing now yield bulk Nb cavities operating near theoretical material limits. Notably, the problem of multipacting - a phenomenon whereby a resonant cascade of charge over a small region of the SRF surface promotes local heating - has been rectified by improvements in cavity geometry. An interested reader may find a nice introduction to ancillary topics related to SRF design in [3].

There remain myriad material and design challenges in order to overcome fundamental limitations of bulk SRF cavities. Novel material solutions are currently sought in order to improve performance, including the development of thin film coated $\mathrm{Cu}$ radio frequency cavities using more exotic superconducting compounds with more desirable superconducting and thermal properties. Thin film approaches also introduce new sets of challenges, 


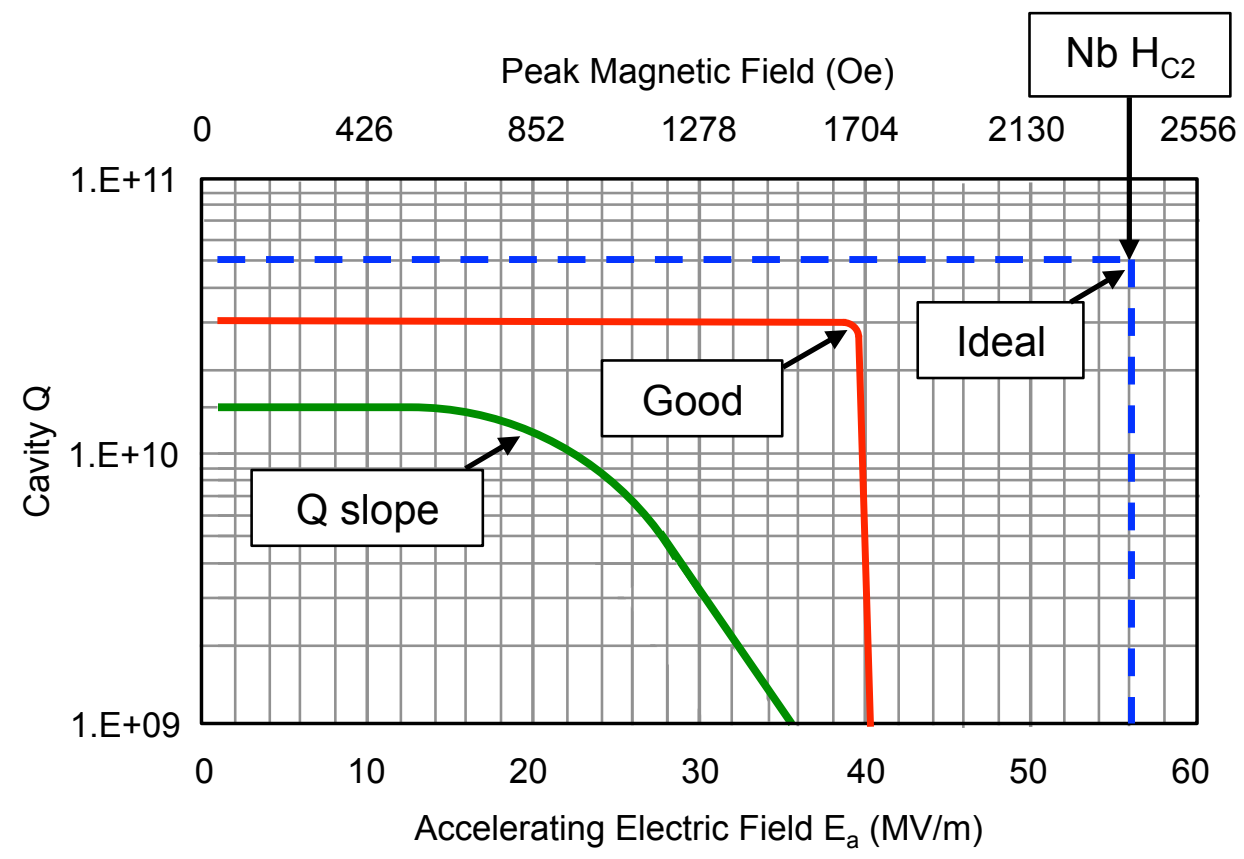

FIG. 1.4: Sketch of Cavity Q as a function of accelerating field in SRF accelerator cavities. 
particularly in the large-scale, industrial realization of thin film coated SRF cavities including issues with conformal coverage, ensuring the control of appropriate microstructure and morphology of thin films, etc. Despite these technical challenges, thin film approaches remain a promising research avenue for SRF materials.

\subsection{Thin Film SIS Multilayers}

Bulk Nb SRF cavities are approaching the theoretical upper limits for achievable accelerating gradients $(\sim 45 \mathrm{MV} / \mathrm{m})$ according to the constraints dictated by the maximum critical field of bulk Nb cavities ( $H_{s h} \approx 210 \mathrm{mT}$ ); thus, novel solutions are required to push the performance of SRF cavities further. A "simple" change in superconducting material alone will not suffice, especially since bulk $\mathrm{Nb}$ is already an attractive material for SRF applications (well-studied, machinable, pure elemental superconductor, high lower critical field, and large superconducting energy gap).

One proposed scheme, by A. Gurevich, suggests a method by which an SRF surface may effectively operate at even higher accelerating field gradients via clever application of thin films [5]. The seminal idea is to use multilayer SIS (superconducting-insulatingsuperconducting) thin film superstructures deposited on the inner cavity surface to delay vortex penetration (effectively increasing $H_{s h}$ ) while tailoring SRF surfaces to minimize heating due to surface resistance (so as to delay or reduction in Q).

A thin film Type-II superconductor with $T_{C}$ and $H_{s h}$ greater than that of $\mathrm{Nb}$ is desired to serve as magnetic attenuation layers to inhibit the onset of vortex penetration in a bulk superconducting material. Very thin intercalated insulating layers serve to decouple the superconducting surfaces. Table 1.1 lists fundamental bulk superconducting properties of potential candidate superconducting compounds alongside $\mathrm{Nb}$ [6].

Figure 1.5 shows a schematic diagram of the proposed SIS (superconducting-insulating- 


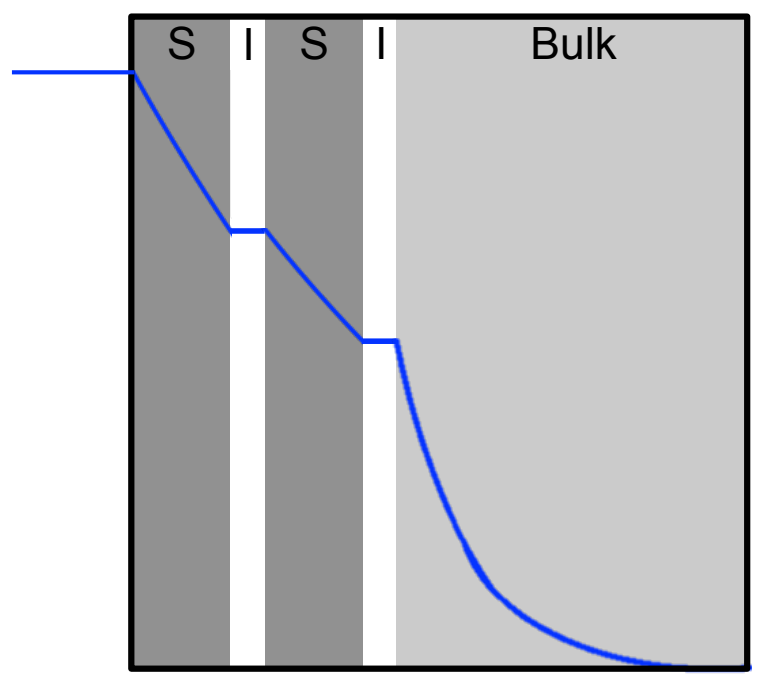

FIG. 1.5: Schematic Illustration of proposed SIS superstructures showing the attenuation of the induced radio frequency magnetic field as it passes through successive SIS superstructures screening a bulk superconductor.

TABLE 1.1: Comparison of candidate superconducting materials. [6]

\begin{tabular}{cccccc}
\hline \hline Material & $\begin{array}{c}\lambda(0) \\
\mathrm{nm}\end{array}$ & $\begin{array}{c}\xi(0) \\
\mathrm{nm}\end{array}$ & $\begin{array}{c}B_{s h} \\
\mathrm{mT}\end{array}$ & $\begin{array}{c}T_{C} \\
\mathrm{~K}\end{array}$ & $\begin{array}{c}\rho_{n} \\
\mu \Omega \mathrm{cm}\end{array}$ \\
\hline $\mathrm{Nb}$ & 50 & 22 & 210 & 9.2 & 2 \\
\hline $\mathrm{Nb}_{3} \mathrm{Sn}$ & 111 & 4.2 & 410 & 18 & 8 \\
\hline $\mathrm{MgB}_{2}$ & 185 & 4.9 & 210 & 40 & 0.1 \\
\hline $\mathrm{NbN}$ & 375 & 2.9 & 160 & 16 & 144 \\
\hline \hline
\end{tabular}


superconducting) multilayer structure. Here, candidate thin film superconductors with more desirable properties $\left(\mathrm{MgB}_{2}\right.$ or $\mathrm{NbN}$ for example) are intercalated with very thin insulating layers ( $\approx 2 \mathrm{~nm}$ of $\mathrm{MgO}$ for example) which provide shielding for a bulk superconducting material such as $\mathrm{Nb}$. This scheme is reliant upon thin superconducting layers having a coating thickness $d$ less than the $\lambda_{L}$ (London penetration depth) to take advantage of a thin film effect where the material will remain in the Meisnner state at applied parallel magnetic fields much higher than bulk critical field values.

\subsection{Scope of Dissertation}

The primary focus of this dissertation is on fundamental structure-property correlations in systems of superconducting thin films grown epitaxially on ceramic substrates, specifically $\mathrm{Nb} / \mathrm{MgO}, \mathrm{Nb} / \mathrm{Al}_{2} \mathrm{O}_{3}$, and $\mathrm{MgB}_{2} / \mathrm{Al}_{2} \mathrm{O}_{3}$. The early chapters introduce relevant ideas of superconductivity and a survey of concepts related to the growth and surface evolution of thin films. Chapter 4 gives an overview of the experimental tools used to generate and characterize the samples. Chapter 5 summarizes a series of related experiments, the results of which have already been published in peer-review journals $[7,8,9,10]$. Readers interested in closely-related work with $\mathrm{NbN}$ and SIS multilayer structures may refer to W. Roach's thesis "Superconducting Thin Films for SRF Cavity Applications: A Route to Higher Field Gradient Linacs" for further treatment [11]. I 


\section{CHAPTER 2}

\section{Superconductivity}

Salient macroscopic characteristics of superconducting materials include the observation of lossless electrical conductivity in DC applications and the spontaneous expulsion of applied magnetic fields from the superconducting bulk below a material-dependent critical temperature $T_{C}$ (Meissner-Ochsenfeld effect). Early measurements to establish the DC resistivity of persistent superconducting loops have merely placed lower bounds on the half-life of such systems on the order of 100,000 years [12].

The phenomenon of superconductivity was first observed by Dutch physicist Heike Kamerlingh Onnes in 1911. A prelude to this 1913 Nobel Prize winning discovery and auspicious milestone in cryogenic technology, Onnes was also the first to successfully utilize a liquefaction process for the production of liquid helium. ${ }^{1}$ His observations helped resolve lingering questions about the behavior of conducting materials near absolute zero. Many scientists of the age, including Lord Kelvin, anticipated that at extreme low temperatures

\footnotetext{
${ }^{1}$ The study of low temperature phenomena requires access to cryogens and the practical study of superconductors is reliant upon the availability of cryogenic fluid technology. With the growth of superconducting technology in medicine, science, and industry there is growing concern over the cost and availability of liquid helium. Unfortunately, helium is a nonrenewable resource and as such, improved recapturing systems and alternative refrigeration techniques are being researched (i.e. using other cryogenic materials like hydrogen).
} 
kinetics would dictate that conduction electrons would slow or come to a complete stop at or near absolute zero and, therefore, resistivity ought to approach infinity. However, while measuring the DC electrical resistivity of solid Hg, Onnes noticed a sharp decline in resistivity (essentially to zero) when the mercury was cooled near $L_{H e}$ temperatures $\sim 4.2$ K; see Fig 2.1.

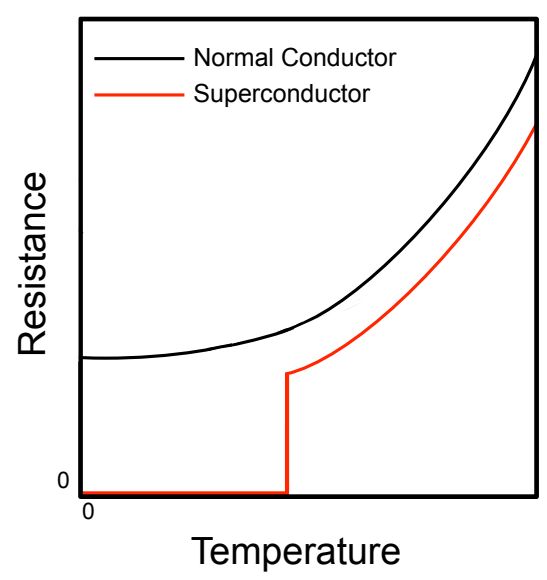

FIG. 2.1: Resistivity as a function of temperature for conventional and superconducting materials.

More than a century later, superconducting materials and applications thereof remain at the center of intense scientific and industrial research. The list of known superconducting materials continues to grow even as innovative uses for superconducting properties are exploited in many devices. In the field of medicine, large superconducting electromagnets are used in magnetic resonance imaging (MRI) or nuclear magnetic resonance (NMR) machines in hospitals to create strong, uniform, large-volume magnetic fields for medical imaging and diagnosis. Superconducting magnets can also be used to create and shape strong magnetic fields for plasma confinement. Superconductors are extremely sensitive to externally applied magnetic fields, thus making them ideal for sensing applications such 
as the SQUID magnetometer discussed in Section 4.3.

\subsection{Meissner effect}

The Meissner effect is often described as the spontaneous expulsion of an externally applied magnetic field from a superconductor as it transitions from a normal state into the superconducting state. This relationship between superconductivity and magnetic fields was first discovered by Walther Meissner and Robert Ochsenfeld in 1933. Their careful measurements of the magnetic field proximal to the surface of superconducting $\mathrm{Pb}$ and Sn directly demonstrated that superconductors are not merely "perfect" conductors and that this distinctive diamagnetic response - strong diamagnetism or Meissner effect - is a defining trait of superconductors.

Conceptually, a superconductor in the presence of an externally-applied magnetic field responds by establishing surface currents which induce a $\mathbf{B}$ field that directly and exactly opposes the applied field. Moreover, if a conducting material undergoes a superconducting transition in the presence of an applied field, it will spontaneously expel the magnetic field from the bulk. This response can be seen for applied field values up to a critical field value $H_{C 1}$, above which superconductivity is broken and the material transitions to a non-superconducting state.

The spontaneous exclusion of the magnetic field from the superconducting bulk cannot be fully explained by classical electrodynamics. It is useful to contrast the features of an idealized perfect electric conductor with that of a superconductor. A perfect conductor is one that will not suffer dissipative resistive losses from a steady current and will continue to flow unimpeded until acted on by an external force - a trait shared by superconductors. Additionally, perfect conductors require a constant magnetic flux such that any applied field will not effect a change in the conductor's internal field configura- 

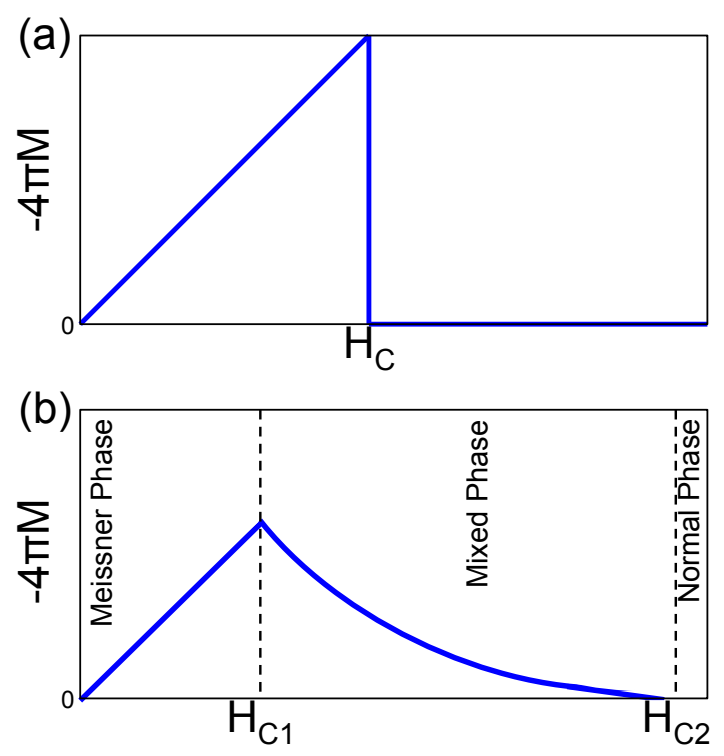

FIG. 2.2: Dielectric response of superconductors (a) Type-I and (b) Type-II superconductors.

tion. Here the observation of the Meissner effect and the quantization of magnetic flux in superconductors help fingerprint superconductors and distinguish them from an idealized "perfect" conductor.

There exist many classification schemes for types of superconductors such as those distinguished by material type (alloys, ceramics, pure elements, etc.) or by whether or not the material is understood within the context of BCS theory (so-called conventional or unconventional superconductors). One practical taxonomy has to do with the early observation of the magnetic response of the superconductors in the presence of an applied field (Figure 2.2). Type-I superconductors remain in the Meissner state until a critical field $H_{C 1}$ is reached, above which the material sharply returns to a normal state of conduction. Type-II superconductors are characterized by two critical fields - a lower critical field $H_{C 1}$ and an upper critical field $H_{C 2}$. A Type-II superconductor below $H_{C 1}$ is in a Meissner 

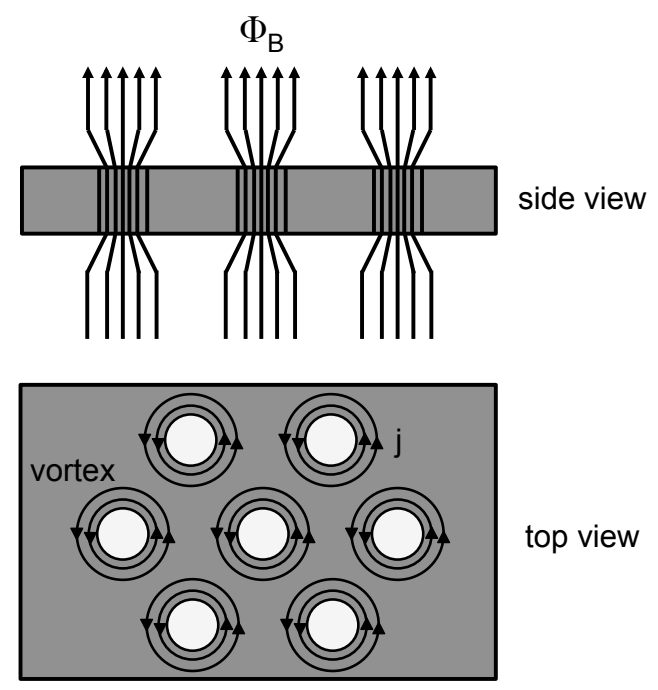

FIG. 2.3: Representation of magnetic vortices in a Type-II superconductor. Fluxons, absent pinning, typically form in a close packed hexagonal arrangement across the surface of the superconductor. The amount of flux enclosed by each fluxon is equal to $\Phi_{0}$.

phase, a thermodynamically reversible region where the Meissner effect expels the field from the bulk. In the region where $H_{C 1}<H<H_{C 2}$, the superconductor is in a mixed phase where magnetic flux penetrates the superconducting bulk in the form of quantized flux tubules know as fluxons or magnetic vortices (such as those represented in Figure 2.3). Some materials, like $\mathrm{MgB}_{2}$, have demonstrated $H_{C 2}>10 \mathrm{~T}$, remaining in the mixed superconducting state at impressive applied magnetic fields.

\subsection{London Theory}

An early phenomenological description of superconductivity was developed by brothers Fritz London and Heinz London. Their eponymous London equations accurately capture the more tangible macroscopic characteristics of superconductors - namely, zero DC resistivity and the Meissner Effect. This description begins by treating the supercon- 
ducting charge carriers as free charges experiencing uniform Lorentz forces arising from externally applied $\mathbf{E}$ and $\mathbf{B}$ fields and relating the current density of superconducting charge carriers $j_{s}$ to these fields. The London equations expressed in terms of measured fields are

$$
\frac{\partial \mathbf{j}_{\mathbf{s}}}{\partial t}=\frac{n_{s} e^{2}}{m_{e}} \mathbf{E}
$$

and

$$
\nabla \times \mathbf{j}_{\mathbf{s}}+\frac{n_{s} e^{2}}{m_{e}} \mathbf{B}=0
$$

where $n_{s}$ is the density of superconducting charges, and $e$ and $m_{e}$ are the charge and mass of the electron, respectively. The expression in (2.1) is an expression of infinite conductivity in an ideal conductor. Equation (2.2) represents the phenomenological leap that accounts for the Meissner effect — the establishment of a surface current in such a way to oppose an applied magnetic field. Whereas the magnetic field and current density in a so-called perfect conductor must satisfy

$$
\frac{\partial}{\partial t}\left(\nabla \times \mathbf{j}_{\mathbf{s}}+\frac{n_{s} e^{2}}{m} \mathbf{B}\right)=0
$$

the requirement that the magnetic field inside a superconductor be precisely zero (rather than simply time independent) necessitates that the parenthetical expression in (2.3) be strictly equal to zero as well. Applying Ampère's law

$$
\nabla \times \mathbf{B}=\mu_{0} \mathbf{j}_{\mathbf{s}}
$$


to (2.2) gives an expression for the magnetic field inside of a superconductor

$$
\nabla^{2} \mathbf{B}=\frac{1}{\lambda_{L}^{2}} \mathbf{B} \quad \lambda_{L} \equiv \sqrt{\frac{m}{\mu_{0} n_{s} e^{2}}},
$$

where $\lambda_{L}$ is the so-called London penetration depth. ${ }^{2}$ Similarly, an expression for the current density of superconducting charge carriers is obtained

$$
\nabla^{2} \mathbf{j}_{\mathbf{s}}=\frac{1}{\lambda_{L}^{2}} \mathbf{j}_{\mathbf{s}}
$$

For a simple geometry where the boundary between free space and a superconducting sheet is normal to the $x$-axis, with the superconductor extending into the positive $x$ direction. and magnetic field $\mathbf{B}$ pointing in the $z$-direction, the magnetic field and charge density inside the superconductor can be expressed as

$$
B_{z}=B_{0} e^{-\frac{x}{\lambda_{L}}} \quad j_{y}=-\frac{1}{\lambda_{L}} B_{0} e^{-\frac{x}{\lambda_{L}}}
$$

From here, a physical interpretation of $\lambda_{L}$ defines a characteristic length scale through which the magnetic field is attenuated before disappearing into the bulk, thus the effectiveness of the shielding super currents is not perfect and the presence of the supercurrent is confined to the topmost layers of the superconductor.

\footnotetext{
${ }^{2}$ Foreshadowing BCS theory and the idea that superconducting charge carriers are actually a bound state of two electrons, we note here that the London penetration depth remains invariant under the replacement $n_{s} \rightarrow n_{c}=n_{s} / 2, e \rightarrow 2 e$ and $m_{e} \rightarrow m_{c}=2 m_{e}$.
} 


\subsection{BCS in brief}

A quantum mechanical justification of the London equation remained opaque until 1957 when Bardeen, Cooper, and Schrieffer put forth a microscopic theory of superconductivity (BCS theory) which supposed that the superconducting charge carriers were electrons of opposite spin forming a bound state - a bosonic quasi-particle dubbed a Cooper pair. This pairing is mediated by a phonon interaction as the electrons travel through a crystalline lattice. Essentially, the picture is that electrons traveling through the lattice distort the surrounding ions. This lattice distortion in turn produces a net Coulombic attraction that interacts with another electron (forming a pair). That phonons mediate this process is supported by the "isotope" effect [13].

When the superconductor condenses into the superconducting state, all of the bosonic Cooper pairs occupy a single quantum state. With the formation of the superconducting condensate, a temperature-dependent energy gap $\Delta$ also opens in the electronic structure. At $T=0 \mathrm{~K}$, the critical temperature is related to the energy gap $\Delta$ by

$$
1.76 k_{B} T_{C}=\Delta(0)
$$

This energy gap opens up about the Fermi energy as the density of states changes as electrons pair off into Cooper pairs.

The quantum origins of superconductivity are also supported by the observation that the amount of magnetic flux through superconducting loops is found to be quantized in units of

$$
\Phi_{0}=\frac{h}{2 e}=2.0678 \times 10^{-15} \mathrm{~Wb}
$$


Because of the intimate relationship between magnetism and superconductivity, superconducting materials have been exploited to take advantage of quantum interference phenomena. Of enormous practical import, Josephson junctions have been utilized in many devices including very precise magnetometers, like the superconducting quantum interference device (SQUID) discussed in Section 4.3.

Because electronic interactions are typically the dominant mechanism for thermal transport in materials, the condensation of electrons into Cooper pairs also has ramifications for the thermal conductivity of superconductors. This takes on extra importance in the area of SRF cavities, which must be able to slough off excess accumulated heat during the microwave cycles. To this end, the ideal scenario is to utilize a bulk cavity material with excellent thermal transport properties, such as copper $(\mathrm{Cu})$, and then coat this cavity with superconducting thin films.

Ginzburg-Landau theory (applied in many areas of physics) distinguishes between the two types of superconductors based upon the ratio of two characteristic superconducting lengths. The unitless Ginzburg-Landau parameter $\kappa$ is

$$
\kappa=\frac{\lambda_{L}}{\xi}
$$

where $\lambda_{L}$ is the London penetration depth and $\xi$ is the coherence length, a characteristic length scale over which Cooper pairs are correlated.

Type-I superconductors are typified by $\kappa<1 / \sqrt{2}$ and Type-II by $\kappa>1 / \sqrt{2}$. Note that $\xi$ is proportional to the mean free path of the conduction electrons in the metal. Most alloys have a shorter mean free path when compared to pure elements, thus are more likely to be Type-II superconductors. As mentioned previously, Type-II superconductors allow flux penetration in the form of magnetic vortices, or fluxons, each containing $\Phi_{0}$, surrounded by a Cooper-pair current. Changing the applied field changes the density of 
coverage (each fluxon is a normal conducting region with an area $\sim \pi \xi^{2}$ ), thus we can envision the upper critical field $H_{C 2}$ to be the field value where these vortices begin to overlap. The upper critical field is predicted by

$$
B_{C 2}=\frac{\Phi_{0}}{2 \pi \xi^{2}}
$$

In high $T_{C}$ superconductors, the coherence length is typically shorter than the grain boundaries of the superconductor, thus current flow from one grain to the next is strongly impeded.

For SRF applications, control of the microstructure of superconducting films is critical. For microwave operation, the vortices that form in Type-II superconductors can be problematic. Magnetic vortices experience a Lorentz force when a changing magnetic field is applied. The viscous motion of the vortices about the superconductor can result in further dissipative losses, i.e. heating or flux flow resistance. This problem can be mitigated to a certain extent by "pinning" or fixing these vortices to a particular location in the crystalline lattice by the deliberate addition lattice defects or impurities.

Flux pinning in superconductors may have drawbacks depending on the application, as this pinning can result in a strong hysteresis response, thus the flux can be frozen into the superconductor even if the externally applied magnetic field is reduced to 0 . Consequently, above $H_{C 1}$ (the mixed state) the Type-II superconductor may not return to the pure Meissner state without first returning the superconductor to a normal state. This has implications for measurements of $H_{C 1}$, which is discussed further in section 4.3. 


\subsection{Two-Fluid Model and Surface Resistance of Su- perconductors}

The charge carriers in a superconductor can be thought of consisting of two distinct species or fluids — the condensate comprised of Cooper pairs and unpaired, normal-state conduction electrons. The normal-state electrons in the presence of an electric field obey Ohm's law

$$
J_{n}=\sigma E_{0} e^{i \omega t}
$$

The infinitely-conducting Cooper pairs receive an acceleration and current

$$
m \dot{v}=-2 e E_{0} e^{i w t} \quad J_{s}=-i \frac{n_{c} 2 e^{2}}{m_{e} \omega} E_{0} e^{i \omega t}
$$

Combining the two fluids together yields the total current density

$$
J=J_{n}+J_{s}=\sigma E_{0} e^{i \omega t}
$$

with a complex conductivity

$$
\sigma=\sigma_{n}-i \sigma_{s}, \quad \text { where } \quad \sigma_{s}=\frac{2 n_{c} e^{2}}{m_{e} \omega}=\frac{1}{\mu_{0} \lambda_{L}^{2} \omega}
$$


The surface resistance is the real part of the complex impedance

$$
R_{\text {surf }}=\operatorname{Re}\left(\frac{1}{\lambda_{L}\left(\sigma_{n}-i \sigma_{s}\right)}\right)=\frac{1}{\lambda_{L}} \frac{\sigma_{n}}{\sigma_{n}^{2}+\sigma_{s}^{2}}
$$

at microwave frequencies $\sigma_{n}^{2} \ll \sigma_{s}^{2}$, thus the above expression may be approximated $R_{\text {surf }} \propto \sigma_{n} /\left(\lambda_{L} \sigma_{s}^{2}\right)$. Note the less-than-intuitive result that the surface resistance is proportional to the normal state conductivity.

Applying the Drude expression for conductivity we arrive at an expression for the BCS surface resistance

$$
R_{B C S} \propto \lambda_{L}^{3} \omega^{2} l e^{\left(-1.76 T_{c} / T\right)},
$$

where $l$ is the mean free path of conduction electrons. The BCS surface resistence depends exponentially on temperature and is proportional to the square of the driving frequency.

\subsection{Thin Film Superconductors}

In very thin sheets of superconductor (i.e. where film thickness $d<\lambda_{L}$ ), the magnetic field is not completely expelled from the superconducting material; hence, less magnetic energy is expelled as compared to a bulk superconductor. This naively suggests that thin film superconductors may exhibit higher than bulk values for $H_{C 1}$. Indeed, theoretical and experimental work indicates a strong dependance of the critical supercurrent $J_{C}$ and $H_{C 1}$ on film thickness and relative orientation within an applied field [14]. For superconducting films whose thickness, $d$, is less than the London penetration depth, $\lambda_{L}$, the in-plane lower critical field is 


$$
B_{C 1}=\frac{2 \Phi_{0}}{\pi d^{2}} \ln \frac{d}{(1.07) \xi}, \quad d<\lambda_{L}
$$

where $\Phi_{0}$ is the flux quantum and $\xi$ is the coherence length [5]. This critical field enhancement is extremely sensitive to alignment of the film surface and is maximal when the applied field is parallel to the thin film surface. The geometric conditions for this enhancement coincide with the field geometry of SRF cavities. 


\section{CHAPTER 3}

\section{Thin Film Growth and Scaling in Dynamic Systems}

This chapter is devoted to a conceptual survey of relevant topics in the dynamics of thin film growth. The dynamics of thin film growth leading to different microstructure and surface morphology is one of the important factors in understanding the microscopic processes associated with a growth mechanism leading to well-tailored materials with desirable properties. Often, the performance of nanodevices is reliant upon the minimization of surface and interfacial roughness, whereas for other applications, such as materials designed for improved adhesion or for catalytic processes, rough interfaces are sought $[15,16]$. As emphasized previously, in SRF applications the relevant properties are inherently a surface phenomenon because of the shallow penetration depth of the RF fields in superconductors [17]. The crystalline structure, particularly crystalline defects — atomic defects, impurities, extended defects, and grain boundaries - influence the material mechanical and superconducting properties. Additionally, the surface morphology of superconducting thin films in SRF applications can also contribute local field enhancements, which effectively 
lower the maximum sustainable critical field and allow early fluxoid entry and increased dissipation, which adversely affect the SRF performance of real surfaces with respect to idealized ones. An understanding of the dynamics of thin film growth and characterizing set of growth conditions leading to improved structural and morphological traits is an important step towards realizing SIS multilayers.

\subsection{Nucleation and Growth of Thin Films}

It is useful to first establish a working definition for a thin film. A superficial definition of a thin film might be any structure where one spatial dimension has has been constrained with respect to other spatial dimensions, e.g. a coat of paint on a living room wall $\sim 2 \mu \mathrm{m}$ thick; however, a more refined definition considers the scale of the constituent components and might be narrowed to describe any structure whose properties are dominated by the energetics at the surface, rather than in the bulk material. Hence thin films, with their constrained geometries, can exhibit distinct material properties as compared to their bulk counterparts.

It is also useful to consider an atomistic point of view when considering thin film nucleation and growth. The first thing to note is that free surfaces are generally energetically distinct from bulk. One way to intuit this is to consider the bonding between constituent species; that is to say, atoms at free surfaces have fewer neighboring interactions when compared to those in the bulk and are thus are less constrained. As a result some materials can undergo a surface reconstruction, a structural reconfiguration giving rise to periodic surface superstructures distinct from the underlying bulk order, in an effort to minimize the Gibbs free energy of the system [18].

In thin film deposition, adatoms interact energetically with a potential defined by the substrate crystal field. Epitaxial thin films are quasi single-crystal films which are 
coherently oriented with respect to a crystalline substrate. Epitaxial growth is further divided into two types: homoepitaxy where film and substrate are the same material and heteroepitaxy where film and substrate are different materials. Such thin films may closely mimic the underlying substrate structure, deviate drastically or exhibit exotic unstable phases maintained by strain mechanisms; regardless, there is a fixed, relative structural, geometric and structural relationship — a registry — between substrate and film.

As a heuristic rule, there typically needs to be a good match (with $10 \%$ between the lattice parameters of a thin film and substrate in order to achieve epitaxy. As the bulk lattice parameters rarely coincide between disparate materials, epitaxial films usually demonstrate some degree of stress and strain, which is typically relieved within the film with increasing distance from the substrate surface by dislocation or inclusion mechanisms. Structure is intimately tied with physical property (particularly in the case of superconductors); thus, thin films provide a versatile platform for manipulating material properties by subtle manipulation of thin film structure and morphology, leading to novel properties and improved performance.

If we imagine a pristine crystalline lattice, serving as a substrate, exposed to an incoming flux of gas particles, there is some probability that the particles stick to the surface and a corresponding probability of reflection, whose relative rates determine an overall growth rate. Thus, the thin film deposition process proceeds by the adsorption of impinging molecules or atoms either by chemisorption, where the chemical nature of the adsorbate changes by covalent or ionic bonding with the substrate constituents or by physisorption, a van der Waals bonding with the substrate [18].

The manner in which the structure and morphology evolve is system dependent. As a practical matter, substrates are rarely atomically flat and may have additional structural features such as step edges, terraces, corners, defects and vacancies, each of which modify the energetics of the system on a local scale. Experimentally, in thin film deposition, 
it is possible to provide additional energy via substrate heating or by kinetic deposition methods to overcome energetic diffusion barriers and thus encourage diffusion and surface mobility of adatoms, enabling them to seek more energetically favorable configurations.

The structure zone diagram (SZD) presented by Thornton describes the interplay between thermal and kinetic energy driving film growth and the resulting characteristics and texture of the grown film [19]. The SZD has recently been extended by A. Anders to include additional novel deposition techniques that promote surface diffusion, resulting in more dense films (Figure 3.1) [20]. He observes an interesting parameter space where the particle's kinetic energy is between the surface displacement energy and bulk displacement energy which may prompt epitaxial growth at lower temperatures as compared with less energetic deposition scenarios.

Thin film growth can be thought to occur in stages: 1) nucleation 2) film growth and 3) coalescence. The main processes responsible for a particular surface morphology during thin film deposition are (i) direct addition of atoms, (ii) removal of atoms, and (iii) diffusive movement of atoms. All these processes can be present during a particular thin film growth experiment. Random deposition and ballistic deposition with relaxation are two of the models that are used to understand the formation of thin films. In the latter case, the adatom approaches the surface in columnar fashion and adheres to the substrate. If it also preferentially locates adjacent to a nearest neighbor, this may yield a correlated and self-affine interface [21]. Other material issues must be considered when characterizing epitaxial thin film growth, since nucleation and relaxation kinetics on different surfaces may give rise to film properties that deviate from those in bulk materials. Some relevant considerations include limited material supply as well as stress contributions due to lattice mismatch, which can induce significant surface roughness.

The deposition parameters — such as growth rate, the substrate temperature, sputtering pressure, film thickness, and ion-to-atom ratio — affecting thin film growth can 


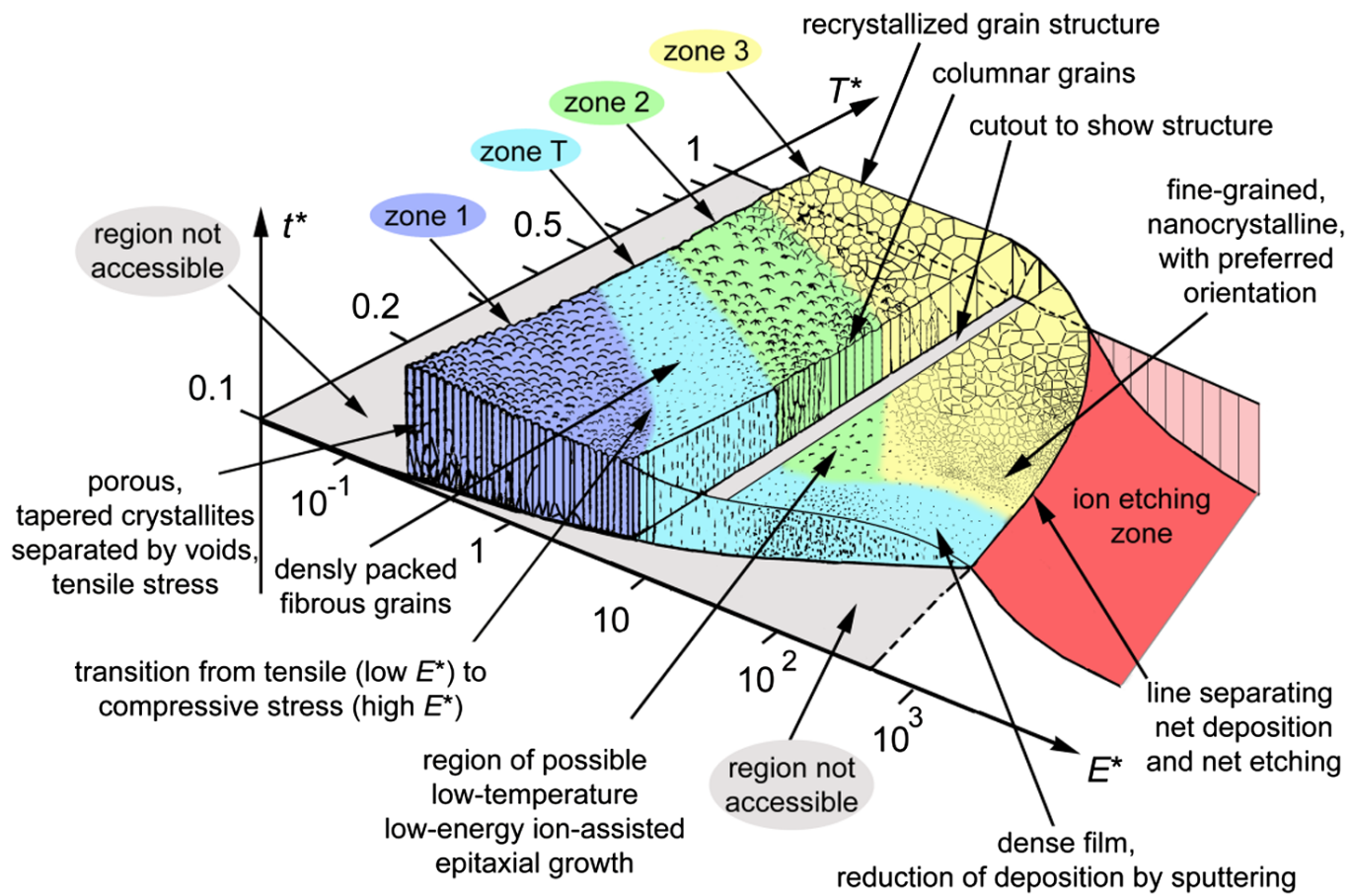

FIG. 3.1: Modified SZD diagram (reproduced from [20] with permission from the author and Elsevier Publishers). Diagram illustrates how thin-film grain structure is expected to vary with film thickness $t^{*}$, growth temperature $T^{*}$, and deposition energy $E^{*}$. 


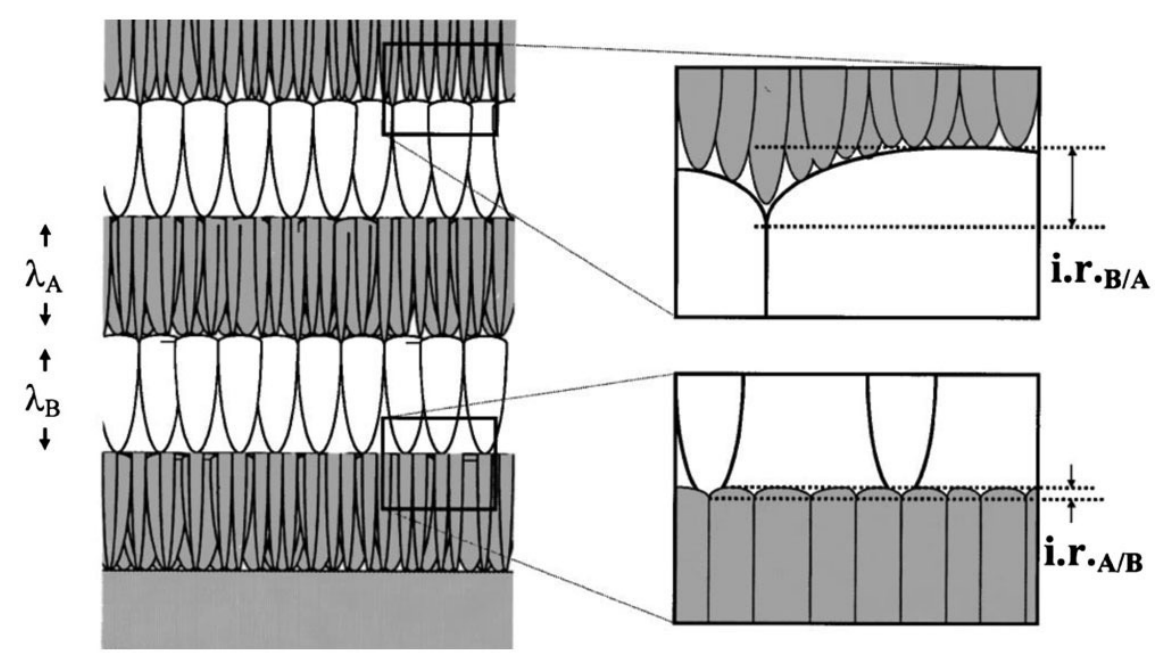

FIG. 3.2: Cross-sectional illustration of a hypothetical multilayer coating formed with equal thicknesses $\lambda \mathrm{A}$ and $\lambda \mathrm{B}$ demonstrating a difference of nucleation density between materials $\mathrm{A}$ and $\mathrm{B}$ in such a way as to control surface roughness via these competing mechanisms. The magnified images illustrate the relative interfacial roughness associated with each material. Reproduced from [22] with permission from the American Institute of Physics. 
strongly influence surface morphology and microstructure. Adequate control of these factors is valuable, particularly for the nucleation of the first sublayer upon the substrate and is of paramount importance when attempting a multilayered coating (see Figure 3.2) since the growth mechanism peculiar to each sputtered material can be strongly modified since roughness and topography will continue to evolve with subsequent deposition of each sublayer [22].

When depositing epitaxial thin films, nucleation and growth kinetics can lead to different growth modes ranging from layer-by-layer growth (i.e., Frank-van der Merwe) to island formation, or three-dimensional (i.e. Volmer-Weber) growth modes [23, 24]. Physical process that can lead to the latter growth mode include step-edge diffusion barriers and stain relaxation mechanisms due to mismatch between film and substrate lattice parameters $[22,25]$. Additionally, the evolution of the surface during epitaxial growth can lead to faceting, further hindering the possibility of smooth surfaces. Thus, the growth mode during the nucleation stage can affect the ultimate surface morphology and exhibit fractal-like characteristics whose self-similarity, i.e. self-affinity, persists throughout the temporal and spatial surface evolution during subsequent growth and has a profound effect on relevant physical properties.

\subsection{Fractals, Self-similarity and Scaling}

In the 1970s, Benoit Mandelbrot codified some of the earliest formal, geometric descriptions of a class of objects (mathematical and physical) which he termed fractals. Broadly speaking, an object with "fractal" characteristics can be thought of as one that retains elements of self-similarity or self-symmetry when considered over a range length scales. Following Mandelbrot's mathematical formalism, a flurry of activity across a broad range of disciplines, particularly within the physical sciences, sought to describe and un- 
derstand a vast range of complex natural systems within this formalism [26].

With the rise of computational physics throughout the 1980s and 1990s, it became ever increasingly viable to carry out large-scale simulations utilizing Monte Carlo methods to better model and simulate pattern formation in complex, dynamic systems. For example, early successful fractal descriptions came from Witten and Sander's model of diffusion limited aggregation (DLA) for systems whose dominant mechanism for mass transport is diffusion - of particular relevance to thin film systems [27]. Fractal concepts and attending scaling ideas provide connections between seemingly unrelated systems including (but not exhaustively so) turbulent flow, Brownian motion, percolation, polymer networks, galaxy formation, erosion processes and thin film growth. Topics such as selforganization, branching behavior and critical phenomena can be broadly understood and described within this framework [28].

It is important to distinguish between mathematical and "real" fractals. Mathematical fractals, like the canonical Koch Snowflake, can be likened to so-called pathological functions such as the Weierstrass function - the first known example of a real-valued function that is everywhere continuous and differentiable nowhere. The Koch snowflake, here constructed iteratively in Figure 3.3, is an example of a fractal object that has a finite area with a perimeter length approaching infinity [26].

In "real" or finite physical systems, the idea of self-similar or self-affine patterns takes on a statistical interpretation. For example, in the context of solid state systems, the constituent arrangement of atoms within the material define a minimum length scale or cutoff at interatomic distances, below which the concept of self-correlation and selfsimilarity loses meaning. Similarly, there is a characteristic saturation length scale above which self-similarity breaks down, defining an upper cutoff length scale. Thin film growth is a stochastic process, thus identically prepared systems may not evolve in a microscopically identical fashion; however, under similar thermodynamic conditions, separately prepared 

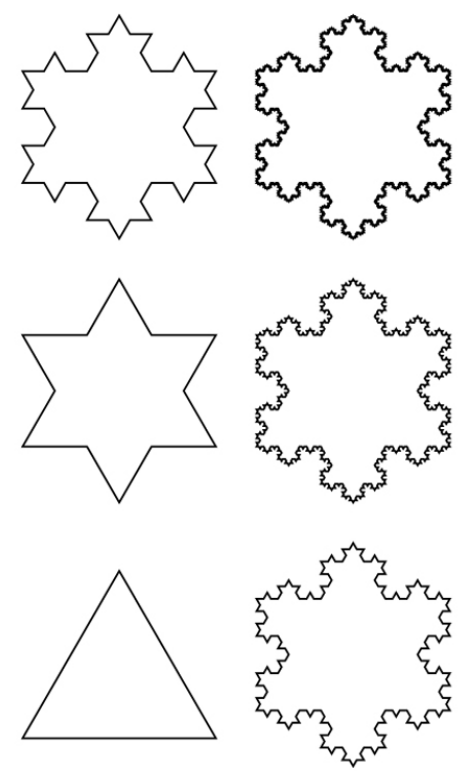

FIG. 3.3: Iterative construction of a Koch snowflake as an example of a mathematical fractal.

systems may have a statistical correlation over certain length scales. Thus, the observation is that for many physical systems, including thin film systems, the emergent long range order arises from noise and the short range microscopic forces driving the non-equilibrium process.

\subsubsection{Family-Vicsek Scaling}

The Family-Vicsek scaling ansatz presupposes that surfaces arising from non-equilibrium processes can be described by a scale-invariant form in both space and time and can be fully characterized by a finite set of scaling parameters. It is assumed that stochastic processes drive the surface evolution and that the resulting features will have a self-affine form $[29,28]$. In this scenario, the dynamic evolution of the roughness of the system in question can be fully described by a pair of scaling exponents, $\alpha$ and $\beta$, the global roughness exponent and growth exponent, respectively. These experimentally determined 
parameters, when compared with those calculated from growth models, will implicate particular, responsible growth mechanisms and serve to classify these processes within a scaling universality class.

Here, it is assumed that the function which correlates characteristic lengths will assume a power-law form and that the Family-Vicsek dynamic scaling ansatz

$$
w(L, t)=t^{\beta} f(L \xi(t))
$$

will hold. The function $f(u)$ takes the form

$$
f(u) \sim\left\{\begin{array}{ll}
u^{\alpha} & \text { if } u \ll 1 \\
\text { constant } & \text { if } u \gg 1
\end{array},\right.
$$

where the surface interface width $w$ is taken to be the RMS roughness of the surface averaged over the lateral length $L$ - the lateral dimension or scan size of the surface under consideration - after time $t$ (or equivalently thickness in the case of a constant growth rate), and has two distinct asymptotic regimes depending on the length scale examined. Here, $L_{c}$ (nominally taken to be morphological island size) defines a critical length scale over which the surface features are no longer correlated and scales as $t^{1 / z}$, where $z \equiv \alpha / \beta$ is the dynamic exponent.

\subsubsection{General Dynamic Scaling}

The self-affine approach to dynamical scaling has been successfully applied to a vast array of dynamic surfaces (such as the erosion of coastlines, chemical etching processes, and ballistic deposition); however, there are certain systems in which the scaling patterns demonstrate aberrant behavior between the global (long-range or saturated) and local 
(short) length scales [28]. In this case, a common set of scaling exponents is no longer adequate to simultaneously characterize the disparate global and local dynamic scaling behavior; hence, additional scaling parameters are necessary to fully classify surfaces typified by this so-called anomalous scaling.

General Dynamical Scaling applies a more generic, yet analogous, scaling ansatz, formulated in Fourier space, which introduces additional independent scaling parameters, extending the taxonomy and further classifies anomalous scaling into invariant subclasses [30]. Hence, General Dynamic Scaling can be thought of as an extension of Family-Vicsek scaling with the inclusion of an independent scaling parameter obtained from the spatial frequency space. Here, the scaling assumption is that the Power Spectral Density (PSD) function defines a structure factor $S$

$$
S(\mathbf{k}, t)=\langle H(\mathbf{k}, t) H(-\mathbf{k}, t)\rangle
$$

where $H$ is the Fourier transform of the surface height function, $\mathbf{k}$ is the spatial frequency, and $t$ is the time (or film thickness in our case as the growth rates of our films are reproducible and constant in sputtering deposition). Here the PSD assumes a general form (for a $2+1$ dimensional system) and the spectral exponent $\alpha_{s}$ quantitatively captures the anomalous scaling independent of local and global values for $\alpha$ and $\beta$. The value of the $\alpha_{s}$ is evaluated from the slope along the linear region of the $\log$-log plot $S$ vs. $k$ in the realm of large $k$, demarcates scaling behavior by

$$
S(k, t)=k^{-\left(2 \alpha_{s}+2\right)} t^{2\left(\alpha_{l o c}-\alpha_{s}\right) / z},
$$




$$
\alpha_{s}<1 \Rightarrow \alpha_{l o c}=\alpha_{s}\left\{\begin{array}{l}
\alpha_{s}=\alpha \Rightarrow \text { Family-Vicsek } \\
\alpha_{s} \neq \alpha \rightarrow \text { intrinsic } \\
\alpha_{s}=\alpha \Rightarrow \text { super-rough } \\
\alpha_{s} \neq \alpha \rightarrow \text { new class }
\end{array}\right.
$$

Some recent studies have led to the hypothesis that large scaling exponents (i.e. $\alpha>1$ ) and anomalous roughening of the growing surfaces are a consequence of non-local effects such as hindered diffusion and shadow instabilities [31]. A systematic study controlling parameters affecting the kinetics of the growth may implicate underlying growth mechanisms and illustrate the relative virtues for a given set of deposition parameters. 


\section{CHAPTER 4}

\section{Experimental Methods}

This chapter outlines the experimental methods and techniques used in the preparation and characterization of the thin film samples. The first section details the particular thin film fabrication techniques employed in the creation of the $\mathrm{Nb}$ and $\mathrm{MgB}_{2}$ samples referenced in this work. In particular, DC magnetron and reactive sputtering methods were used in the generation of $\mathrm{Nb}$ and $\mathrm{NbN}$ films and were carried out in Dr. R. A. Lukaszew's thin films lab at the College of William and Mary. $\mathrm{MgB}_{2}$ samples were generously provided by collaborators T. Tan and Dr. X. X. Xi from Temple University, prepared using a hybrid physical-chemical vapor deposition technique [9, 32, 33].

The remaining sections discuss the characterization tools and techniques used to assess the thin film samples. Section 2 outlines surface characterization (atomic force microscopy (AFM) and its role in characterizing the surface morphology) and structural characterization (reflection high-energy electron diffraction (RHEED) and X-ray diffraction (XRD)) used to quantify and qualify the microstructure of our samples. Section 3 provides an overview of magnetic characterization by a superconducting quantum interference device (SQUID), used in determining superconducting properties of the thin films. 


\subsection{Thin Film Deposition}

The ideal process of thin film growth is one where material is controllably transferred to, removed from, or rearranged on a surface, resulting in a functional material with desired properties. Material properties, particularly in the case of thin films, may be affected by surface morphology, interfacial roughness, microstructure (grain size, grain boundaries, impurities, etc.), material interfaces, stoichiometry, and film thickness. Often the material properties are correlated with and sensitive to their composition and structure, thus thin films offer a versatile platform for tailoring materials with specific properties for designer applications. Superconducting properties such as critical fields and the superconducting transition temperature are closely correlated with the microstructure of the material in thin films, thus control of the microstructure in thin film geometry provides an avenue for control over these properties.

A vast array of deposition methods exist and each may have advantages and disadvantages depending upon the application. These diverse methods are generally categorized as physical vapor deposition (PVD) or chemical vapor deposition (CVD). A PVD process is one where material is vaporized, generally from a solid source (as in sputtering) or a molten source (as in evaporation techniques) and then transferred, typically in a high vacuum (HV $\sim 10^{6}$ Torr) or ultra-high vacuum (UHV $\sim 10^{9}$ Torr) environment, to a substrate where the material subsequently condenses to form a film.

PVD systems are typically quite versatile, in that one particular system may be capable of producing a wide range of film samples without breaking vacuum. CVD processes typically involve a sequential flow of gaseous species along a substrate surface where a chemical reaction, sometimes with the aid of precursor chemicals, will occur and a film develops. These methods, exemplified by atomic layer deposition (ALD), are capable of producing single atomic or molecular layers, but may require the use of caustic or toxic 
reagents and gasses and thus call for advanced safety training and material disposal. CVD deposition reactors typically must be dedicated to the production of a particular material and may have additional safety requirements for operation.

No one deposition method is inherently better than any other. Each may carry advantages and disadvantages depending on the scale and type of material being developed. The choice of deposition method is often a balance of cost, availability, production volume, and acceptable tolerances in the quality. The needs of a laboratory producing proof of principle, coupon-sized samples will necessarily be different than the manufacturer producing films for industrial applications.

A wide range of high quality crystalline substrates are commercially available as templates for epitaxial growth, and to this end the choice and quality of substrate is a critical parameter, as films can be extremely sensitive to initial substrate conditions. For example, while preparing a series of $\mathrm{Nb}$ films grown on $\mathrm{MgO}(001)$, it was observed that under nominally identical growth conditions, the $\mathrm{Nb}$ films manifested in two distinct epitaxial registries. After eliminating possible sources of systematic error, ensuring that the identically-prepared substrates were secured from the same batch number, and fixing all controllable film deposition parameters, we concluded that the microstructure of individual substrates stochastically determined the epitaxial registry of the grown film. Minute differences in the substrate, such as miscuts or surface contaminants can drastically affect the nucleation and growth of thin films. A purportedly flat substrate, shiny to the eye, may actually contain miscuts or other surface irregularities. Indeed, controlled miscuts can be used to relieve strain and facilitate lattice matching for some epitaxial experiments. Substrates may also react with their environment over a long period of time. $\mathrm{MgO}$ in particular is known to react in air and its quality may degrade over time, requiring long-term storage in desiccation containers.

Thin film quality and properties may vary depending upon initial surface conditions 
and by the manner in which they are produced and processed; thus the energetics and thermodynamics of thin film growth and the substrate condition determine the final structure. For a given growth method, there may be many ways to influence critical growth parameters. For instance, heating a substrate can increase thermal energy, allowing for easier mass transport of adsorbed species and increased diffusion rates. With many techniques, the kinetic energy of the deposited material may be controlled as well, providing more control in determining the ultimate structure and morphology.

\subsubsection{Vacuum Technology and Thin Films}

Access to HV and UHV conditions is a necessary prerequisite for many thin film deposition techniques. Contamination during growth is of primary concern and vacuum environments provide a means of minimizing contamination during film growth; however, even in "clean" UHV environments, there exists a background pressure of ambient contaminants which gives a net contaminant flux upon an otherwise pristine substrate surface. Here a competition between growth rates and contamination rates determines the rate at which impurities are produced during thin film growth. As the properties of thin films (typically less dense than bulk due to lattice defects and vacancies) may be sensitive to contaminants, this is of particular concern for SRF surfaces, as previously noted.

For magnetron sputtering, discussed further in the following section, the introduction of a working gas into the system places requirements on the vacuum system including impedance controls and pumps with adequate pumping speeds (e.g. cryogenic pumps or turbo molecular pumps) to maintain and stabilize the differential pressures required for operation. Two UHV systems were used to create the sputtered thin films discussed in this dissertation ( $\mathrm{Nb}, \mathrm{NbN}$, and $\mathrm{MgO})$ : (1) a modified Perkin-Elmer molecular beam epitaxy (MBE) system with 5 source ports outfitted with sputtering guns with additional cryogenic 
pumping and (2) a Veeco Bell Jar system with a turbo molecular pump.

The Perkin-Elmer — outfitted with a RHEED system, substrate heater and sample rotation system, as well as a residual gas analyzer — features a loadlock such that multiple samples can be introduced into the system without breaking vacuum. As such, the PerkinElmer system typically holds a base pressure on the order of $10^{-9}$ to $10^{-10}$ Torr. Most of the work with $\mathrm{Nb}$ was achieved in this system because $\mathrm{Nb}$ is a well known getter material and will rapidly oxidize in ambient atmospheric conditions, thus maintenance of the UHV environment allows for in situ RHEED characterization.

The Veeco system is primarily used for reactive sputtering processes, which include reactive gasses such as $\mathrm{O}_{2}$ or $\mathrm{N}_{2}$ along with the working gas (typically Ar). It has Viton ${ }^{\mathrm{TM}}$ seals whose vapor pressure limit the overall base pressure to around $10^{-6}$ Torr. Sample changes require bringing this system to atmospheric conditions, thus the system that was used primarily for reactive sputtering. The system is outfitted with a substrate heater designed to operate up to $600{ }^{\circ} \mathrm{C}$ in the presence of reactive gasses like oxygen.

\subsubsection{Magnetron Sputtering}

Many of the thin film samples discussed in this dissertation were fabricated using DC magnetron sputtering. This section is intended to give an overview of the sputtering mechanisms and necessary system requirements for operation, as well as the relative technical advantages and disadvantages of sputtering as compared with other deposition methods. Magnetron sputtering systems are commonly employed in both scientific and industrial contexts owing to the demonstrated versatility and adaptability of these techniques in producing high quality thin films for a wide range of materials and applications [18].

Sputtering is a PVD process whereby energetic particles impinge upon a target material, transferring kinetic energy to the target, thereby ejecting material to be deposited. 
The energetic ions in the sputtering process are generated in a glow discharge plasma. A working gas (usually a heavy noble gas, e.g. Ar) is ionized and the resulting ions are accelerated toward a target material serving as a cathode. The bombarding ions have an opportunity to be scattered, adsorbed, implanted (depending on the incident energy) and they may eject or "sputter" surface atoms from the target material.

In magnetron sputtering, magnets are configured to confine the plasma close to the target material, increasing sputtering efficiency and yield. Plasma confinement also minimizes sputtering in other areas of the deposition chamber, including the substrate. DC sputter deposition is a prevalent physical vapor deposition method for thin metallic films. A vacuum system operating in the HV regime $\left(10^{3}\right.$ to $10^{8}$ Torr $)$ is a prerequisite. For most physical deposition processes, a low system base pressure is generally desirable in order to minimize contamination during film deposition. As mentioned previously, even with sophisticated pumping methods, complete evacuation of deposition chambers is not feasible, thus even in the cleanest of vacuum environments there will be a residual flux of contaminants, rendering a nominally clean substrate "dirty" over time in a vacuum chamber.

Some surface modification techniques, such as ion beam assisted deposition (IBAD) and plasma etching, make use of impinging ions on the substrate or growing film. The incident ions impart energy to the surface, resulting in heating, surface modification and improved adatom mobility. Ion etching and plasma treatments of surfaces can result in significant changes to the surface microstructure and morphology of a film. A comprehensive review of modern magnetron sputtering configurations (like unbalanced magnetron sputtering) will not be addressed here, but can be found in the literature [34]. 


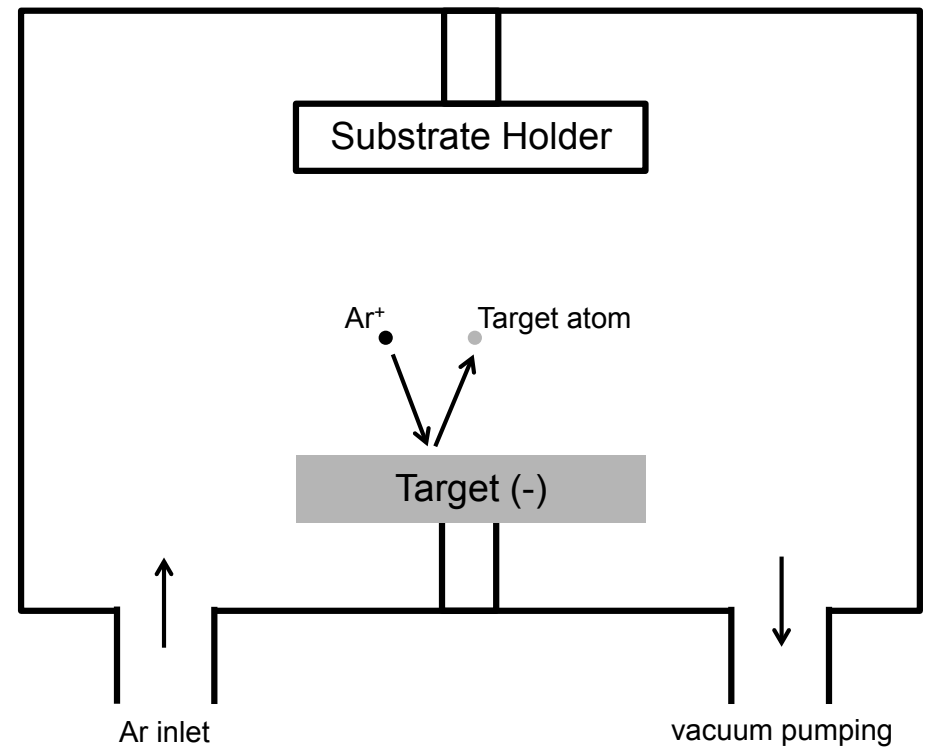

FIG. 4.1: Illustration of a magnetron sputtering system. 


\subsubsection{Pulsed Sputtering and Reactive Sputtering}

DC sputtering techniques are not directly translatable to dielectric materials, such as $\mathrm{MgO}$ and $\mathrm{NbN}$. As a positive charge accumulates on the dielectric target, electrostatic repulsion slows ion bombardment and, with a sufficiently large charge imbalance, a dielectric breakdown will occur resulting in arcing across the target. This arcing can eject, in a decidedly uncontrolled fashion, droplets of target material into the deposition chamber and onto the substrate. This is an undesirable operational mode [35].

$\mathrm{RF}$ and pulsed power supplies are designed to eliminate charge buildup by charge cycling asymmetric positive and negative polarities, enabling stable sputtering for insulating materials. The power supply applies a negative bias for the majority of the cycle to achieve sputtering, followed by a positive bias, where electrons are pumped to the target to neutralize charge buildup. Many commercial pulsed power supplies, like the Pinnacle pulsed power supply used in these experiments, allow control of frequency and duration of the reverse bias during the power cycle. Many units have built-in arc suppression features utilizing electric feedback sensitive to the build-up of arc currents that respond by either rapidly reducing the power to the target or by rapidly applying a positive voltage.

For reactive targets, poisoning of targets must be taken into consideration. This may occur when the surface composition of the sputtering target is altered. In the case of $\mathrm{Nb}$, which oxidizes rapidly under ambient conditions, the target must be cleaned prior to use. Additionally, many oxides are dielectric materials, so as a positive charge accumulates on the target, ion bombardment will slow, and given a large enough electrostatic imbalance, a dielectric breakdown may occur. When cleaning poisoned targets or attempting to sputter dielectric materials, like $\mathrm{MgO}$ in the case of the work presented here, a pulsed power supply is advantageous.

In the present work, surface treatments consisting of homoepitaxial growth of $\mathrm{MgO}$ 
on commercial $\mathrm{MgO}$ substrates films were carried out via reactive sputtering to stabilize epitaxial $\mathrm{Nb} / \mathrm{MgO}$ thin film growth. Reactive sputtering was also used in related $\mathrm{NbN}$ work featured elsewhere $[11,36,37]$.

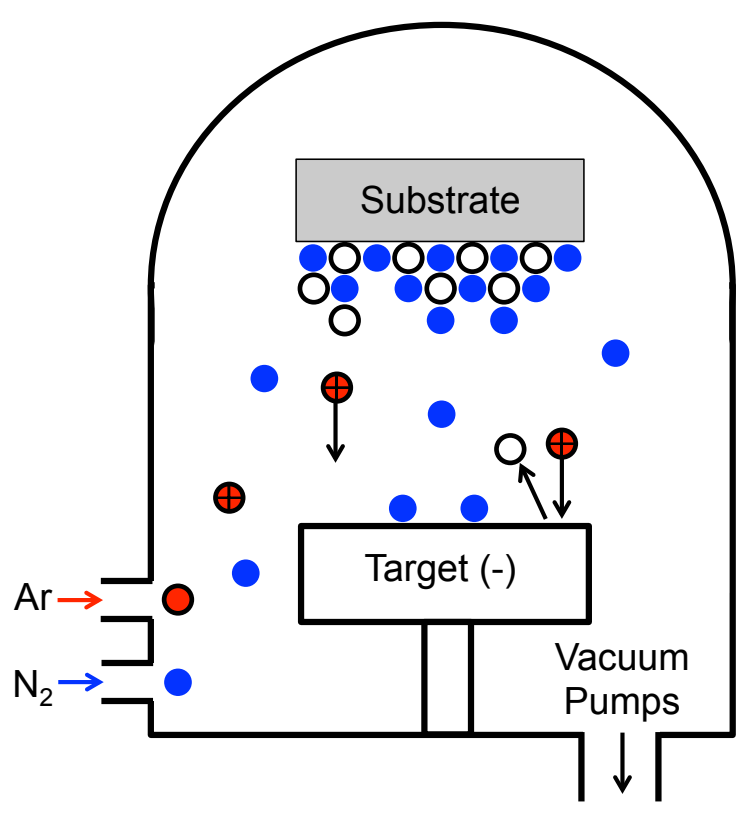

FIG. 4.2: Schematic illustrating a reactive magnetron sputtering process.

\subsubsection{Hybrid Physical-Chemical Vapor Deposition of $\mathrm{MgB}_{2}$ Thin Films}

Currently, hybrid physical-chemical vapor deposition (HPCVD) is a favored technique for the deposition of stoichiometric, high-quality $\mathrm{MgB}_{2}$ thin films. HPCVD is similar to other CVD deposition techniques with the inclusion of a means to heat a solid metallic target material, such as $\mathrm{Mg}$, in close proximity to the substrate. Thermal energy is applied to the metallic target material to achieve high vapor pressure of the target material proximal to the substrate surface, which reacts with a precursor gas (a mix of $H_{2} B_{2} H_{6}$ in this 
case), forming a condensate which collects on the substrate. Some HPCVD systems allow for independent control of substrate temperature as well as the metallic source allowing for additional thermodynamic control of phase.

The difficulty of stabilizing $\mathrm{MgB}_{2}$ thin films via other deposition techniques, such as magnetron sputtering, stems from the thermodynamic requirements of the $\mathrm{Mg}$ - $\mathrm{B}$ system. For films with stoichiometric uniformity, a high temperatures and a high saturation partial pressure of $\mathrm{Mg}$ is required to stymie the formation of $\mathrm{MgB}_{4}$ and $\mathrm{Mg}_{7}$ ( $10 \mathrm{mTorr}$ ), thus excluding deposition techniques requiring high or ultra-high vacuum conditions, such as sputtering or MBE [38].

All of the samples featured in this work were provided by our collaborators at Temple University, successful pioneers in the production high-quality, epitaxial $\mathrm{MgB}_{2}$ thin films. A thickness series of epitaxial $\mathrm{MgB}(0001)_{2} / c-\mathrm{Al}_{2} \mathrm{O}_{3}(40,60,80$, and $100 \mathrm{~nm})$ was prepared using the HPCVD method outlined above. Each film features a thin Au capping layer $(\sim 10$ $\mathrm{nm})$ to mitigate degradation of the sample surface during transport and characterization in ambient conditions.

\subsection{Thin Film Characterization}

The following sections outline the primary tools used in the characterization of thin film samples featured in this dissertation. Atomic Force Microscopy (AFM) was used to characterize surface morphology. Reflection High-Energy Electron Diffraction (RHEED) and X-ray Diffraction (XRD) were used to characterize the microstructure of the epitaxial thin films. Magnetic measurements were obtained using a Superconducting Quantum Interference Device (SQUID). 


\subsubsection{Atomic Force Microscopy}

AFM belongs to a class of scanning probe microscopy techniques akin to scanning tunneling microscopy (STM). AFM measures the "atomic" forces between a very sharp probe tip and a sample surface to detect changes of surface topography on the order of angstroms. One advantage of AFM over STM is that a conducting sample is not required since the feedback control is not reliant upon a tunneling current between the probe and the sample, hence the topography of insulating materials may also be imaged. The spatial resolution is typically lower than for STM, however in specifically-prepared systems and well-controlled environments, atomic resolution is possible. There are two common modes of operation for AFM - a contact mode (electro-repulsive forces), where the tip makes direct contact with the sample surface, and a non-contact mode where the tip and the surface are widely separated and the sample-tip system are weakly coupled by van der Waals forces. The non-contact mode was used exclusively in the work presented here, as it offers advantages of reducing damage and contamination from tip-surface contact. In non-contact mode, with relatively hard materials, such as refractory metals (such as $\mathrm{Nb}$ ), a stiff cantilever (one with a large spring constant) is usually used to prevent tip-surface contact from an attractive van der Waals force [18].

The basic AFM experiment (Figure 4.3) consists of (i) a piezoelectric scanning stage capable of 3D positioning in $x, y$ and $z$ cardinal directions with picometer precision, (ii) a force probe or cantilever whose minute deflections while in the presence of interatomic forces are observed with optical interferometry and converted into an electrical signal and (iii) an electronic control and feedback system coupling the response of the cantilever with the piezoelectric positioning.

The tip-surface interaction is monitored via an optical system consisting of a laser and photodiode detector. The probe, mounted on a reflective cantilever with a known spring 
constant, is driven near a fixed characteristic frequency (usually between 100 and 400 $\mathrm{kHz}$ ). As the tip approaches the surface of interest, changes in the cantilever vibrational amplitude, frequency, and phase relative to the driving signal are converted to voltages, recorded by software and converted into an image.

Typically in non-contact mode, the feedback controls attempt to maintain the vibrational frequency of the cantilever as the probe rasters across the surface, thereby attempting to maintain the $z$ distance between the sample and and the tip (i.e. keeping the van der Waals force constant), culminating in a false color topographical representation of the surface.

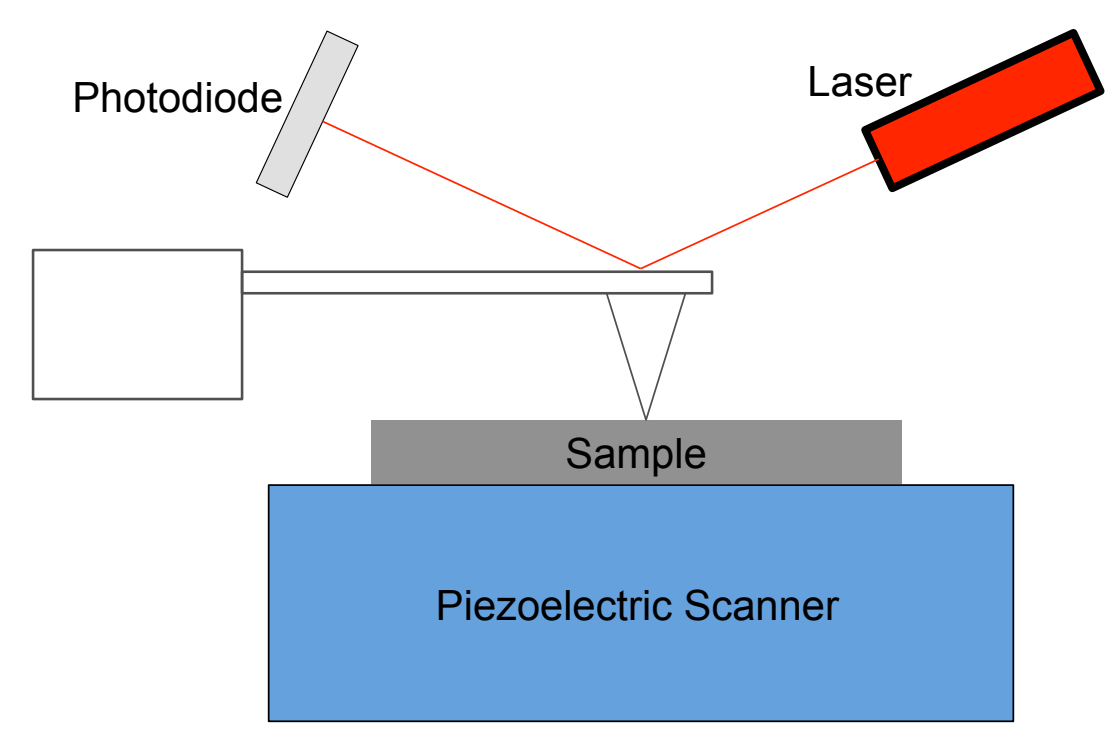

FIG. 4.3: Illustration of an AFM configuration.

Owing to the sensitive nature of the AFM experiment, it is desirable to eliminate extraneous sources of acoustic and electronic noise from the AFM system, thus vibrational isolation and electronic shielding are desirable. The Spring constant of the cantilever should be small compared to the effective spring constant between the atoms comprising the surface of interest so as not to displace surface atoms. Commercial micro-fabrication 
techniques of AFM cantilevers allow for the production of less rigid cantilevers for imaging softer materials like biological materials. Specialized magnetic probes may also be used to image the domain structure of magnetic materials in magnetic force microscopy (MFM).

\subsubsection{Diffraction Techniques}

Diffraction is an interference phenomenon which broadly arrises when waves encounter material interface boundaries. Striking diffraction effects, such as constructive and destructive interference bands, epitomized by Young's famous single and double slit experiments, occur as a result of a difference in path lengths of waves from spatially separated scattering centers. Interference patterns are most pronounced when the wavelength of the incoming wave is comparable to the periodic length scale of scattering features.

Diffraction effects may be used to probe the structural character of materials and undergirds myriad interferometry devices and techniques. The resolution limit of such techniques is largely determined by the wavelength of the probe signal. Interatomic distances in solids is on the order of a few angstroms, thus X-ray wavelengths are needed. The periodic electronic structure of crystalline solids provide the necessary boundaries such that diffraction can be used to investigate the microstructure of crystalline materials.

\section{Reflection High Energy Electron Diffraction}

Reflection high energy electron diffraction (RHEED) is a surface-sensitive diffraction technique widely used for the structural characterization of thin films. In its most primitive form, the RHEED experiment consists of an electron gun, a clean sample of interest, and a phosphorescent screen. Figure 4.4 illustrates a typical RHEED geometry. The electron beam reaches the sample at a shallow incidence angle, typically less than $5^{\circ}$, and diffracted electrons produce an interference image on a phosphor screen. At these 
glancing angles, owing to the shallow penetration depth of electrons, electrons probe only the topmost layers, thus the structural information contained within the diffraction pattern is attributable only to the microstructure of the first several atomic layers of the sample near the surface.

RHEED yields qualitative as well as quantitative information about materials, and because of its sensitivity, is particularly well-suited for surface characterization of epitaxial thin films. ${ }^{1}$ The diffracted signal is related to the in-plane periodic structure (i.e. the atomic arrangement) on the surface of the film. Owing to the vacuum requirements for operation of the electron gun, the RHEED technique is particularly compatible with MBE experiments where real-time, in situ monitoring of in-plane microstructure and film growth rates is possible.

In the case of sputtering, which requires the presence of a working gas typically in the mTorr range, RHEED may not be always be directly utilized concurrent with film growth unless differential pumping is used; however, it may be used to obtain structural information before and after growth or during thermal treatments absent sputtering. Differential pumping of the electron gun may be used to allow concurrent operation of sputtering and RHEED, provided that the mean free path of the electrons is larger than the distance between the source and the screen to minimize detrimental scattering from the ambient working gas. Because the diffraction patterns, particularly for those arising from surface reconstructing, can be quite complicated, RHEED is sometimes used in conjunction with other surface characterization techniques, such as STM, to determine microstructure.

For the purposes of this thesis, RHEED was primarily used to determine in situ the epitaxial registry between substrate and deposited thin films. For a comprehensive

\footnotetext{
${ }^{1} \mathrm{~A}$ precise understanding of system geometry is required for quantitative structural analysis with RHEED. In some cases, a relative measurement from a well-characterized surface may be enough to calibrate subsequent measurements, as in the case of determining the in-plane lattice parameter of an epitaxial film grown on a substrate.
} 


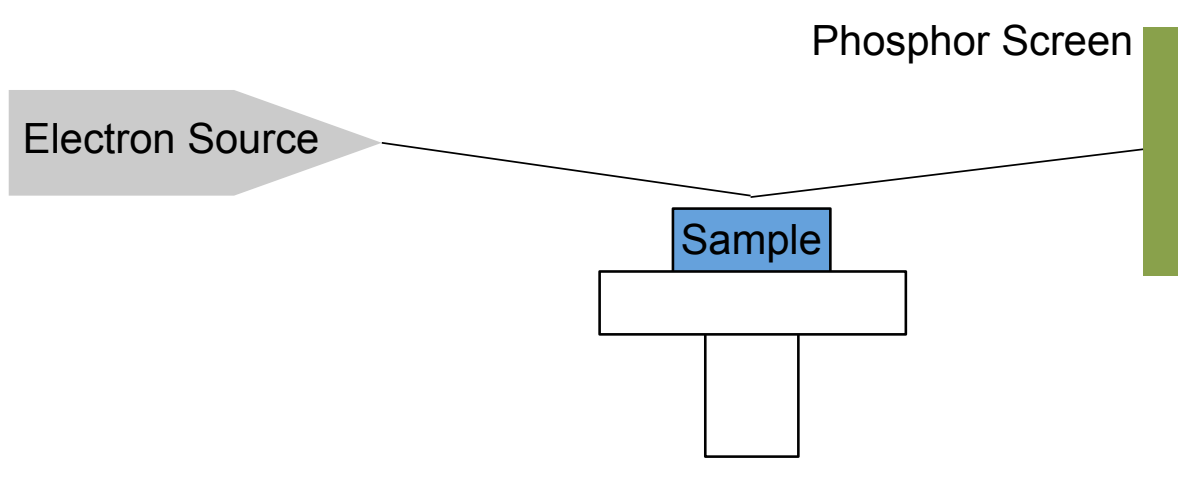

FIG. 4.4: Grazing-incidence geometry in a typical RHEED experiment.

treatment of RHEED and its applications, please refer to reference [39].

The electron gun in a RHEED system should have a small angular divergence and the beam should be focused to a small spot size on the sample. In practice, most commercial instruments utilize electrostatic and magnetic optics to collimate, focus, and steer the electron beam. A poorly collimated beam will produce an undesirable broadening in the diffracted signal. As a rule of thumb, the divergence of the electron beam should be on the order of the sample flatness (angular spread $\sim 1 \mathrm{mrad}$ ). such that the broadening of the diffracted beam is dominated by the surface disorder in the sample. In order for the diffracted signal to be sensitive enough to track subtle changes in the atomic arrangement of the sample surface, the probe's wavelength must be less than interatomic spacings. Recall that a particle's de Broglie wavelength $\lambda$ is inversely proportional to its momentum $p$

$$
\lambda=\frac{h}{p}
$$


where $h$ is Plank's constant. In relativistic terms, the relationship between a particle's momentum $p$ and energy $E$ is given by $E^{2}=(p c)^{2}+\left(m c^{2}\right)^{2}$. The total energy of an electron of mass $m_{e}$ with charge $q_{e}$ accelerated through an electric potential $V$ is $E=q_{e} V+m_{e} c^{2}$. Solving for this relativistic momentum $p$ and substituting into de Broglie's equation yields an electron wavelength

$$
\lambda=\frac{h}{\sqrt{2 m_{e} q_{e} V}} \frac{1}{\sqrt{1+\frac{q_{e} V}{2 m_{e} c^{2}}}} .
$$

The wavelength of the electron is tunable by adjusting the electric potential through which the electron is accelerated. The length scales that are of interest are on the order of interatomic spacings, thus for practical operation electron energies in the range of $10 \mathrm{keV}$ to $30 \mathrm{keV}$ are desirable. At higher electron energies, the diffracted pattern is more compressed on the phosphor screen allowing for the observation of more interference fringes. Note that our RHEED is installed in a system which also features magnetron sputtering guns, which have stray magnetic fields arising from the permanent rare-earth magnets used to confine the plasma near the target. Operating at higher electron energies mitigates the splaying of the electron beam from these stray fields. ${ }^{2}$

\section{X-Ray Diffraction}

X-ray diffraction (XRD) is a principal tool for ex situ exploring the microstructure of crystalline materials and thin films in particular. In the idealized XRD experiment, Xrays incident upon a crystalline material - i.e. where the constituent atoms are arranged in a regular, periodic manner — diffract, giving rise to observable interference patterns from which qualitative and quantitative information about structure can be obtained. The

\footnotetext{
${ }^{2}$ For these reasons, an electron energy of $29 \mathrm{keV}$, which corresponds to a wavelength $\lambda=0.07102 \stackrel{\circ}{A}$ was chosen for all RHEED experiments considered in this dissertation.
} 
diffraction conditions (Bragg conditions) are given by

$$
n \lambda=2 a \sin \theta,
$$

where $n$ is an integer, $\lambda$ is the wavelength of the x-ray, $a$ is the distance between diffraction planes and $\theta$ is the angle between the incident x-ray and the diffraction plane. Practically speaking, if the wavelength is known, by measuring the angle at which constructive interference occurs a determination of the average interatomic spacing of diffraction layers can be made. Equation 4.3 is valid when $\lambda \leq 2 d$. In order to observe interference effects on a length scale comparable to interatomic distances, the X-ray must probe length scales on the order of interatomic distances. Laboratory XRD instruments generate X-rays by accelerating electrons ejected from a hot filament towards a target material, like $\mathrm{Cu}$, with sufficient kinetic energy to knock out core shell electrons. When valence electrons move to occupy the vacancy, a characteristic X-ray is emitted ( $\mathrm{Cu} \mathrm{K}$-alpha with a wavelength $0.15418 \mathrm{~nm}$ for the purposes of the studies contained in this dissertation). Various optical components of the XRD machine are used to focus and condition the X-rays into a quasi-parallel monochromatic beam which illuminates the sample of interest.

For thin films, it is important that the XRD machine be equipped with a four circle goniometer (Figure 4.5) to allow for proper alignment and optimization of signal intensity. Often in epitaxial thin films, a slight angular misalignment can manifest between the diffraction planes of the substrate and the diffraction planes of the thin film. Hence, access to additional angles of rotation allow an operator to optimize on the thin film signal (typically much weaker than bulk crystalline substrate signatures).

Important structural information that may be accessed includes orientation of crystal planes, relative crystallographic orientation of the substrate (for understanding epitaxy), in-plane and out of plane lattice parameters, relative strain, crystallite size, mosaic struc- 


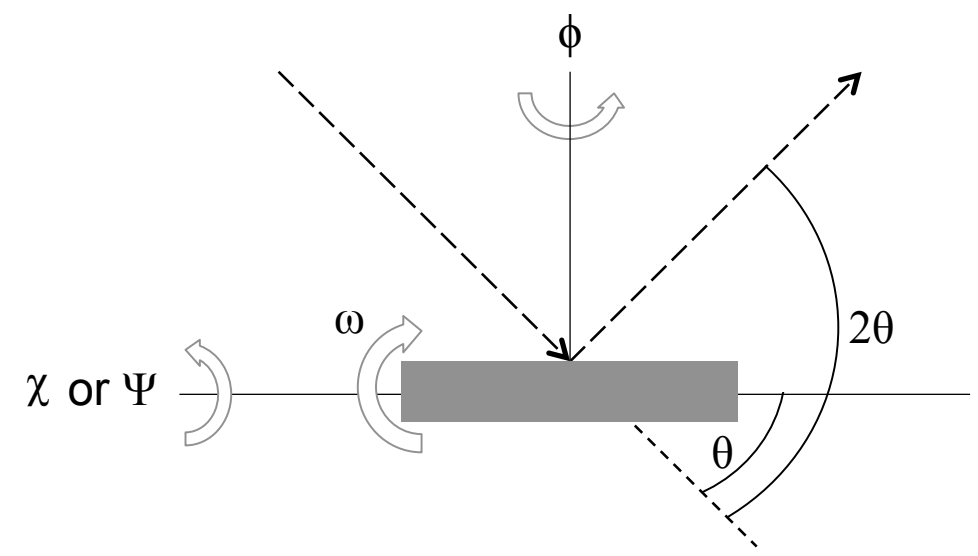

FIG. 4.5: Four circle goniometer or Eulerian Cradle. $\theta$ (X-ray source) and $2 \theta$ (detector) can be independently positioned. $\omega$ has a rotation axis normal to the $\theta-2 \theta$ plane. $\phi$ has $360^{\circ}$ of azimuthal rotation about the axis normal to the sample surface. $\chi$ (sometimes referred to as $\Psi)$ rotates about an axis normal to the axes of rotation for $\omega$ and $\phi$.

ture as well as more complex inverse maps and pole figures. In the most basic experiment, a powder-diffraction reference file is consulted for phase identification. In a typical High Angle $(\theta-2 \theta)$ scan, in-plane lattice parameters associated with the thin film may be calculated from Bragg's law and compared with standard powder-diffraction reference samples to determine strain as a percent difference.

$$
\text { Strain }=\frac{d-d_{b u l k}}{d_{b u l k}}
$$

From $\theta-2 \theta$ scans, the average crystallite or grain size $L$ may be estimated using the Scherrer equation, 


$$
L=\frac{k \lambda}{B_{r} \cos \theta}
$$

where $k$ is a geometric constant dependent on the symmetries of the crystal lattice (approximately 0.94 for cubic systems) and $B_{r}$ is the corrected Full Width Half Maximum (FWHM) of the Bragg diffraction peak. Due to imperfect optics, the FWHM of the diffracted beam is influenced by the structure of the film as well as intrinsic instrumental broadening. Instrumental broadening can be determined and corrected for with the use of a crystalline standard, typically a single crystal Si wafer.

\section{Rocking curves}

So-called single crystal thin films may not be perfectly continuous over the entire plane of the sample. Strain is relieved in the form of grain boundaries between individual crystallites. Rocking curves are used to determine the degree to which these crystallites are ordered, thus rocking curves give an idea of mosaic structure, or long-range order of the crystalline thin film. In practice, this is accomplished by fixing the angle of the source and detector and rocking the sample in the $\omega$ direction. Crystalline films exhibiting a high degree of long-range order will have narrow FWHM about this $\omega$ scan.

\section{XRR for film thickness}

$\mathrm{X}$-ray reflectometry is a grazing incidence application of XRD which is primarily used to determine interfacial thickness, interfacial roughness, and density in thin film samples. It should be noted that this technique may be applied to amorphous films as crystalline onesthe interference patterns arise from interfacial distances rather than interatomic distances. 
An XRR plot looks at the intensity of the reflected beam as a function of $\theta$ over a shallow range ( $\sim 0-5$ degrees). The signature is then fit using algorithms that take into account interfacial models based upon variations from bulk values for thickness, interface roughness and electron density.

\subsection{Magnetic Characterization via SQUID}

SQUIDs (Superconducting Quantum Interference Device) serve as very sensitive magnetometers allowing for precise control of temperature and applied magnetic fields. These devices have found common use in a range of applications to characterize the magnetization and susceptibility of materials over a range of temperatures. Many hallmarks of superconducting compounds are observed as magnetic phenomena, thus SQUIDs are well suited for the study of superconducting materials. A Quantum Design MPMS XL system was used for this research. An optimally calibrated system can produce applied fields from -7 to $7 \mathrm{~T}$ and may resolve magnetic moments on the order of $10^{-15} \mathrm{~T}$ with well-controlled sample temperatures from 2 - $400 \mathrm{~K}$ (temperatures lower than $4.2 \mathrm{~K}$, the boiling temp of liquid helium, supplied by pumping mechanisms).

The broad features of the SQUID magnetometer include a temperature control system, a superconducting electromagnet control system, a SQUID, and a sample manipulation system. $\mathrm{L}_{\mathrm{He}}$ serves as the "fuel" for these devices, providing the means for temperature control in the sample space as well as serving as the cryogen necessary for superconducting components central to the device.

A SQUID is a superconducting loop with either one (AC SQUID) or two (DC SQUID) Josephson Junctions placed along the path of a superconducting loop. Here we outline the basics of DC SQUID and AC SQUID. Josephson junctions are two superconductors weakly coupled with a thin - as compared to the coherence length of Cooper pairs - 
insulating layer which serves as a tunneling barrier. There is a critical current $I_{C}$ above which a voltage appears across the junction.

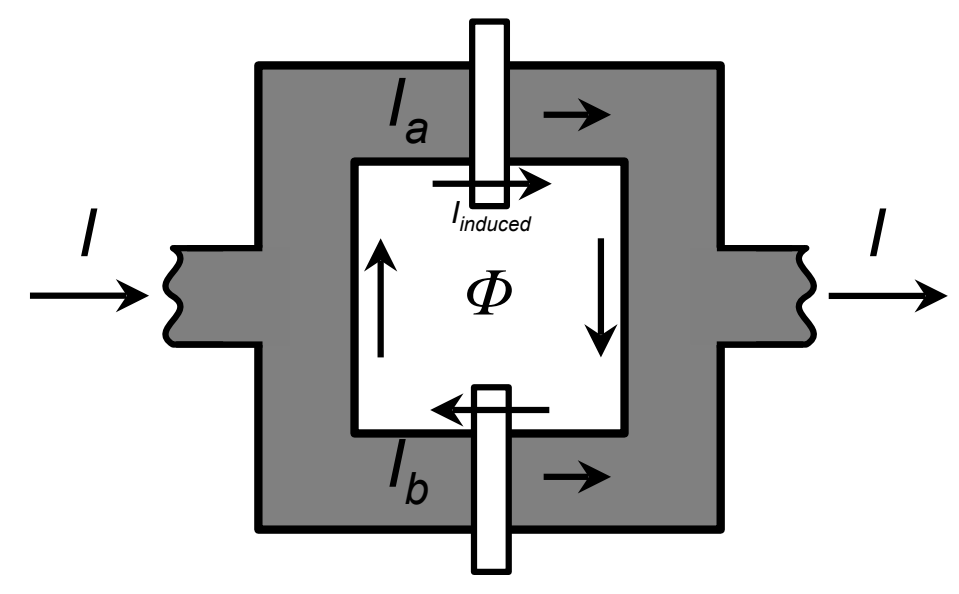

FIG. 4.6: DC SQUID device.

The "quantum" aspect of the SQUID comes from the recognition that magnetic flux is quantized and that the flux through the SQUID must be a integer number of the flux quanta $\Phi_{0}$. In the absence of an applied field, an applied subcritical biasing supercurrent is divided evenly between the two Josephson junctions. With a small amount of flux increase (less than $\Phi_{0} / 2$ ), the SQUID establishes a screening current in the superconducting loop to oppose and cancel the applied flux, reducing the current in one branch while increasing the current across the other above $I_{c}$, which is observed as a change in voltage $V$ across the Josephson Junction. As the flux increases above $\Phi_{0} / 2, \Phi_{0}$ is now more energetically favorable, thus the current (no longer screening) reverses direction to ensure that the flux passing through the SQUID is now $\Phi_{0}$, which is now observed as $-V$ across the junction. Thus, the SQUID converts changes in magnetic flux to voltage oscillations. When the flux in the loop is increased, the voltage oscillates with a period of $\Phi_{0}$, thus a total change in 
flux can be determined by counting voltage oscillations.

In operation, a magnetic sample is translated through a series of superconducting pickup coils in discrete steps. As the sample is passed through the pickup coils, an induced current is established in the pickup coils which is then converted via the SQUID to voltages, which is interpreted by standard fitting algorithms (approximating the sample as a point dipole source) to determine the magnetic moment of the sample.

The cylindrical geometry of the MPMS limits the dimensions of the samples to approximately $5 \mathrm{~mm}$ diameter cylinders. Typical thin film samples examined in our studies had dimensions around $5 \mathrm{~mm} \times 5 \mathrm{~mm} \times 0.5 \mathrm{~mm}$. We note that thin film samples can be oriented either perpendicular or parallel with respect to the applied field $H$ within the sample space. Because of the geometry of the accelerating modes in SRF cavities, for the purposes of this study we are primarily interested in orientations where the applied field is parallel to the surface of the film.

AC SQUID differs from DC operation in that an alternating applied field is superimposed upon a DC field, providing additional phase information. The frequency-dependent complex susceptibility can be expressed as $\chi(\omega)=\chi^{\prime}(\omega)+i \chi^{\prime \prime}(\omega)$, where the real part $\chi^{\prime}(\omega)$ describes the behavior of the system in phase with the incoming $\mathrm{AC}$ field and the imaginary part $\chi^{\prime \prime}(\omega)$, which accounts for the energy losses within the sample. The MPMS allows AC frequency tuning in ranges from 0.00035 to $1512.1 \mathrm{~Hz}$ (far lower than the $\mathrm{GHz}$ frequencies commonplace in SRF resonant cavities.)

\subsubsection{Determining $T_{C}$ and $H_{C 1}$ of Superconducting Thin Films}

In the case of superconducting materials, the MPMS can be used to determine properties such as $T_{C}$ by exploiting the Meissner effect. To measure $T_{C}$, the sample first undergoes a zero field cool (ZFC) where, absent an applied field, the sample is cooled well 
below an expected transition temperature (a minimum of $2 \mathrm{~K}$ for the MPMS). Recall that superconductors in the presence of a weak applied field (below $H_{C 1}$ ) exhibit a diamagnetic response, thus by applying a weak field (on the order of 10 Oe) we can observe the diamagnetic response as a function of sample temperature. The temperature of the sample is gradually increased until a temperature $T_{C}$, where the magnetic moment approaches zero and the sample is returned to a normal conducting state.

$H_{C 1}$ measurements are considerably more involved and must be carefully considered. Superconductors in the Meissner state behave as perfect diamagnets, so the magnetic response is proportional to the applied field. $H_{C 1}$ is often defined as the field at which the superconducting sample magnetic response deviates from the characteristic diamagnetic "Meissner Slope," the reversible portion of the $H$ curve absent flux pinning. A sample first undergoes a ZFC to a temperature below $T_{C}$, a field is applied and measurement of the magnetic response recorded. The response of the sample is collected over a range of field values and $H_{C 1}$ is taken to be the lowest field value where the response deviates from perfect diamagnetic behavior.

The above procedure suffers from over estimates of $H_{C 1}$ in cases where flux pinning is observed. An alternate method for determining $H_{C 1}$ is finding the lowest field value where magnetic flux is trapped in the superconducting sample. The procedure, outlined by Bohmer et al. may be used to this end. (1) First, the sample undergoes a ZFC into the superconducting state and a measurement at zero field is taken, serving as a background measurement. (2) A measurement is taken with an applied field and the sample response is measured again - essentially replicating $\mathrm{M}(\mathrm{H})$ approach outlined above. (3) Finally, the applied field is removed and the magnetic response is taken again and then sample temperature is returned to a normal state to clear any trapped fields for subsequent measurements. Subtracting the measurement (3) from measurement (1), a non-zero result suggests trapped fields and hence a departure from Meissner state into the 
irreversible mixed phase. Measurement (2) serves as a sanity check, essentially reproducing the traditional $\mathrm{M}(\mathrm{H})$ profile to check against the trapped flux method.

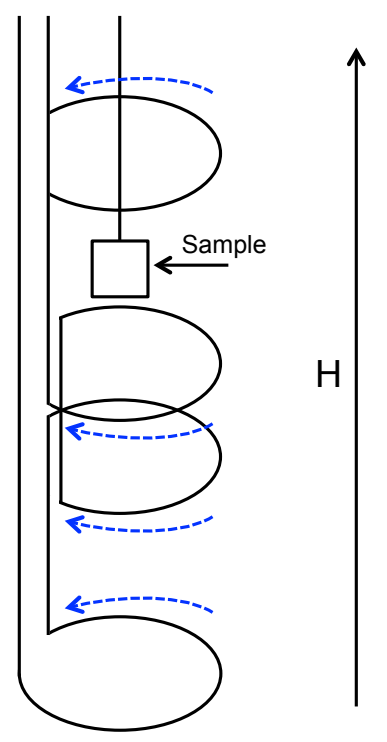

FIG. 4.7: Geometry of MPMS SQUID experiment. The sample is passed through a series of superconducting pickup coils whose induced, quantized current is registered as a voltage change in the SQUID. 


\section{CHAPTER 5}

\section{Structure and Property Correlations}

\section{in Superconducting Thin Films}

This chapter gives results obtained in our correlated studies on epitaxial $\mathrm{Nb}$ and $\mathrm{MgB}_{2}$

thin films. The first section deals with the evolution of surface morphology for eptiaxial $\mathrm{Nb} / \mathrm{MgO}$. The following section details work with epitaxial $\mathrm{Nb} / \mathrm{Al}_{2} \mathrm{O}_{3}$. The final section looks at $\mathrm{MgB}_{2} / \mathrm{Al}_{2} \mathrm{O}_{3}$ and correlates film thickness and film morphology with observed enhancements in $H_{C 1}$.

\subsection{Epitaxial Nb Grown on $\mathrm{MgO}(001)$ Surfaces}

It has been observed that epitaxial Nb thin films deposited onto crystalline insulating surfaces can evolve in very different fashions depending on specific deposition conditions, thereby affecting microstructure, surface morphology and superconducting properties. In this section we examine and contrast the microstructure and ensuing surface morphology from two distinct $\mathrm{Nb} / \mathrm{MgO}$ growth series each with its own epitaxial registry - namely 
$\mathrm{Nb}(001) / \mathrm{MgO}(001)$ and $\mathrm{Nb}(110) / \mathrm{MgO}(001)$ — leading to distinct surface anisotropy, and we closely examine the dynamical scaling of the surface features during growth. The findings are compared with other metal/MgO epitaxial systems and a general scaling formalism is applied to analyze anisotropic surfaces exhibiting biaxial symmetry.

This formalism, described in Chapter 3, is reliant upon the addition of an independent scaling parameter in Fourier space, acquired from a Power Spectral Density (PSD) plot, which is applied to the specific problem of thin film growth and surface evolution, to describe the scaling universality class under a General Dynamic Scaling formalism. Owing to the distinct surface evolution of $\mathrm{Nb} / \mathrm{MgO}$ stemming from slight changes in growth parameters, this analysis aims to qualify a set of deposition conditions leading to smoother surfaces in $\mathrm{Nb} / \mathrm{MgO}$ systems. Overall, the surface morphology and microstructure of the various Nb films correlate well with superconducting and normal state figures of merit such as $H_{C 1}$ and RRR.

The following sections describe the growth and evolution of the microstructure and surface morphology of $\mathrm{Nb}$ films grown on $\mathrm{MgO}(001)$ substrates using DC magnetron sputtering and the application of models for roughness evolution to investigate the $\mathrm{Nb} / \mathrm{MgO}$ system in question. That resultant surface morphology is, in fact, a consequence of the particular microstructure of the $\mathrm{Nb}$ film and of the epitaxial relationships between the $\mathrm{Nb}$ film and $\mathrm{MgO}$ substrate, supported by a strong structure-morphology correlation. Note that the general scaling formalism described in Chapter 3 is quite general and therefore applicable to films obtained using other promising energetic-condensation thin-film deposition techniques, such as ECR, and offers a suitable comparison platform for disparate deposition approaches.

The task of understanding and quantifying the dynamic evolution of growth fronts is translated into questions regarding the relative spatial and temporal scaling relationships between similar, persistent, characteristic features, and their associated length scales 
throughout the development of the surface - specifically, the correlation between certain characteristic lengths both parallel and perpendicular to the direction of the evolving growth front. Presented here are studies on thin film growth and dynamic evolution of the $\mathrm{Nb}$ surface under specific deposition conditions onto an insulating $\mathrm{MgO}(001)$ surface. We examine the dynamic scaling behavior of the surface roughness in two $\mathrm{Nb} / \mathrm{MgO}$ series with differing epitaxial registries, $\mathrm{Nb}(001) / \mathrm{MgO}(001)$ and $\mathrm{Nb}(110) / \mathrm{MgO}(001)$. Utilizing Power Spectral Density (PSD) analysis, a General Dynamic Scaling model is applied to extract additional scaling parameters to determine a universality classes for epitaxial $\mathrm{Nb} / \mathrm{MgO}(001)$ thin films [40]. Furthermore, we correlate our findings with relevant transport and superconducting properties such as residual resistance ratio (RRR) and $H_{C 1}$. The work contained within this section has been published previous in References [7, 9].

\subsubsection{Experimental Details}

Epitaxial $\mathrm{Nb}$ films were grown on $\mathrm{MgO}(001)$ substrates in an UHV deposition system with a base pressure around $9.0 \times 10^{10}$ Torr. The deposition system used for this study is a modified Perkin-Elmer MBE system with 5 source ports outfitted with sputtering guns and additional cryopumping to enable adequate pumping speed for sputtering process in the low to mid mTorr range. Films were prepared via DC magnetron sputtering of a 2-inch diameter $\mathrm{Nb}$ target of $99.95 \%$ purity. Prior to deposition, each polished $\mathrm{MgO}$ substrate was ultrasonically cleaned in subsequent 10 minute baths of acetone and methanol. A pre-deposition in situ annealing treatment for 1 hour at $600^{\circ} \mathrm{C}$ was carried out in order to degas and recrystallize the surface layers of each substrate, and the subsequent growth of each film was carried out at a temperature of $600{ }^{\circ} \mathrm{C}$.

Two separate Nb film thickness series, with film thicknesses ranging from 10 to 1000 nm, were prepared under similar deposition conditions. Series 1 films (designated as those 
demonstrating a bi-crystalline structure) were deposited using DC magnetron sputtering with an Ar working gas pressure of 1.0 mTorr. Series 2 films (demonstrating a single crystal structure) were sputtered at 5.0 mTorr. The DC power delivered to the Nb target was $60 \mathrm{~W}$. Growth rates for the thin films, as determined by XRR, were $0.32 \AA / s$ for Series 1 and $0.48 \AA / s$ for Series 2 .

Since the initial substrate surface is an important parameter affecting the subsequent film growth, in the case of the $\mathrm{Nb}(100) / \mathrm{MgO}(001)$, it was found necessary to deposit a thin and fresh seed layer of $2 \mathrm{~nm}$ of $\mathrm{MgO}$, via reactive sputtering, onto the substrate using reactive sputtering in order to augment the initial growth surface and minimize surface issues that could hinder the desired epitaxy. Recall that substrate temperature and deposition rates are dominant factors in determining the rate and distribution of nucleation events in thin films. Note also that higher working gas pressure and increased sputtering power - tunable parameters that also influence the growth of the single crystal films - both lead to a corresponding increase in the growth rate (Series 2), and serve as the primary distinction between Series 1 and Series 2 films.

RHEED was employed mainly to obtain in situ information regarding the epitaxial registry of the $\mathrm{Nb}$ films on $\mathrm{MgO}$, as well as the quantify the strain evolution and verify the presence of surface faceting on $\mathrm{Nb}$ films deposited on sapphire as well as $\mathrm{MgO}$ substrates. Characteristic RHEED patterns were obtained for each film along two distinct crystallographic directions corresponding to the $\mathrm{MgO}[100]$ and $\mathrm{MgO}[110]$ azimuths. In order to establish an understanding of mosaic structure of the thin films, additional structural characterization was obtained via XRD rocking curves as described in Chapter 4.

Ex situ AFM was used for the characterization of the surface morphology for roughness evolution studies. AFM images were collected with a Nanotec Cervantes AFM. The AFM was operated in non-contact/tapping mode with an AppNano ACTA Si-N AFM tip that has a nominal tip diameter of $10 \mathrm{~nm}$. The free software WSxM from Nanotec was used 
for the analysis of the AFM images [41]. Line scans, self-correlation functions, Fourier transforms, root mean square (RMS) roughness, and PSD functions were used in the processing and analysis of the AFM images. Line scans in conjunction with $2 \mathrm{D}$ selfcorrelation were utilized to extract global and local anisotropic lateral correlation lengths (i.e., average island size and separation) as well as RMS information over short length scales, RMS roughness data, representing the surface interface width, were obtained from the AFM topography images. The WSxM software features a PSD package which used to determine the average spectral features of the thin films.

\subsubsection{Characterization of Structure and Morphology}

Figure 5.1 (a) illustrates the observed epitaxial registry for $\mathrm{Nb} / \mathrm{MgO}$. RHEED images (Figures 5.1(d) and 5.1(e)) from Series 1 taken along two distinct azimuthal directions in the $\mathrm{Nb} / \mathrm{MgO}$ system indicate an epitaxial, mixed $\mathrm{Nb}$ structure, where $\mathrm{Nb}(110) / \mathrm{MgO}(001)$ is present with two possible in-plane azimuthal crystalline orientations, $\mathrm{Nb}[110] \| \mathrm{MgO}[100]$ and $\mathrm{Nb}[110] \| \mathrm{MgO}[010]$. Each of the films throughout this particular thickness series shares these structural characteristics.

In contrast, the RHEED images (Figures 5.1(b) and 5.1(c)) from Series 2 show a single crystal $\mathrm{Nb}$ structure with $\mathrm{Nb}(100) / \mathrm{MgO}(001)$ where $\mathrm{Nb}[100] \| \mathrm{MgO}[100]$. The observation that $\mathrm{Nb}$ achieves these particular registries with the underlying $\mathrm{MgO}(001)$ substrate is consistent with previous reports [42]. The determination of which particular epitaxial $\mathrm{Nb}$ registry is achieved between Series 1 and Series 2 films appears to be highly sensitive to the initial substrate conditions (i.e., miscut, domain distribution, and roughness) as well as the growth rate and sputtering pressure. To explain the observed correlation between growth conditions and the texture of the films, we note that similar results were reported for Pt films grown on $\mathrm{MgO}$ [43]. 

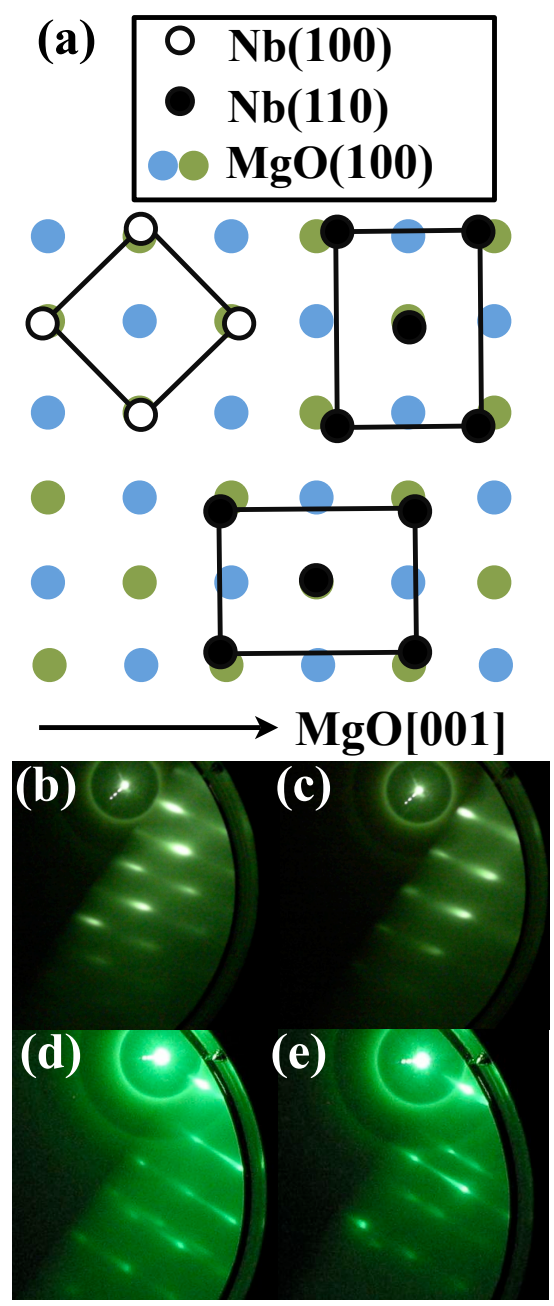

FIG. 5.1: $\mathrm{Nb} / \mathrm{MgO}$ epitaxial relationships as verified by RHEED. (a) Atomic representation of epitaxial registry of $\mathrm{Nb}$ films with respect to the $\mathrm{MgO}(001)$ substrate. (b) RHEED image of $100 \mathrm{~nm}$ single crystal $\mathrm{Nb}(001) / \mathrm{MgO}(001)$ taken along the $\mathrm{MgO}[001]$ azimuth and (c) along the

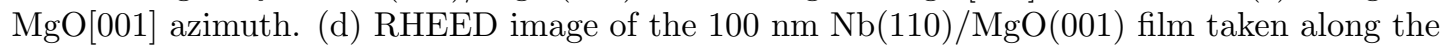
$\mathrm{MgO}[011]$ azimuth and (e) along the $\mathrm{MgO}[001]$ azimuth. 


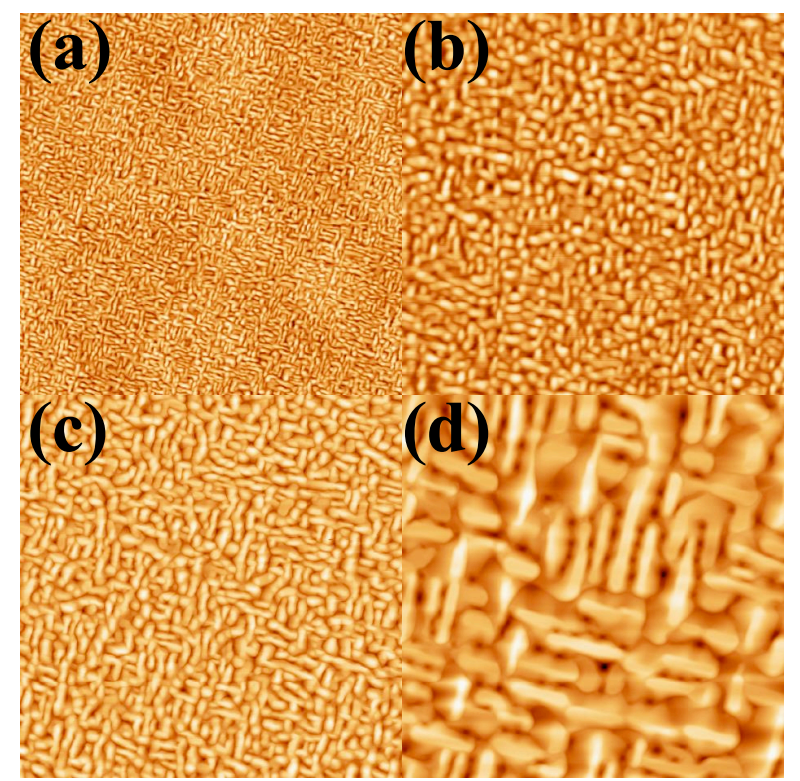

FIG. 5.2: Representative $2 \times 2 \mu \mathrm{m}$ AFM scans of Nb thin films from Series 1 for thicknesses of (a) 30 (b) 50 (c) 100, and (d) $600 \mathrm{~nm}$.

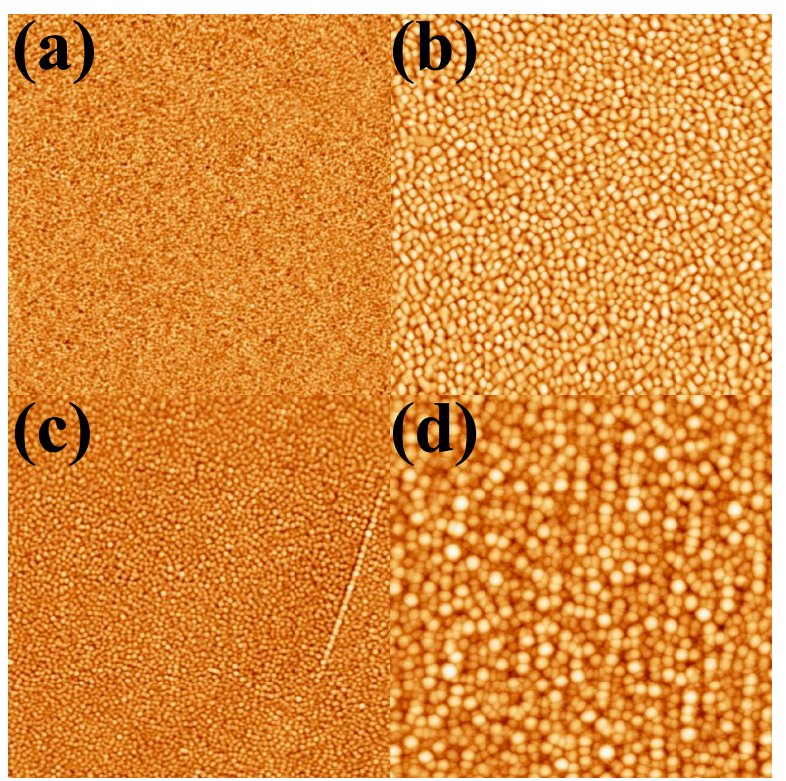

FIG. 5.3: Representative $2 \times 2 \mu \mathrm{m}$ AFM scans of Nb thin films from Series 2 for thicknesses of (a) 30 (b) 50 (c) 100, and (d) $600 \mathrm{~nm}$. 
Due to the large lattice mismatch between $\mathrm{Nb}$ and $\mathrm{MgO}$, the specific epitaxial relationships observed for $\mathrm{Nb}$ films on $\mathrm{MgO}$ substrates can be interpreted in the frame of the domain matching epitaxy or an extended atomic distance mismatch approach [44]. The idea here is that $m$ integer lattice units of the film match with $p$ integer lattice units of the substrate, i.e., $m d_{f} \approx p d_{s}$ or $\left(d_{f} / d_{s}\right) \approx(p / m)$, with $d_{f}$ and $d_{s}$ being the respective interatomic spacing in the film and substrate along parallel directions. Assuming bulk values of $d_{f}$ and $d_{s}$ for $\mathrm{Nb}$ and $\mathrm{MgO}$, it results that for $\mathrm{Nb}(001) / \mathrm{MgO}(001), m=11$ and $p=10$ with a lattice mismatch around $0.7 \%$. Moreover, the minimum in-plane size of the $\mathrm{Nb}$ domains on $\mathrm{MgO}$ is $3.3 \mathrm{~nm}$.

In the case of $\mathrm{Nb}(110) / \mathrm{MgO}(001)$, i.e., the bi-crystalline structure, two in-plane relationships exist with two lattice mismatchs $(2 \%$ and $0.7 \%$ ), and the minimum in-plane size of the $\mathrm{Nb}$ domain on $\mathrm{MgO}$ is about $4.6 \mathrm{~nm}$. From such values, it is clear that the $\mathrm{Nb}$ domains in the bi-crystalline structure $(4.6 \mathrm{~nm})$ require that the $\mathrm{Nb}$ species have enough time to diffuse in the direction of the major morphological anisotropy axis, adequate time diffusion times being obtained with a relatively low deposition rate.

The $\mathrm{Nb}$ isotropic domain in the $\mathrm{Nb}(001) / \mathrm{MgO}(001)$ structure $(3.3 \mathrm{~nm})$ needs a lower in-plane diffusion of the species, and thus a higher deposition rate is required to suppress diffusion rates and to stabilize this particular epitaxy. Note that this corresponds well with observations on the relationship between the empirically determined deposition rates and resulting film epitaxy.

The ensuing surface morphology of the films is a correlated directionally with the observed epitaxy and was investigated using AFM. The fast scanning direction in each AFM scan was chosen parallel to the $\mathrm{MgO}[001]$ direction. In Series 1, a clear biaxial morphological anisotropy is manifest and persistent throughout the thickness series (Figure 5.2). Suggestively, the direction and relative orientation of the morphological anisotropic features are consistent with structural anisotropies observed in the corresponding RHEED 
patterns. In stark comparison, the AFM scans from Series 2 (Figure 5.3) show a more isotropic surface morphology devoid of the interwoven, anisotropic island features observed in Series 1 films.

To further correlate the surface morphology with the microstructure of the films, XRD was employed to determine the characteristic mosaic structure for each film thickness series. Rocking curves about the $\mathrm{Nb}(011)$ reflection in Series 1 films revealed multiple mosaic domains, whereas the rocking curves about the $\mathrm{Nb}(002)$ reflection in Series 2 films were characterized by a single mosaic domain. These observations are consistent with the morphological and structural domains obtained with AFM and RHEED. As determined from the FWHM of rocking curves, films from thickness Series 2 films were generally possessed of comparatively broad mosaic dispersion $\left(0.1^{\circ}-1.0^{\circ}\right)$ than Series 1 counterparts $\left(0.1^{\circ}-0.5^{\circ}\right)$. It should also be noted that the deviant $50 \mathrm{~nm}$ film from Series 2, which does not follow the prevailing RMS roughness vs. thickness trend, had a comparatively large mosaicity $\left(\sim 1^{\circ}\right)$.

\subsubsection{Determination of Scaling Exponents}

A comparison of the RMS surface roughness for the thickest film $-6.7 \mathrm{~nm}$ for the 600 $\mathrm{nm}$ film from Series 1 and $5.9 \mathrm{~nm}$ for the 1,000 $\mathrm{nm}$ film from Series 2 - in addition to the striking differences in the observed morphological anisotropy provides an initial indication that dynamic scaling parameters are not necessarily identical between the two thickness series.

In order to investigate the roughness scaling features, the global scaling parameters were extracted from the AFM scans from each thickness series. The 2D auto-correlation function in WSxM was applied to the AFM images and provided a means for identifying the anisotropic average island size by fitting the average features to Gaussians and calculating 
their FWHM value [45]. For Series 1, the nature of the observed anisotropy is biaxial; hence, the autocorrelation function, which was taken along the fast scan direction, is taken to be a convolution of the average island size along the major and minor anisotropy axes. As such, it is necessary in this case to define two separate dynamic scaling parameters. Fitting a convolution of Gaussians allows for the determination of global, anisotropic values for $\beta / \alpha$. The values $(\beta / \alpha)_{\text {width }}=0.40$ and length $(\beta / \alpha)_{\text {length }}=0.55$ as well as $\beta=0.60$ were found from linear fits from the relevant log-log plots (Figures 5.4(a) and 5.4(b)). Anisotropic values for global $\alpha$ were calculated to be $\alpha_{\text {length }}=1.1$ and $\alpha_{\text {width }}=1.5$ with an average $\alpha_{\text {avg }}=1.3$, which was derived from a radial average of the auto-correlation function.

Local scaling parameters (Figures 5.4(c) and 5.4(d)) for Series 1 were obtained via real space methods by analyzing short line scans constrained along the tops of the morphological islands. The local values $\alpha_{l o c}=0.56$ and $\beta_{l o c}=0.34$ were obtained (see Figure 5.5). Here, we see clear deviation between the local and calculated global scaling parameters adhering to the anomalous scaling universality class as indicated by Equation 3.5.

Scaling parameters for Series 2 films were similarly extracted from the corresponding AFM images. Due to the isotropic nature of the surface features, only average features were considered for the determination of scaling parameters. A global coarsening parameter $\alpha=4.3$ was calculated from the measured scaling parameters of $\beta / \alpha=0.31$ and $\beta=1.3$ (Figure 5.6).

To correlate our assessment based on microstructure and surface roughness analysis with physical properties relevant to the proposed application in SRF cavities, we have also measured relevant superconducting properties, since it is expected that the crystalline structure, in particular the defects (atomic defects, extended defects, and grain boundaries), play a major role in the superconducting properties. Thus, we have determined the RRR, which is the ratio of resistance at $300 \mathrm{~K}$ (room temperature) to the resistance 
(a)

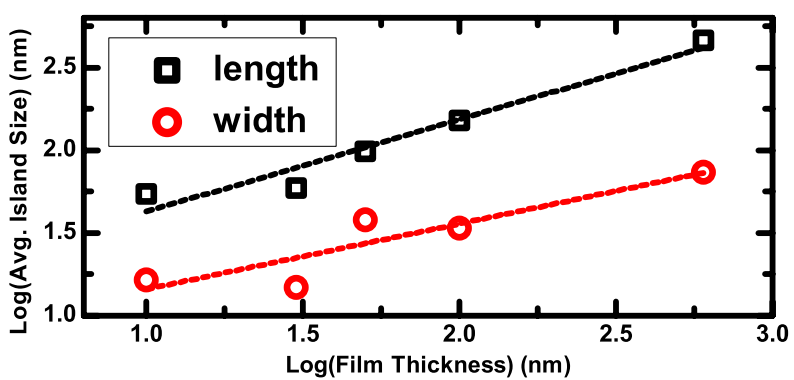

(b)

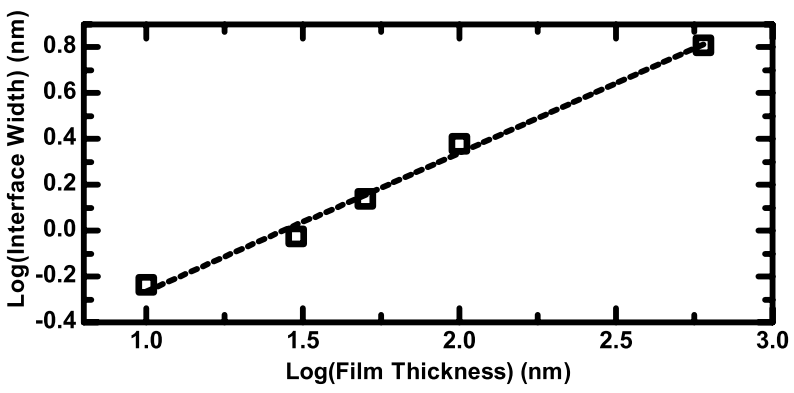

(c)

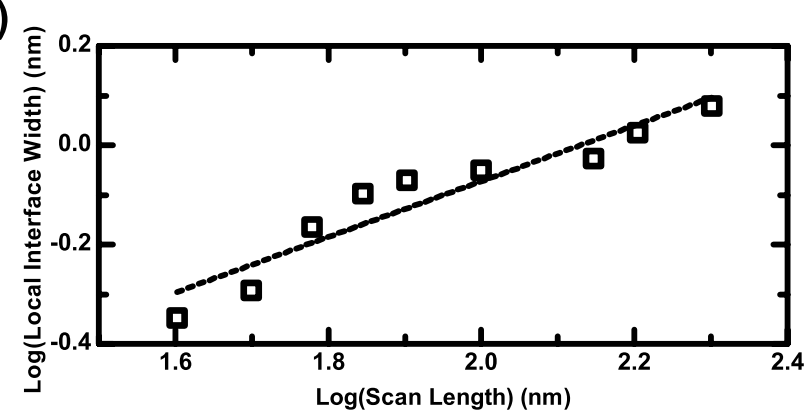

(d)

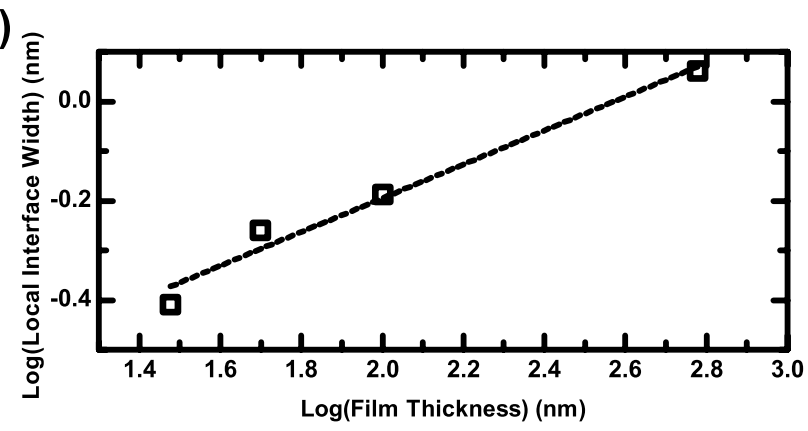

FIG. 5.4: Logarithmic scaling plots from Series 1 films of (a) island size (anisotropic) vs. film thickness (b) global interface width vs. film thickness (c) local interface width vs. scan size, and (d) local interface width vs. film thickness. 


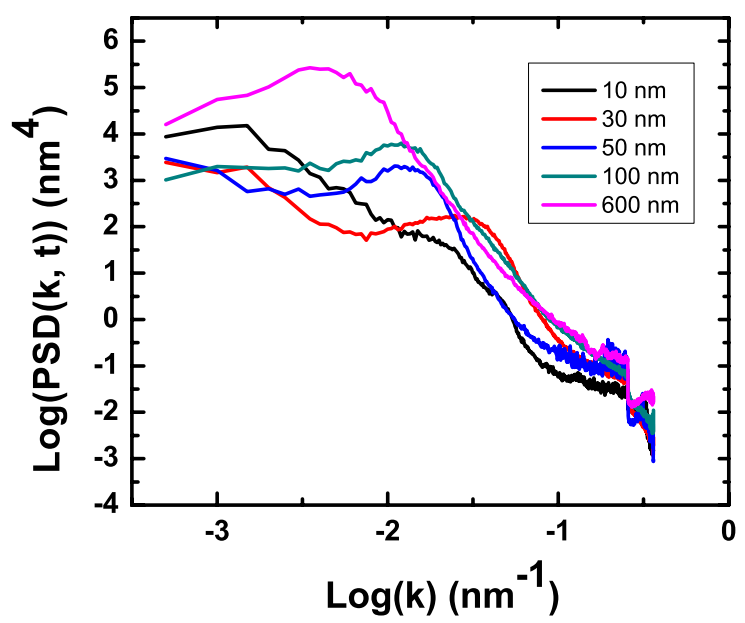

FIG. 5.5: Power Spectral Density curves for Series $1 \mathrm{Nb}$ films.

at $10 \mathrm{~K}$ (slightly above $T_{C}$ ). Lower values of RRR indicate a greater concentration of imperfections, and for that reason the value of RRR is in general used to qualify the low-temperature thermal conductivity of $\mathrm{Nb}$ films for SRF applications. Here, these measurements were carried out using a 4-point probe setup in the 6 - $300 \mathrm{~K}$ temperature range for representative films with similar thickness $(600 \mathrm{~nm})$ grown with the characteristic morphological and structural characteristics from each series. Figure 5.7 shows the results for one such typical RRR measurement for a $600 \mathrm{~nm}$ film from Series 2. The $T_{C}$ for the niobium films was found to be $\sim 9.2 \mathrm{~K}$. We found that RRR data are consistent with our surface assessment, since lower RRR values were obtained for rougher and non-crystalline films; thus the RRR values were 46.5 for the Series 1 film and 165.5 for Series 2 films. We note that the high $\mathrm{RRR}$ values reported for $\mathrm{Nb}$ films also correspond to mono-crystalline epitaxial $\mathrm{Nb}$ films deposited onto $\mathrm{MgO}(001)$ [46]. Epitaxial films from Series 2 tend towards smoother surfaces and higher RRR compared to the epitaxial thin films in Series 1. 
(a)

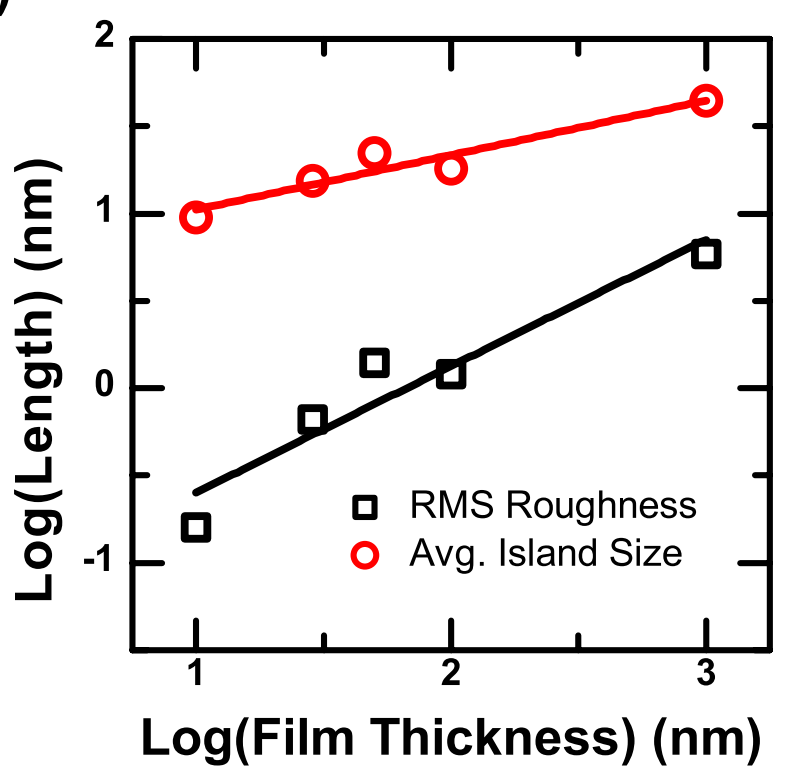

(b)

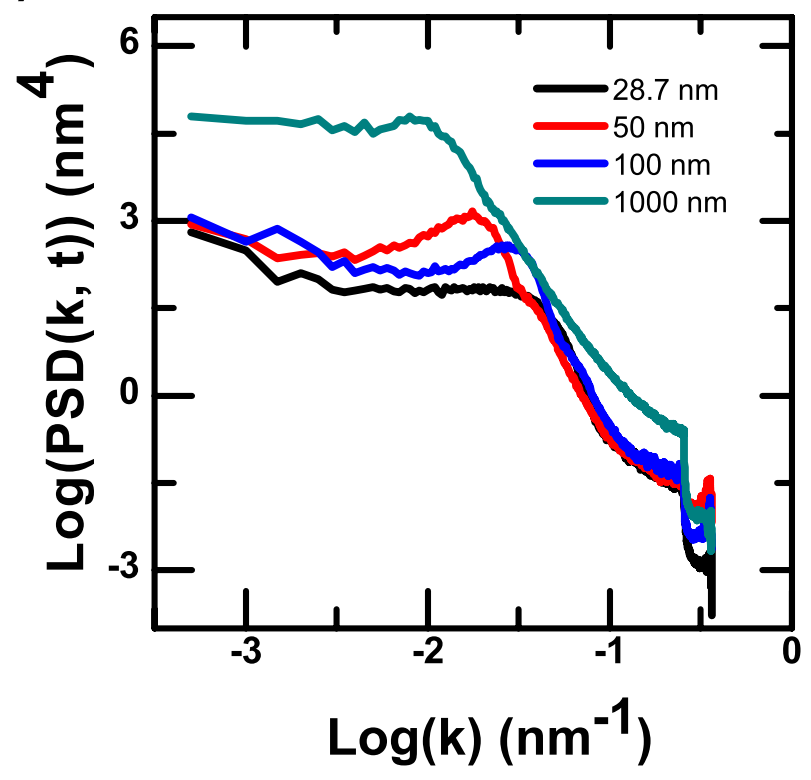

FIG. 5.6: Logarithmic plots for Nb thickness Series 2 of (a) RMS Roughness and Average Island Size vs. Film Thickness and (b) Power Spectral Density as a function of spatial frequency k. 


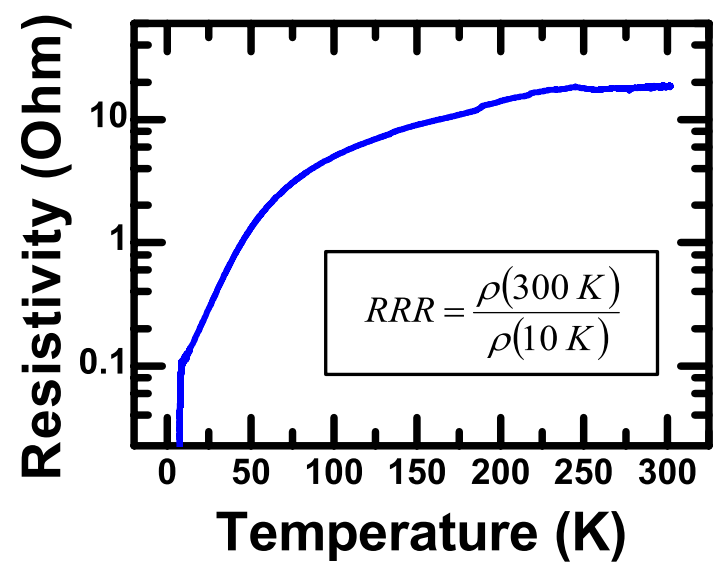

FIG. 5.7: Resistivity as a function of temperature measured for a $600 \mathrm{~nm}$, single crystal $\mathrm{Nb}(001) / \mathrm{MgO}(001)$ film.

Values for the lower critical field $H_{C 1}$ were probed using DC SQUID magnetometry for a few representative samples sharing the structural and morphological traits characteristic of Series 1 and Series 2 films. Detailed results correlating the superconducting properties of $\mathrm{Nb}$ thin films epitaxially grown on a variety of substrates are fully presented in Ref [36]. Table 5.1 shows the results of these $H_{C 1}$ measurements for $\mathrm{Nb}$ films with a range of thicknesses for both $\mathrm{Nb}(001) / \mathrm{MgO}(001)$ and $\mathrm{Nb}(110) / \mathrm{MgO}(001)$ films. The precise method implemented to determine accurate $H_{C 1}$ values for these films is presented in Ref [47]. For the present study, it is worth noting that films from Series 2 are characterized by larger $H_{C 1}$ values when compared to than those from Series 1 of comparable thicknesses. Thin films typically have a higher density of defects than bulk materials that can inhibit superconducting properties [48]. Hence, an increase in defect density may inhibit entry into the superconducting phase, effectively lowering critical fields in films with comparable thickness. Thus, the correlation of microstructure and surface roughness with $H_{C 1}$, as well as RRR, indicates that epitaxial mono-crystalline $\mathrm{Nb}$ films exhibit comparatively better performance than epitaxial bi-crystalline $\mathrm{Nb}$ films. 
TABLE 5.1: Summary of superconducting properties of Nb thin films. $T_{C}$ values were measured with an applied field of 50 Oe. $H_{C 1}$ values were measured at $4 \mathrm{~K}$.

\begin{tabular}{ccccc}
\hline \hline & $\mathrm{Nb}(100)$ & $\mathrm{Nb}(100)$ & $\mathrm{Nb}(110)$ & $\mathrm{Nb}(110)$ \\
Thickness & $T_{C}(\mathrm{~K})$ & $H_{C 1}(\mathrm{Oe})$ & $T_{C}(\mathrm{~K})$ & $H_{C 1}(\mathrm{Oe})$ \\
\hline 100 & 9.2 & 1300 & 9.2 & 600 \\
$500-600$ & 9.2 & 1600 & 9.3 & 1200 \\
1000 & 9.3 & 1800 & 9.2 & 1700 \\
\hline \hline
\end{tabular}

$\mathrm{Nb}$ films, with differing epitaxial registries with respect to the underlying substrate, exhibit similar $T_{C}$ performance, comparable to that of bulk Nb; however, present results also indicate that epitaxial mono-crystalline $\mathrm{Nb}$ films exhibit comparatively better performance with respect to $H_{C 1}$ and RRR than epitaxial bi-crystalline Nb films. Similar studies on of high $T_{C}$ superconducting films indicate that high quality thin films have a high degree of orientation, a low mosaicity, an absence of secondary phases and growth directions, and a low defect content. Thus, these results are consistent with the observation that microstructure and ensuing surface properties, i.e. surface roughness, substantially impact the superconducting quality of the films.

In summary, it has been shown that the epitaxial growth of $\mathrm{Nb}$ films onto $\mathrm{MgO}(001)$ substrates can exhibit one of two distinct crystallographic registries - $\mathrm{Nb}(110) / \mathrm{MgO}(001)$ with two possible azimuthal crystalline domains corresponding to $\mathrm{Nb}[110] \| \mathrm{MgO}[100]$ and $\mathrm{Nb}[110]|| \mathrm{MgO}[010]$ as well as $\mathrm{Nb}(001) / \mathrm{MgO}(001)$ where $\mathrm{Nb}[100] \| \mathrm{MgO}[100]$ - depending on early nucleation and growth conditions. The degree of crystalline microstructure of the films can be explained in the frame of the domain matching epitaxy, while the ensuing correlated morphology anisotropies and dynamical evolution of the surfaces can be analyzed within the context of General Dynamical Scaling. For the given growth parameters, the $\mathrm{Nb}(001) / \mathrm{MgO}(001)$ surface scales as a "super-rough" universality class and the $\mathrm{Nb}(110) / \mathrm{MgO}(001)$ surface scales as a "new-class" universality class. Pursuant to practi- 
cal considerations for the development of multilayers, the relatively low RMS roughness of the $\mathrm{Nb}$ surface for thick films of $\mathrm{Nb}(001) / \mathrm{MgO}(001)$, as compared with the thick films of $\mathrm{Nb}(110) / \mathrm{MgO}(001)$, suggests it may be a more desirable candidate in terms of minimizing surface roughness. Furthermore, the correlation between superconducting properties, microstructure, and surface morphology of these films also point to improved superconducting properties in the case of single domain $\mathrm{Nb}$ films.

\subsection{Strain Effects on the Crystal Growth and Super- conducting Properties of Epitaxial Nb Ultrathin}

\section{Films}

Superconducting ultrathin films (films with thicknesses of only a few monolayers) grown epitaxially onto crystalline substrates exhibit strained epitaxial growth due to lattice mismatch. This mismatch can have a significant effect on superconducting properties. This chapter presents a correlation of the surface morphology, crystal growth, strain, microstructure, and superconducting properties in single-crystal $\mathrm{Nb}(110)$ thin films sputter deposited on $a$-plane sapphire substrates. Notably, the lattice mismatch between $\mathrm{Nb}$ and sapphire induces the formation of a hexagonal surface structure during the first three atomic layers. Subsequent growth proceeds, giving a strained $b c c \mathrm{Nb}(110)$ phase whose in-plane lattice parameter relaxes to bulk-like values. Similar lattice relaxation was also observed in the direction perpendicular to the interface using XRD and confirming with transmission electron microscopy (TEM). Significant perpendicular strain in films up to $30 \mathrm{~nm}$ thick was found to ultimately affect the superconducting properties of the $\mathrm{Nb}$ thin films as illustrated with $\mathrm{AC}$ susceptibility measurements, where dissipative effects in the lattice - associated with the presence of strain and attendant defects - were identified. 
The work outlined in this section was initially published in Reference [8].

\subsubsection{Epitaxial Nb Thin films on $a$-plane $\mathrm{Al}_{2} \mathrm{O}_{3}$}

Special attention needs to be devoted to the microstructure of superconducting thin films to achieve the desired properties for SRF applications. Specifically, multiple aspects such as crystalline quality, film strain, grain size, film thickness or the presence of defects need to be considered, many of which are predominantly determined at the nucleation stage of film growth. All of these factors can drastically influence the superconducting properties of the films such as transition temperature, critical field, and AC susceptibility.

For the case of $\mathrm{Nb}$, epitaxial thin films can be achieved on a broad range of ceramic as well as metallic materials such as $\mathrm{MgO}$, GaAs, InAs, Ta, Mo, Co, and $\mathrm{Cu}$ [49]. A match between the lattices of $\mathrm{Nb}$ and sapphire has been established for a number of different substrate orientations, with a unique three-dimensional relationship between the microstructure of the substrate and the thin film. Thus, Nb has the same orientation relative to the sapphire substrate for all of the known deposition planes, a property that was described as three-dimensional registry [50].

Sapphire serves as a prototypical system for metal on ceramic nucleation studies and produces epitaxial $\mathrm{Nb}$ films of high crystalline quality. Indeed, high quality films have been obtained on $a$-plane sapphire, where $\mathrm{Nb}(110)$ grows epitaxially with $\mathrm{Nb}[\overline{1} 00] \| \mathrm{Al2O} 3[0001]$ leading to a lattice mismatch of $10.7 \%$ along the $\mathrm{Nb}[001]$ direction and of $8.3 \%$ along the perpendicular $\mathrm{Nb}[110]$ direction. As mentioned previously, such a mismatch can be accommodated by strain in the epitaxial layer, resulting in a coherent interface, or by the formation of misfit dislocations leading to semicoherent interfaces where coherent regions are separated by misfit dislocations [51].

Following the Matthews and Blakeslee formalism for misfit dislocations, Grier et al. 
predicted a critical thickness of $7.2 \mathrm{~nm}$ for $\mathrm{Nb}$, above which misfit dislocations are favorable [52]. They reported the presence of misfit dislocations in $\mathrm{Nb}(110) /$ a-sapphire films for thicknesses larger than $8 \mathrm{~nm}$ using TEM. Nevertheless, the strain evolution during the early stages of growth, where coherent interface strain relaxation is expected, remains largely unexplored. Oderno et al. showed a transition from an initial hexagonal surface structure for thicknesses below $1.5 \mathrm{~nm}$ to bcc $\mathrm{Nb}(110)$ for thicker films; although, they did not report whether or not there is further evolution of the lattice parameters with increasing thickness corresponding to coherent strain relaxation, which is addressed in the present study [53].

The study of $\mathrm{Nb}$ thin films nucleation and growth is of paramount importance as the presence of additional phases, coherent relaxation of the strain, misfit dislocations, or other epitaxial growth defects can strongly affect superconducting properties. Presented here is a study relating superconducting properties of $\mathrm{Nb}$ thin films epitaxially deposited on a-plane sapphire with correlations of morphology and microstructure. The surface morphology was investigated using AFM, while the structure of the films was investigated using in situ RHEED and ex situ XRD and TEM. Finally, the superconducting properties of the films were investigated using DC and AC susceptibility measurements.

\subsubsection{Experimental details}

$\mathrm{Nb}$ thin films were prepared by DC magnetron sputtering deposition in the modified Perkin-Elmer ultrahigh vacuum (UHV) system, as described in Chapter 4, with a base pressure in the low $10^{-10}$ Torr range. $a$-plane sapphire substrates with miscut angles lower than $0.05^{\circ}$ were chosen to minimize the substrate morphological effects during growth The substrates were ultrasonically cleaned and subsequently annealed at $600{ }^{\circ} \mathrm{C}$ for 1 hour in UHV conditions prior growth in order to degas and improve the crystallinity of 
the substrate surface. Sputtering deposition was carried out from a high-purity (99.95\%) $\mathrm{Nb}$ target at $5 \times 10^{-3}$ Torr of Ar pressure, resulting in a growth rate of $0.35 \AA / \mathrm{s}$. To improve the deposited film homogeneity, the substrates were azimuthally rotated around the normal axis at a constant speed of $12 \mathrm{rpm}$ during growth. Nb films of thicknesses up to $600 \mathrm{~nm}$ were deposited at a substrate temperature of $600{ }^{\circ} \mathrm{C}$, which was determined to favor crystalline ordering.

RHEED analysis was performed to verify and monitor the crystalline structure of substrate and films during growth. XRD studies were performed ex situ using a standard four-circle diffractometer with $\mathrm{Cu} \mathrm{K} \alpha$ radiation to further investigate the crystallinity of the films and to identify the different phases present. High-resolution TEM data were collected with a CCD camera using a cold field emission gun TEM operated at $200 \mathrm{kV}$. The surface morphology of the samples was characterized ex situ using AFM in non-contact mode using Si-N type cantilevers with a resonance frequency of $322.9 \mathrm{kHz}$ and a nominal tip radius around $10 \mathrm{~nm}$. DC and $\mathrm{AC}$ susceptibility measurements were carried out with an MPMS SQUID magnetometer.

\subsubsection{Surface Morphology of $\mathrm{Nb} / \mathrm{Al}_{2} \mathrm{O}_{3}$ Thin Films}

The surface morphology of superconducting thin films is of particular interest in SRF applications since it can impact surface impedance and therefore SRF performance. Again, smooth films (those devoid of sharp morphological features) are desired to minimize possible field-emitting surface structures. The surface morphology can also offer further clues suggestive of possible multiple in-plane lattice orientations in heteroepitaxial growth.

AFM topography images (Figure 5.8) show the evolution of the morphology of $\mathrm{Nb}$ films deposited on a-plane sapphire with thicknesses ranging from 30 to $600 \mathrm{~nm}$. Elongated surface features along the two principal directions of the $\mathrm{Nb}(110)$ surface, $\mathrm{Nb}[001]$ and 
$\mathrm{Nb}[110]$, are observed for thinner films (Figure 5.8 (a), (b)). Two-dimensional (2D) fast Fourier transform (FFT) maps, embedded in the upper right corner of the images, further illustrate the characteristic 2-fold anisotropy. For thicker films, one anisotropy direction ultimately prevails, and trenches along the major anisotropy axis are observed (Figure 5.8 c). In addition to the observed morphological anisotropy, the roughness throughout the Nb thickness series increases monotonically with Nb (Figure $5.8(\mathrm{~d})$ ). The line profiles along random directions of the samples evolve from $0.5 \mathrm{~nm}$ rms roughness for a $30 \mathrm{~nm}$ thick film to $4.5 \mathrm{~nm}$ rms roughness for a $600 \mathrm{~nm}$ thick film, showing that no apparent saturation of the surface roughness occurs over the range of film thicknesses investigated.

Similar morphology has been observed for similarly MBE-grown $\mathrm{Nb}(110)$ films on $a$-sapphire. In this case, fingered structures were found along the $\mathrm{Nb}[110]$ direction, attributing the observed surface structure to an energy balance where substrate miscut, deposition temperature, faceting, pinholes, and lattice mismatch play [54]. This indicates that different intrinsic growth factors can affect the microstructure and the surface morphology of the films; thus sensitivity to initial substrate and deposition conditions may strongly dictate evolution of the surface morphology.

\subsubsection{Structural Characterization of Nb/Al2O3 Thin Films}

The microstructure of the Nb films was investigated via RHEED (at various stages during growth) and with XRD and TEM after growth. As noted earlier, RHEED is especially sensitive to only the topmost atomic lattice, due to the limited penetration length of electrons in metallic films at glancing angles. Thus, it is suitable for probing in-plane interatomic spacing of the surface along different azimuthal directions; therefore, it is quite sensitive to the presence of in-plane strain. RHEED patterns were taken for $\mathrm{Nb}$ films grown on $a$-sapphire at $600{ }^{\circ} \mathrm{C}$ for thicknesses ranging from 1 to 63 atomic layers 

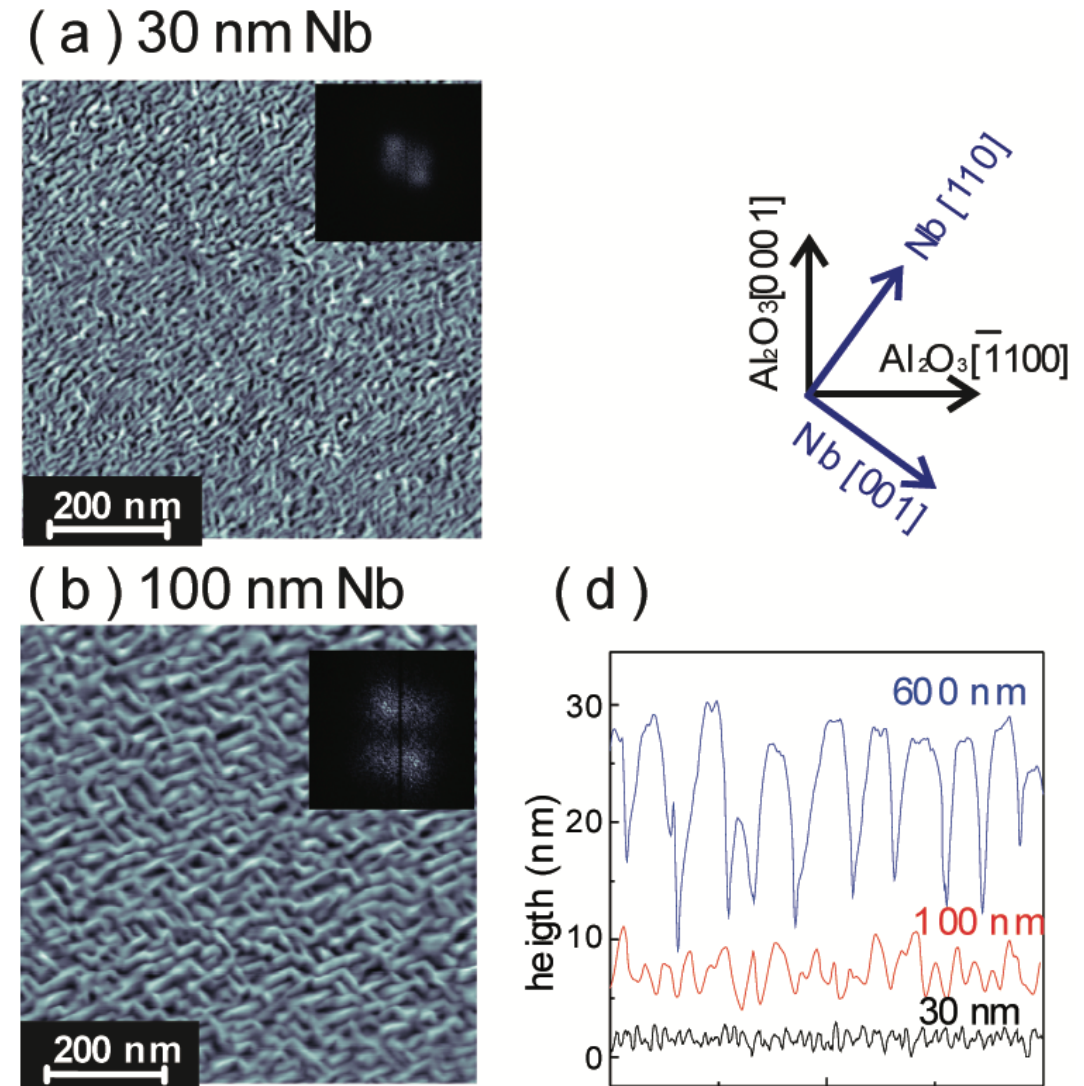

(c) $600 \mathrm{~nm} \mathrm{Nb}$
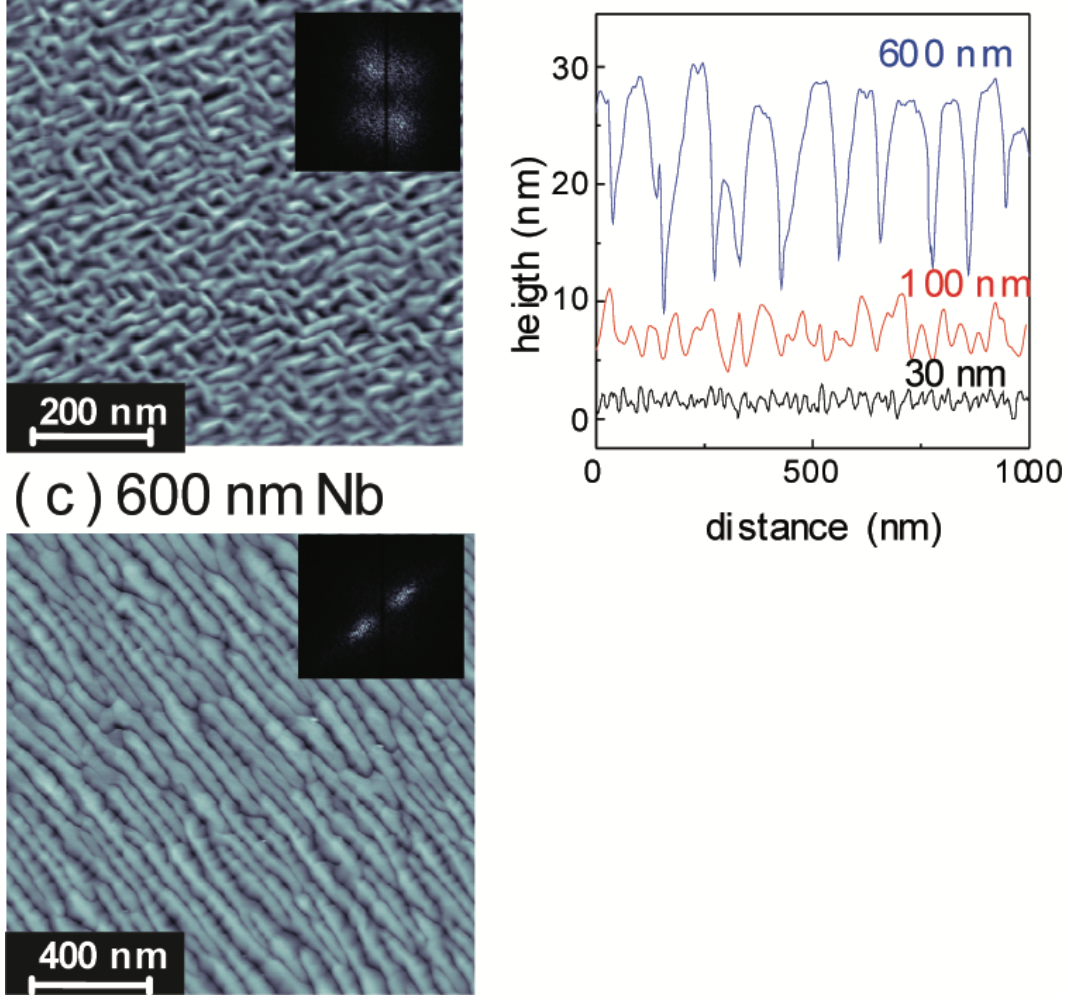

FIG. 5.8: AFM topography images for Nb films grown on $a$-plane sapphire (a) 30, (b) 100 and (c) $600 \mathrm{~nm}$. The insets on the upper right corner show 2D FFT maps showing the evolution of the morphological anisotropy. (d) Representative line scan profiles for each film demonstrate the roughening of the surface as film thickness increases. 
(0.23 - $14.5 \mathrm{~nm})$. Absent differential pumping within the deposition system, the RHEED measurements were obtained by interrupting the growth and then pumping out the Ar sputtering gas between subsequent growths.

\section{RHEED Characterization}

In the earliest stages of growth, a superposition of two distinct streak patterns that repeat with azimuthal rotation every $60^{\circ}$ are observed when the electron beam is directed along the in-plane [0001] and [1100] sapphire directions. In addition, the interline distances along those two directions have a $\sqrt{3}$ ratio, indicative of a hexagonal surface structure consistent with those previously reported [53]. Using the sapphire substrate as a crystalline reference sample, the lattice parameter for the strained hexagonal structures was extracted from the pattern found along the $\mathrm{Nb}[11 \overline{2} 0]$ direction parallel to the $\mathrm{Al}_{2} \mathrm{O}_{3}[0001]$ direction (Figure 5.9).

The initial value of $0.294 \mathrm{~nm}$ for 1 atomic layer of $\mathrm{Nb}$ decreases and stabilizes at 0.289 nm for 2 and $3 \mathrm{AL}$. With increasing thickness, the hexagonal diffraction patterns progress towards a new set of streaks corresponding to bcc $\mathrm{Nb}(001)$ orientated $\mathrm{Nb}[\overline{1} 11] \| \mathrm{Al} 2 \mathrm{O} 3[0001]$, similar in diffraction spacing but now exhibiting a rotational symmetry associated with tetragonal structure.

The coexistence of both hexagonal and tetragonal phases is found for $\mathrm{Nb}$ thicknesses ranging from 3 to $5 \mathrm{AL}(0.69-1.15 \mathrm{~nm})$. The corresponding lattice parameter for the new $b c c$ structure was measured using the pattern found along the $\mathrm{Nb}[\overline{1} 11]$ directional (parallel to the Al2O3[0001] direction in this epitaxial registry), as shown in Figure 5.9. Because of

its $b c c$ structure, the atomic plane spacing obtained from RHEED was multiplied by $2 / \sqrt{3}$ to obtain the tetragonal lattice parameter "a". A smooth evolution of the streak spacing is observed during the transition region, corresponding to gradual relaxation of atomic spacing. This precludes the onset of a strong concentration of defects in the interface between the two phases. Nevertheless, the change in structure from hexagonal to $b c c$ 


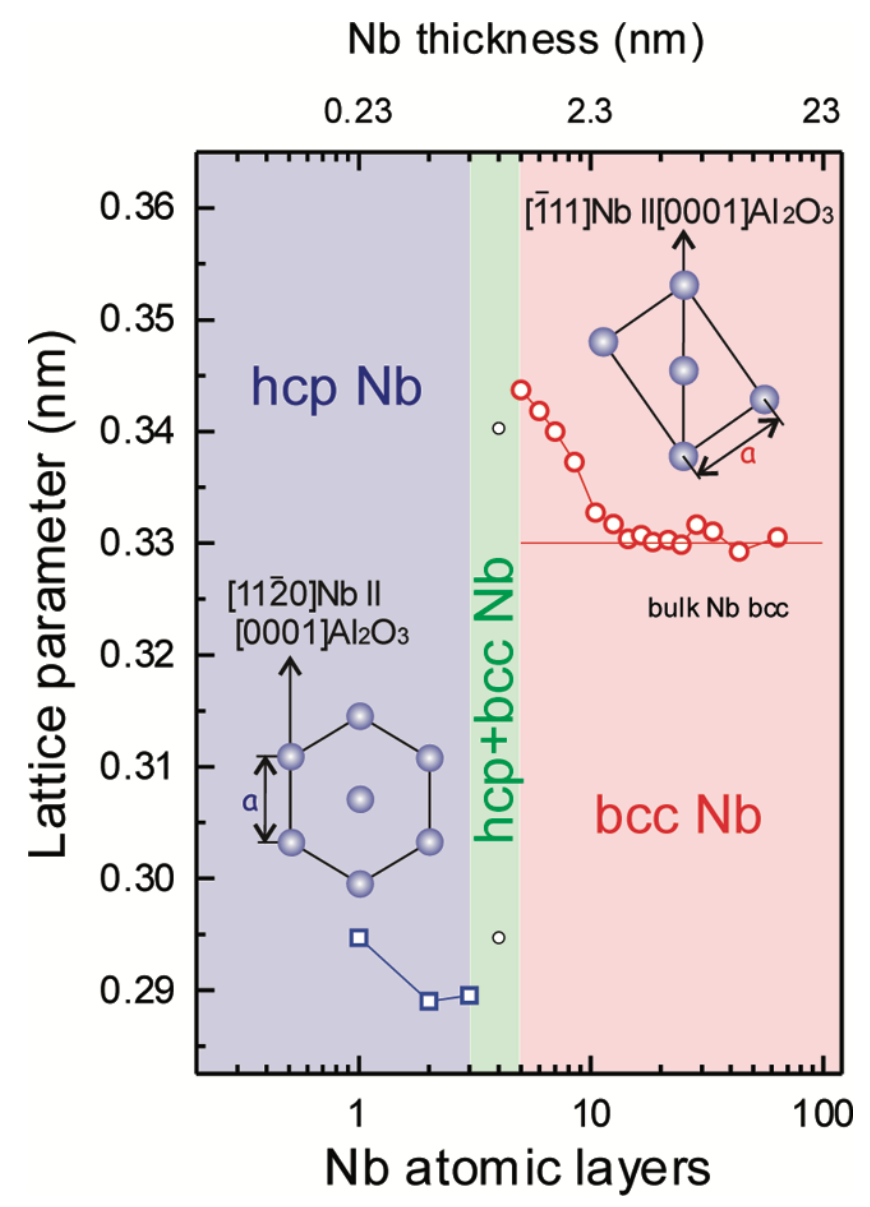

FIG. 5.9: Evolution of the $\mathrm{Nb}$ structure and lattice parameter for $\mathrm{Nb}$ films with thicknesses ranging from 1 to 63 atomic layers $(0.23 \mathrm{~nm} / \mathrm{AL})$. An initial hexagonal structure is observed for the first three atomic layers followed by a strained $b c c$ phase, whose lattice parameter relaxes after 14 atomic layers $(3.22 \mathrm{~nm})$. 
and thus the change of the observed crystallographic direction give rise to an abrupt, measurable change in lattice parameter. After the transition, an initial 3.93\% expansion of the lattice parameter as compared to bulk is observed for films 5 AL thick due to strain. This strain relaxes towards the $\mathrm{Nb}$ bulk lattice parameter $(0.33 \mathrm{~nm})$ after approximately 14 atomic layers $(3.22 \mathrm{~nm})$. RHEED analysis reveals that the $\mathrm{Nb}$ films grown on $a$ sapphire evolve via two main mechanisms to overcome the initial lattice mismatch: an initial hexagonal phase, energetically more favorable for thicknesses up to $3 \mathrm{AL}$, followed by a strained bcc $\mathrm{Nb}(110)$ phase, which progressively relaxes reaching values close to equilibrium after $14 \mathrm{Nb}$ atomic layers.

\section{XRD Characterization}

The structure and strain of the $\mathrm{Nb}$ films were also investigated ex situ via XRD. Unlike RHEED, which probes only the first few monolayers of the film, X-rays penetrate and probe the entire $\mathrm{Nb}$ film thickness (not to mention the substrate), and so the observed reflections are a convolution of the contributions arising from all of the atomic layers comprising the sample. Figure 5.10 shows symmetric XRD scans for 30, 100, and $600 \mathrm{~nm}$ thick Nb films. All of the films exhibited a single (110) phase confirming the structure observed with RHEED and their crystalline nature. Nevertheless, the position of the $\mathrm{Nb}(110)$ reflection for the $30 \mathrm{~nm}$ thick film deviates from the expected bulk position due to the lattice mismatch present between substrate and thin film, and thus, the position of such $\mathrm{Nb}(110)$ reflection is closer to the $\mathrm{Al}_{2} \mathrm{O}_{3}(11 \overline{2} 0)$ peak rather than to the corresponding bulk $\mathrm{Nb}(110)$ reflection, revealing an average "a" lattice parameter $1.25 \%$ larger than bulk $(0.33 \mathrm{~nm})$. However, the thicker films show a relaxed lattice parameter near to bulk values as can be observed for the 100 and $600 \mathrm{~nm}$ thick films, with only a $0.36 \%$ and $0.2 \%$ larger lattice parameter than bulk, respectively.

For thicker films, the relative contribution arising from the strained interface is less significant as compared to the subsequently relaxed layers, giving an average lattice pa- 


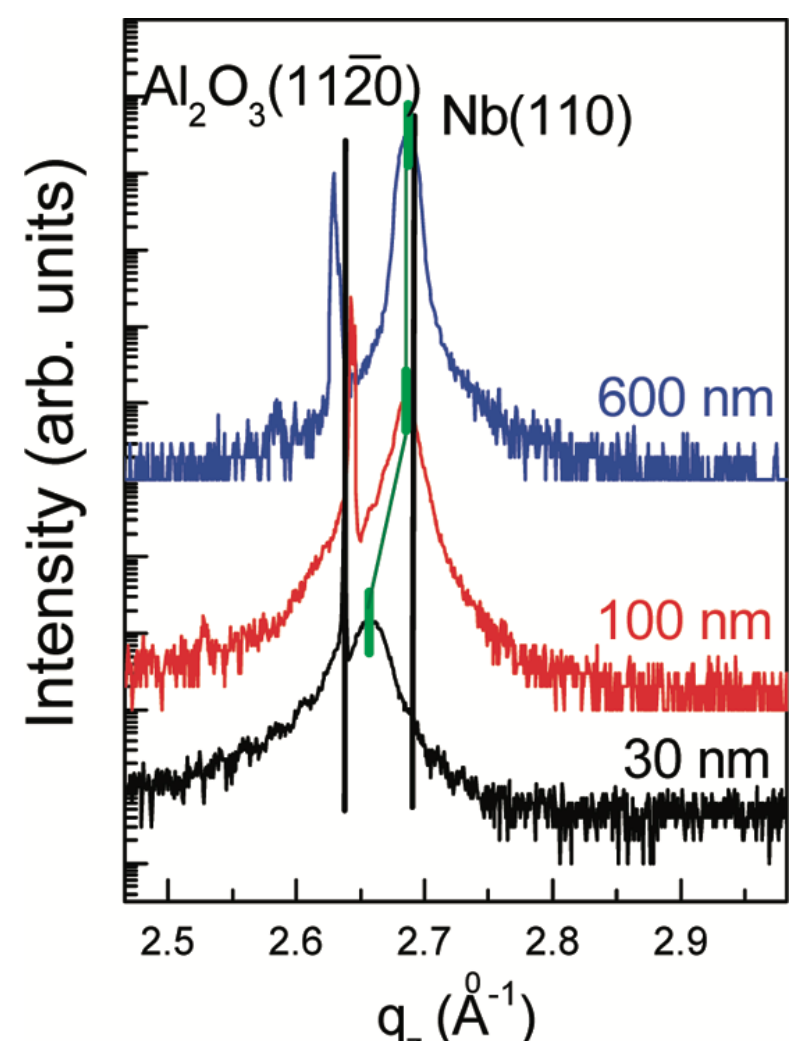

FIG. 5.10: Symmetric XRD scans for Nb films grown on a-plane sapphire 30, 100 and 600 $\mathrm{nm}$ thick. The $\mathrm{Nb}(110)$ phase is present throughout the series with an evolution of the lattice parameter toward bulk with film thickness. 
rameter very close to bulk $\mathrm{Nb}$. As discussed in Chapter 4 , the width of the $\theta-2 \theta \mathrm{XRD}$ peaks can be correlated to the X-ray coherence length by Equation 4.5 to estimate crystalline grain size. The films exhibit a slight changes from $30 \mathrm{~nm}$ grain size for the $30 \mathrm{~nm}$ thick film to $46 \mathrm{~nm}$ for the $600 \mathrm{~nm}$ thick film, evidencing the columnar growth typified by the Volmer-Weber growth mode during the nucleation stage.

\section{TEM Characterization}

The structure close to the interface was further investigated via TEM by collaborators C. Clavero (currently affiliated with the Thin Film Technology Group at Intermolecular Inc.) and K. C. Wong and A. D. Batchelor from North Carolina State University. Figure 5.11 shows a focused ion beam (FIB) prepared cross-sectional TEM image of the $\mathrm{Al}_{2} \mathrm{O}_{3}(11 \overline{2} 0) / \mathrm{Nb}(110)$ interface. A very sharp interface is present with good crystalline ordering on either side of the interface. Local analysis of the TEM images using FFT reveals that the interplane distance in the direction perpendicular to the surface for $\mathrm{Nb}$ mimics that of the underlying $\mathrm{Al}_{2} \mathrm{O}_{3}(11 \overline{2} 0)$ substrate $(0.237 \mathrm{~nm})$ across the entire thickness of the film $(\sim 15 \mathrm{~nm})$. These results agree well with a gradual, out-of-plane lattice parameter evolution observed with XRD, where the $30 \mathrm{~nm}$ thick $\mathrm{Nb}$ film was found to be strained by $1.25 \%$.

\subsubsection{Superconducting Measurements}

Magnetic susceptibility measurements were performed using SQUID magnetometry in both DC or AC configurations as described in Section 4.3. Figure 5.13 shows $\chi^{\prime}(\omega)$ (left column) and $\chi^{\prime \prime}(\omega)$ (right column) for the 30, 100, and $600 \mathrm{~nm}$ thick $\mathrm{Nb}$ films measured with an $\mathrm{AC}$ field of $3.5 \mathrm{Oe}$ and $1 \mathrm{~Hz}$ superimposed upon a 100 Oe DC field, oriented in-plane with the $\mathrm{Nb}$ film.

For the case of the 100 and $600 \mathrm{~nm}$ thick Nb film, a Type-II superconductor transition 


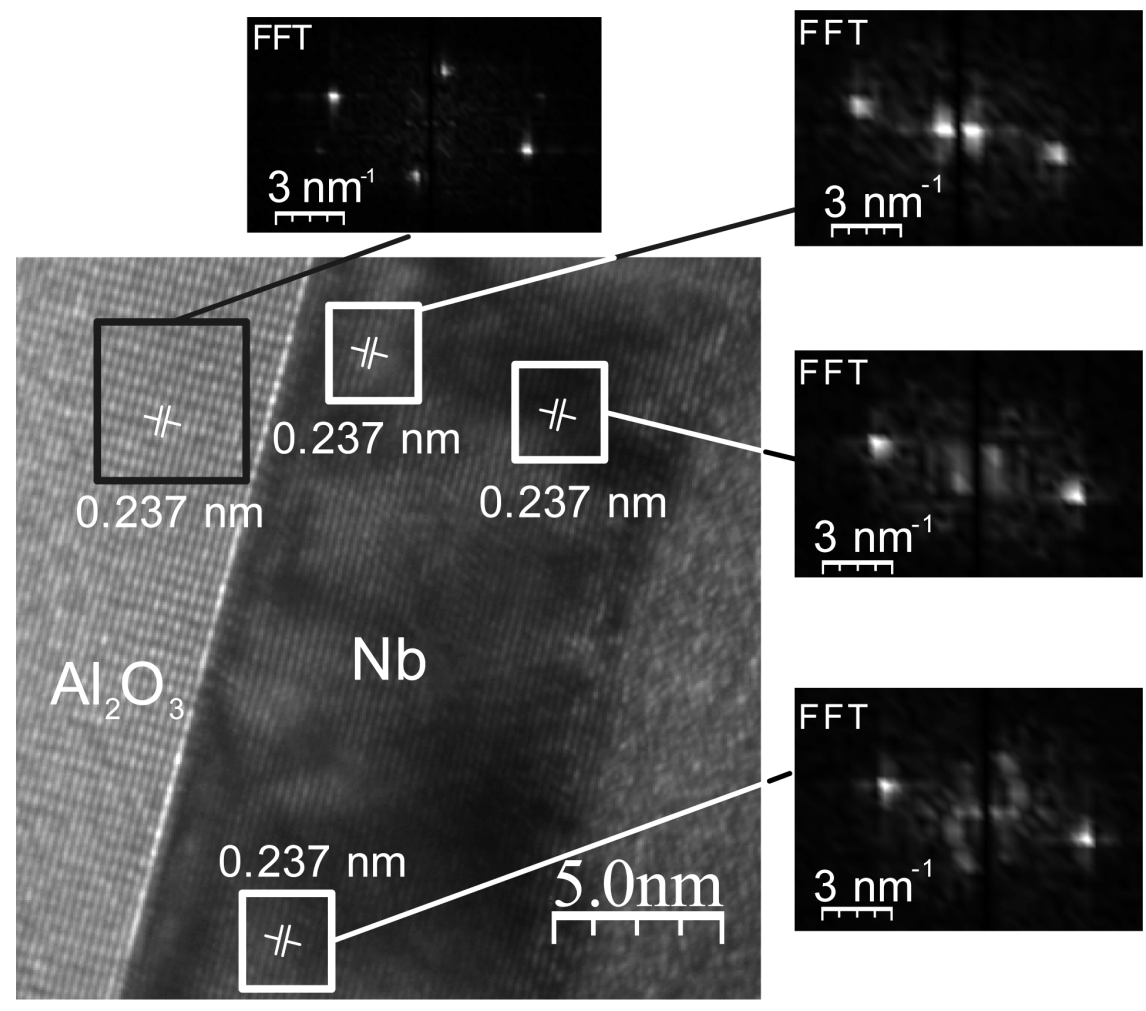

FIG. 5.11: TEM image of $\mathrm{Al}_{2} \mathrm{O}_{3}(11 \overline{2} 0) / \mathrm{Nb}(110)$ interface. The out of plane lattice parameter of the $\mathrm{Nb}$ film mimics that of the substrate across the entire thickness of the film $(\sim 15 \mathrm{~nm})$. Local 2D FFT maps for various regions are included. 
is observed at 8.75 and $8.7 \mathrm{~K}$, respectively. Note that the presence of a 100 Oe DC, applied to increase the AC susceptibility signal, also suppresses the measured transition temperature slightly. A separate low field DC measurement of $T_{C}$ on the $600 \mathrm{~nm}$ film yielded $9.29 \mathrm{~K}$ in agreement with the bulk value and a residual resistance ratio (RRR) of 97 [55]. The field used to probe the samples was chosen much smaller than the lower critical field $\mathrm{Hc1}$ for bulk Nb ( $1700 \mathrm{Oe})$ to minimize the impact on the transition temperatures of the superconducting films. This sharp drop of the transition temperature under a small applied field as compared to our field-free measurements hints at the presence of pinning sites correlated with the large surface roughness observed in these films. In addition, for the case of the thinner $30 \mathrm{~nm}$ thick $\mathrm{Nb}$ film, a $\chi^{\prime}(\omega)$ susceptibility transition with two steps can be clearly observed, accompanied by two peaks in the $\chi^{\prime \prime}(\omega)$ susceptibility at 7.64 and $8.08 \mathrm{~K}$.

The presence of two or more steps in the $\chi^{\prime \prime}(\omega)$ vs temperature dependence has been associated with the presence of grains and transport through grain boundaries in superconductors in previous studies. However, the $\mathrm{Nb} / a$-plane sapphire system investigated here exhibit similar grain sizes, ranging from $30 \mathrm{~nm}$ for the $30 \mathrm{~nm}$ thick $\mathrm{Nb}$ film to only $46 \mathrm{~nm}$ for the $600 \mathrm{~nm}$ film; thus, it is not expected that variations in intergrain contact to be related to $\mathrm{Nb}$ thickness in this case. Recall that RHEED and XRD analysis indicate that the Nb films are strained during the earliest stages of growth. Thus, the observed response for the $30 \mathrm{~nm}$ thick $\mathrm{Nb}$ film can be attributed to the presence of two predominant phases in the sample: (i) a first one with poor superconducting properties due to strain in the lattice and positioned closer to the interface with the substrate and (ii) a second one corresponding to the relaxed Nb layers that exhibit close to bulk Nb behavior.

As discussed in Section 2.5, a characteristic feature of superconducting thin films is that they exhibit unusually high $H_{C 1}$ when a magnetic field is applied in the plane of the film. The critical field in thin films is known to be dependent on the thickness and 


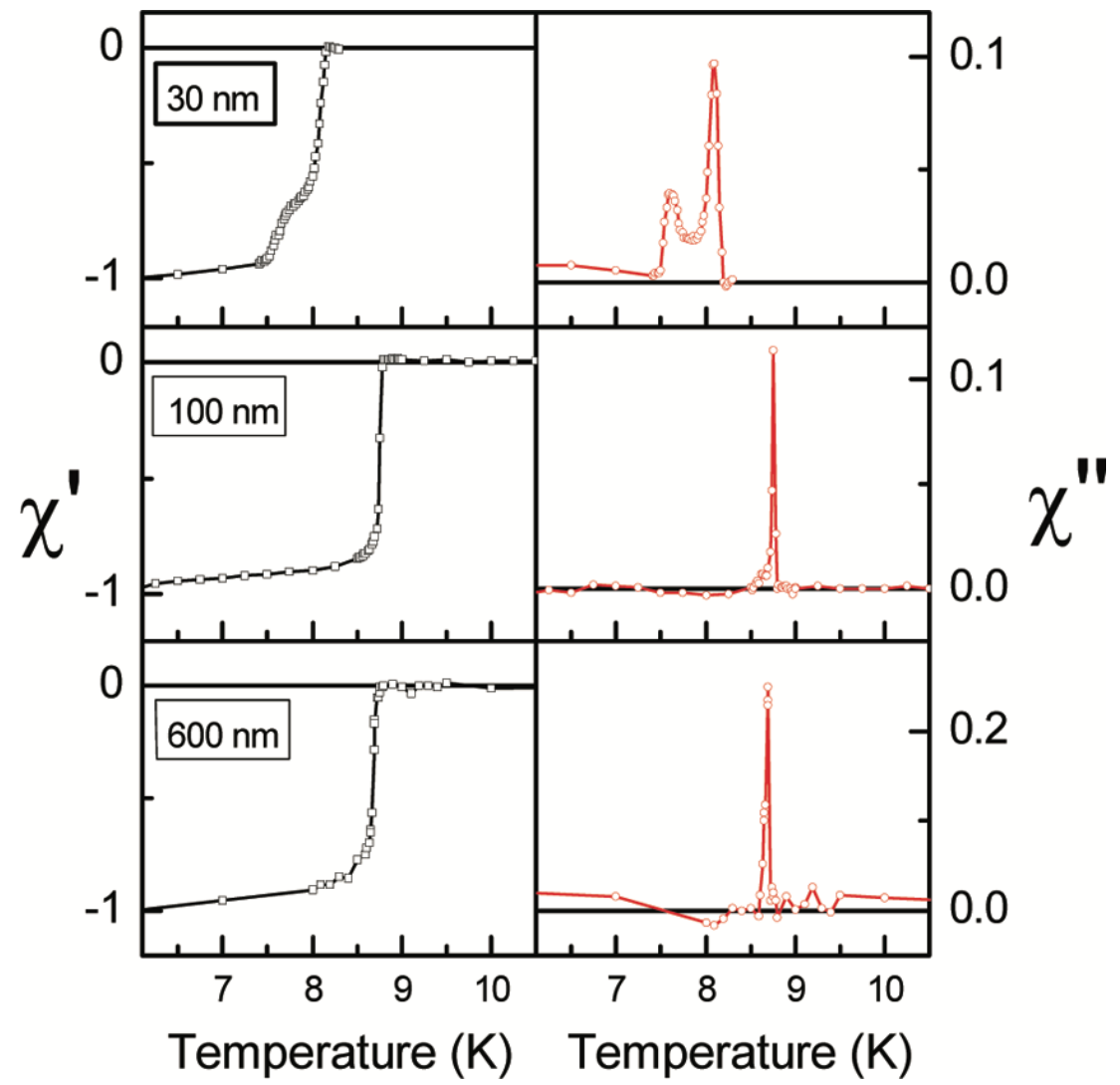

FIG. 5.12: AC susceptibility curves for $\mathrm{Nb}$ thin films (Left) Real $\chi^{\prime}(\omega)$ and (right) imaginary $\chi^{\prime \prime}(\omega)$ parts of the susceptibility for 30, 100 and $600 \mathrm{~nm} \mathrm{Nb}$ films grown on $a$-plane sapphire measured with an AC field of 3.5 Oe and $1 \mathrm{~Hz}$ superimposed upon a 100 Oe DC field. The stepped feature in the $30 \mathrm{~nm} \mathrm{Nb}$ film is associated with the strain in the earliest atomic layers. Note the relatively small 100 Oe DC field causes a slight depression of the transition temperature. 
also on nonlocal parameters such as the coherence length $\xi$ and the London penetration depth $\lambda_{L}$ of the material. The Ginzburg and Landau theory predicts a strong increase of the critical field in thin films, with extremely high critical fields at low thickness decaying exponentially to the bulk value around $600 \mathrm{~nm}$ films. Thus, we investigated such dependence by measuring the magnetization $\mathrm{M}$ in the superconducting state as a function of the externally applied in-plane magnetic field in the present Nb films, as shown Figure 5.13. The films show the typical behavior for Type-II superconductors, where the magnetization initially increases linearly with the externally applied field up to $H_{C 1}$, after which the film enters the vortex state causing deviation from the linear Meissner response as the applied field increases until the films enter a normal state above $H_{C 2}$.

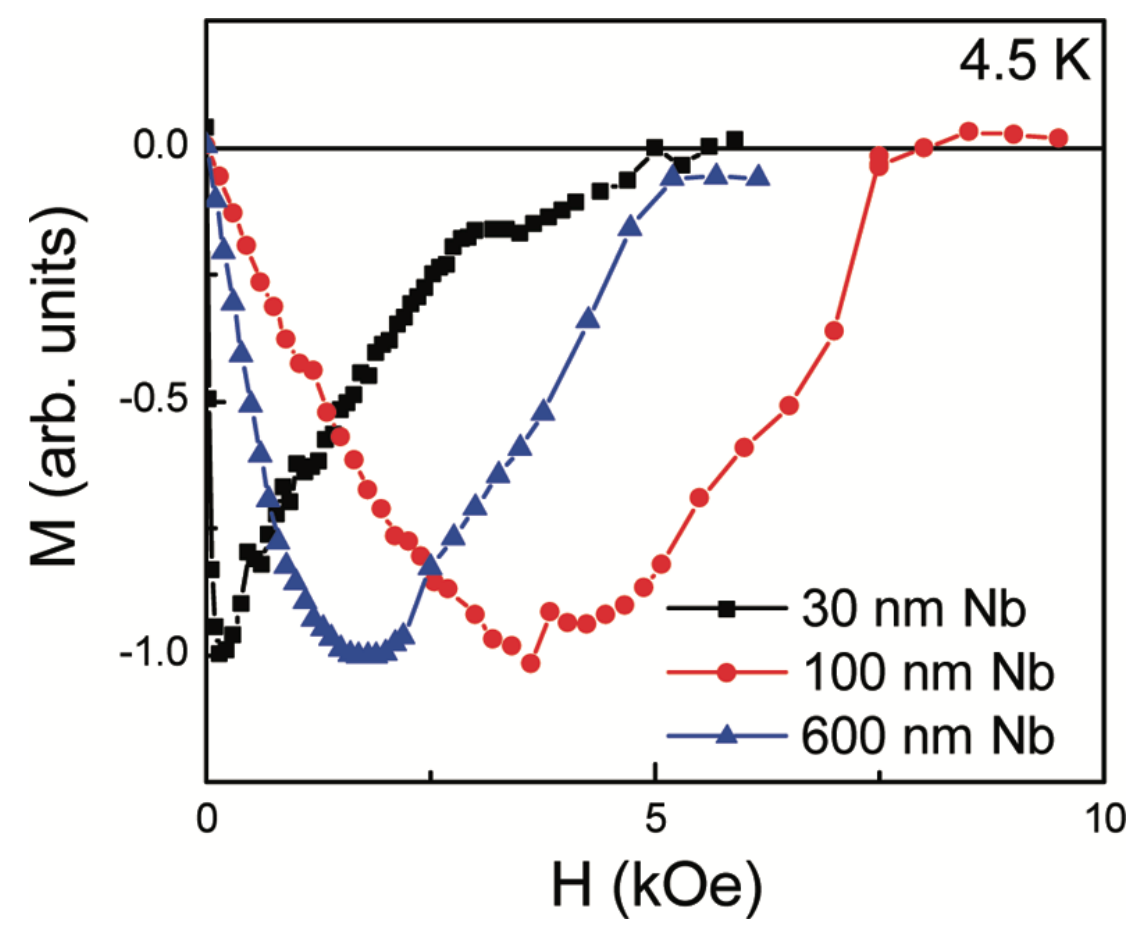

FIG. 5.13: In-plane magnetization vs. in-plane applied magnetic field for Nb films deposited on $a$-plane sapphire at $4.5 \mathrm{~K}$ for 30,100 and $600 \mathrm{~nm}$ thick Nb films. 
$H_{C 1}$ and $H_{C 2}$ results for the thickness series are summarized in Table 5.2. The 100 nm thick $\mathrm{Nb}$ sample exhibits an enhanced $H_{C 1}$ and $H_{C 2}$ as compared to a bulk Nb. The $30 \mathrm{~nm} \mathrm{Nb}$ film deviates from such trend and exhibits much lower critical fields than bulk; however, this result makes sense in the context of a suppressed $T_{C}$ (Figure 5.12), and further supports the presence of a phase exhibiting relatively poor superconducting properties at the interface with the substrate due to the presence of strain.

TABLE 5.2: Comparison of in-plane $H_{C 1}$ and $H_{C 2}$ values for a range of film thickness along with values for Bulk $\mathrm{Nb}$

\begin{tabular}{ccc}
\hline \hline $\begin{array}{c}\text { Nb thickness } \\
(\mathrm{nm})\end{array}$ & $\begin{array}{c}H_{C 1} \\
(\mathrm{Oe})\end{array}$ & $\begin{array}{c}H_{C 2} \\
(\mathrm{Oe})\end{array}$ \\
\hline 30 & 157 & 5000 \\
\hline 100 & 2000 & 8250 \\
\hline 600 & 1000 & 5182 \\
\hline Bulk & 1700 & 2400 \\
\hline \hline
\end{tabular}

A 2-fold surface morphological anisotropy is observed for films up to approximately $100 \mathrm{~nm}$ thick, coalescing towards uniaxial morphological anisotropy consisting of very elongated surface features for thicker films. RHEED and XRD characterization demonstrate the presence of an induced exotic hexagonal phase near the substrate, energetically more favorable for thicknesses up to $3 \mathrm{AL}$, followed by a strained bcc $\mathrm{Nb}(110)$ phase which relaxes progressively along the in-plane direction, approaching a bulk-like lattice parameter after $14 \mathrm{Nb}$ atomic layers. The strain relaxation was also investigated along the perpendicular direction using high angle XRD and cross sectional TEM. The superconducting properties of the initial strained layers are found to significantly suppressed when compared to fully relaxed $\mathrm{Nb}$ thin films. 


\section{3 $\quad \mathrm{MgB}_{2}$ Thin Films}

Thin film $\mathrm{MgB}_{2}$ is a promising material for technical improvements in SRF technology and applications. It has been predicted that for thin superconducting films — thickness less than the London penetration depth $\left(\lambda_{L} 120 \mathrm{~nm}\right.$ in the case of $\left.\mathrm{MgB}_{2}\right)$-the lower critical field $H_{C 1}$ can be enhanced with decreasing thickness. Thus, $\mathrm{MgB}_{2}$, with a high $T_{C}$ and relatively low $H_{C 1}$ value, as compared with $\mathrm{Nb}$, is a prime candidate for such SIS structures. Here we present our study on the microstructure, surface morphology, and superconducting properties on a thickness series of $\mathrm{MgB}_{2}$ thin films and correlate the effects of film thickness and surface morphology on $H_{C 1}$. The results of this study were first published in Reference $[9]$.

The success of SIS structures requires the intercalation of screening superconducting layers (with sufficiently large $T_{C}$ and $H_{C 1}$ ) with thin, insulating tunneling barriers in order to inhibit early vortex penetration and prevent early Q loss. For SRF cavities operating in the main $\mathrm{TM}(010)$ accelerating mode, the radio frequency magnetic field is oriented parallel to the cavity surface. From such geometrical considerations, the design of SIS superstructures may gain advantage from the superconducting thin film geometry — thin film superconductors whose thickness is less than the London penetration depth exhibit an enhancement of lower critical field $H_{C 1}$ and thus may remain in the Meissner state at much higher fields than their bulk counterparts $[5,14]$. Recent studies have demonstrated the viability of the SIS screening structure with $\mathrm{NbN} / \mathrm{MgO} / \mathrm{Nb}[56,37]$. Thus, $\mathrm{MgB}_{2}$ with its high critical temperature $(40 \mathrm{~K})$ and critical field larger than $\mathrm{Nb}$ (3500 Oe in powder samples) is an exciting candidate for novel, next-generation SRF materials [57]. In the present study, a thickness series of epitaxial $\mathrm{MgB}_{2} / \mathrm{c}$-plane sapphire thin films are characterized and the observed enhancement in the in-plane $H_{C 1}$ is correlated with microstructure and surface morphology. 


\subsection{1 $\quad \mathrm{MgB}_{2}$ Experimental Details}

As described in Section 2.5, recall that for superconducting films whose thickness, $d$, is less than the London penetration depth, $\lambda$, the in-plane lower critical field is described by

$$
B_{C 1}=\frac{2 \psi_{0}}{\pi d^{2}} \ln \frac{d}{(1.07) \xi}, \quad d<\lambda_{L}
$$

where $\psi_{0}$ is the flux quantum and the coherence length is $\xi$. Observation of this enhancement is extremely sensitive to alignment of the film surface with the applied magnetic field and is optimized for the ideal field parallel to the plane of the sample, i.e. in-plane geometry. High quality $\mathrm{MgB}_{2}$ films have been shown to possess London penetration depths $\left(\lambda_{L} \sim 120 \mathrm{~nm}\right)$ and critical fields $H_{C 1} \sim 280$ Oe; hence, films with $\mathrm{MgB}_{2}$ thickness less than $100 \mathrm{~nm}$ were chosen for this study $[57,58]$.

As described in Section 4.1.4, a thickness series of epitaxial (0001) $\mathrm{MgB}_{2} / \mathrm{c}$ Al2O3 (40, 60, 80 and $100 \mathrm{~nm})$ was fabricated using a hybrid physical-chemical vapor deposition (HPCVD) technique [32, 33]. Epitaxial $\mathrm{MgB}_{2}$ thin films produced by this method have been shown to exhibit excellent crystalline quality. Each $\mathrm{MgB}_{2}$ thin film in this study was subsequently capped with a passivating layer consisting of approximately $10 \mathrm{~nm}$ of $\mathrm{Au}$ in an effort to minimize the degradation of the active $\mathrm{MgB}_{2}$ surface during sample transportation in ambient conditions and during ex situ characterization.

Microstructure, surface morphology and superconducting performance were characterized for each sample in this thickness series. Film microstructure was examined using a four-circle, X-ray diffractometer (XRD) with a quasi-parallel monochromatic $\mathrm{Cu} K_{\alpha}$ $(\lambda=1.5406 \AA)$ beam optimized for thin films. The surface morphology of the gold capped 
$\mathrm{MgB}_{2}$ thin films were studied via ex situ atomic force microscopy (AFM) with corresponding software [41]. A Quantum Design magnetic properties measurement system (MPMS) superconducting quantum interference device (SQUID) magnetometer was used to characterize the superconducting properties of the thin film samples.

\subsubsection{Structural Characterization}

X-ray diffraction was used to determine lattice parameters, the mosaic structure of the epitaxial films, and also to give an estimate of grain size of the $\mathrm{MgB}_{2}$ films. The symmetric scan shown in Fig. 1(a), optimized on the $\mathrm{MgB}_{2}$ signal from each film, confirms the anticipated c-plane oriented epitaxial phase which has been reported elsewhere [32]. We also note the presence of a strong (111) Au signal corresponding to the capping layer.

The (0002) $\mathrm{MgB}_{2}$ peak corresponding to each film in the thickness series is presented in Fig. 1(b). The shifted position of the peak in each film is consistent with a strained epitaxial $\mathrm{MgB}_{2}$ film due to lattice mismatch with the underlying c-plane sapphire substrate. The symmetric scan for the $100 \mathrm{~nm}$ film also indicates the presence of a peak corresponding to a bulk-like or relaxed phase.

Lattice parameters, shown in Fig. 5.15 (c), were determined from diffraction peak location for each of the films. The contribution from the bulk-like phase of the $100 \mathrm{~nm}$ film is also included. As described previously in Section 4.2.2, for each film, grain sizes were estimated from the Scherrer Equation and mosaic structure was ascertained from rocking curves shown in Fig. 5.15 (d) [59]. While there is no obvious thickness trend in the microstructure, the $60 \mathrm{~nm}$ film exhibits larger grain size, better long-range order and a more relaxed lattice parameter as compared with the other samples from this series. 

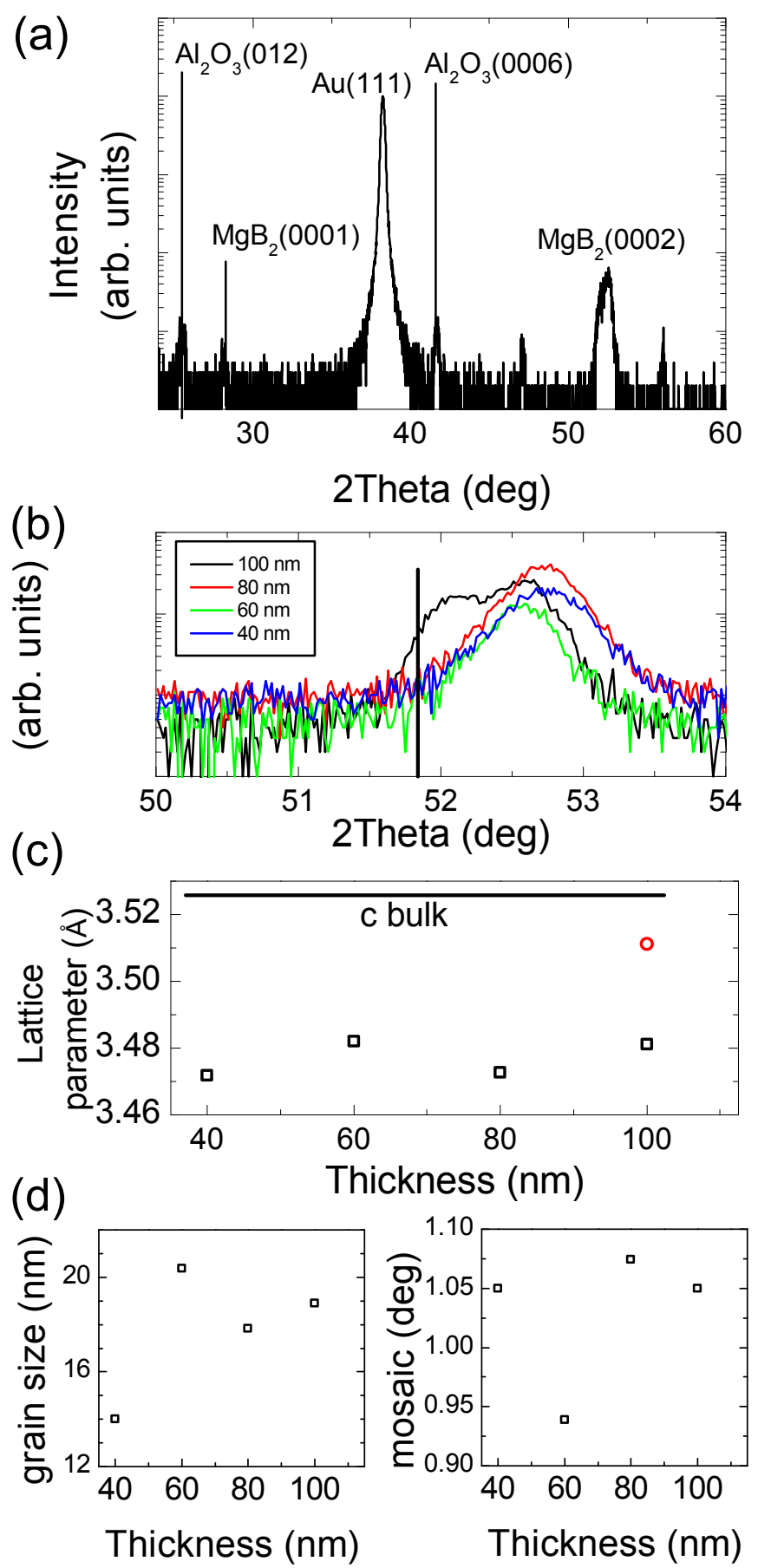

FIG. 5.14: X-ray diffraction of $\mathrm{MgB}_{2}$ on $c$-plane sapphire. (a) $\theta-2 \theta$ scan with diffraction peaks confirming the anticipated (0001) $\mathrm{MgB}_{2} /(0001) \mathrm{Al}_{2} \mathrm{O}_{3}$ epitaxy as well as presence of the $\mathrm{Au}$ capping layer; (b) symmetric scan about the $\mathrm{MgB}_{2}$ (0002) peak with a bold line indicating the bulk $\mathrm{MgB}_{2}$ peak position; (c) $\mathrm{MgB}_{2}$ lattice parameter vs. film thickness including bulk-like phase (in red) from the $100 \mathrm{~nm}$ film; and (d) (left) estimates of grain size vs. film thickness and (right) mosaic structure vs. film thickness. 


\subsubsection{Morphology of $\mathrm{MgB}_{2}$ Thin Films}

The surface morphology of the gold capped $\mathrm{MgB}_{2}$ films was examined via AFM. Representative topographical images for the 40 and $100 \mathrm{~nm}$ samples are presented in Fig. 5.15. The samples from this series are dominated by a coarse morphology, each with an RMS roughness $\sim 10 \mathrm{~nm}$. Consistent with the AFM observations, the surface roughness was sufficiently rough to prevent reconfirmation of film and capping layer nominal thickness via XRR methods.

\subsection{4 $\mathbf{H}_{C 1}$ Measurements on $\mathrm{MgB}_{2}$}

In order to test for the enhancement of the lower critical field $H_{C 1}$ expected in the thin film geometry, the samples were mounted such that that applied magnetic field was parallel to the film surface. Since perfect alignment with respect to the applied field cannot be assured with our current experimental setup, it is important to note that the measured parallel $H_{C 1}$ necessarily represents an underestimate of the critical field by virtue of the system geometry [14]. Some optimization with respect to sample alignment within the applied field is possible by rotating the sample azimuthally within the SQUID; thus the angular orientation allowing for a maximum Meissner response was taken to be "in alignment" with observed azimuthal deviations on the order of 100 Oe.

As discussed in Section 4.3, the method outlined by Bohmer et al. has advantages over the Meissner slope method which defines $H_{C 1}$ the field at which the isothermal M-H curve begins to deviate from the Meissner slope [47]. Namely, this procedure provides a more conservative measurement of the $H_{C 1}$ by early detection of vortex penetration that may not necessarily have a large effect on the magnetization curve due to flux pinning.

Fig. 5.16 shows the measured value of $H_{C 1}$ (taken at $4 \mathrm{~K}$ ) for each film examined plotted against a set of theoretical curves assuming a range of accepted values for $\mathrm{MgB}_{2}$ 

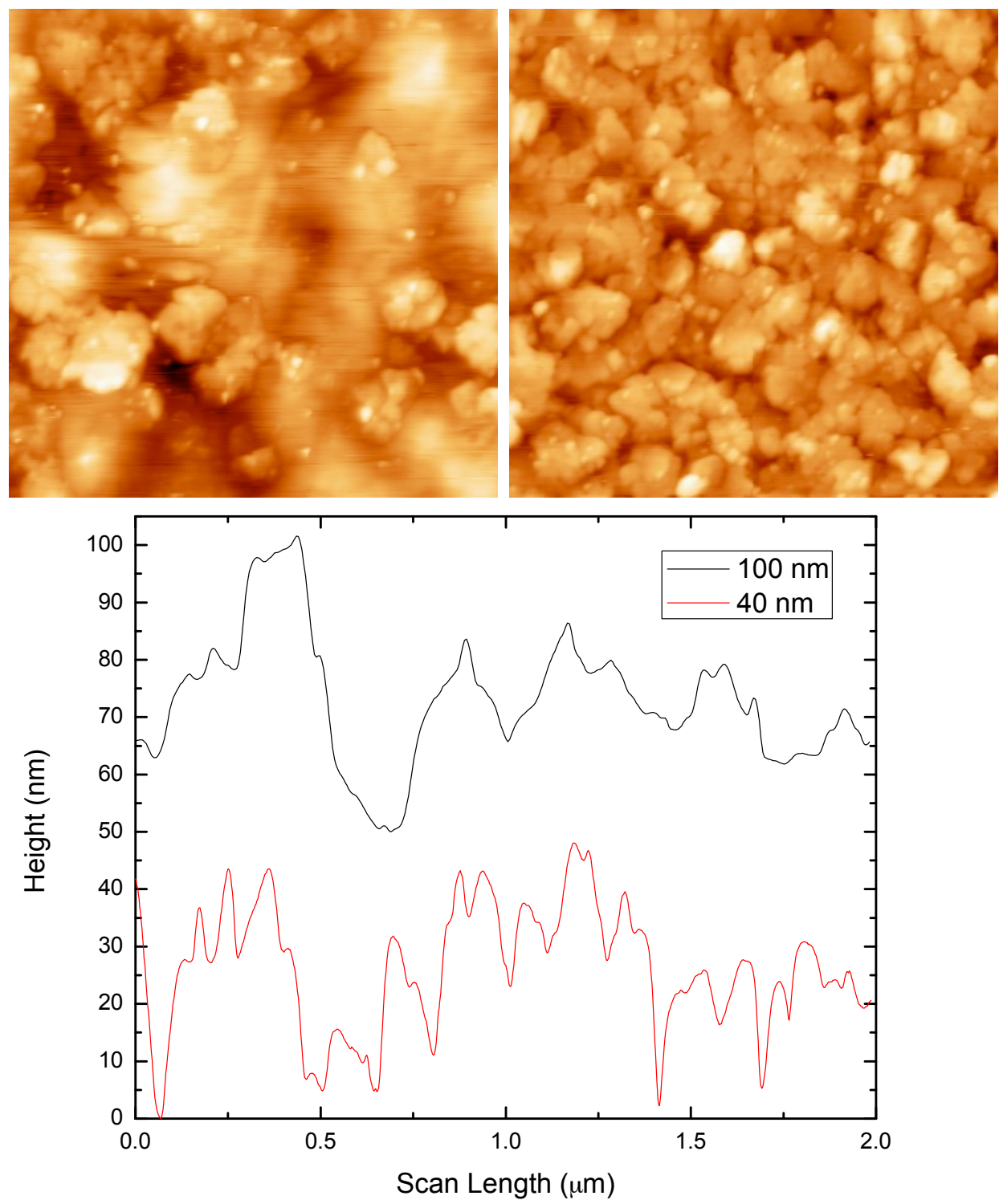

FIG. 5.15: AFM images of $\mathrm{MgB}_{2}$ thin films. (Top left) $2 \times 2$ micron AFM image of a $10 \mathrm{~nm}$ $\mathrm{Au} / 100 \mathrm{~nm} \mathrm{MgB}{ }_{2}$ thin film and (top right) 2 x 2 micron AFM image of a $10 \mathrm{~nm} \mathrm{Au} / 40 \mathrm{~nm}$ $\mathrm{MgB}_{2}$ thin film. (Bottom) Representative offset line scans illustrating relative feature profiles. Films from this series demonstrate coarse features relative to nominal thickness. The z-scale for each false color AFM image is $100 \mathrm{~nm}$ with light portions corresponding to taller features. 
coherence lengths $[57,60,61]$. The thin films with the largest grain size and more bulk like lattice parameters (the $60 \mathrm{~nm}$ and $100 \mathrm{~nm}$ films) appear to exhibit well-correlated microstructure with the observed enhancement of $H_{C 1}$. The relatively poor $H_{C 1}$ performance of the 40 and $80 \mathrm{~nm}$ films with respect to the expected theoretical predictions are consistent with the observed microstructure and, particularly in the case of the $40 \mathrm{~nm}$ film, may be further hampered by the coarse surface morphology observed in this case. It is worth stressing here that these $H_{C 1}$ values represent an underestimate of the lower critical field since stringent alignment is limited with our current MPMS configuration.

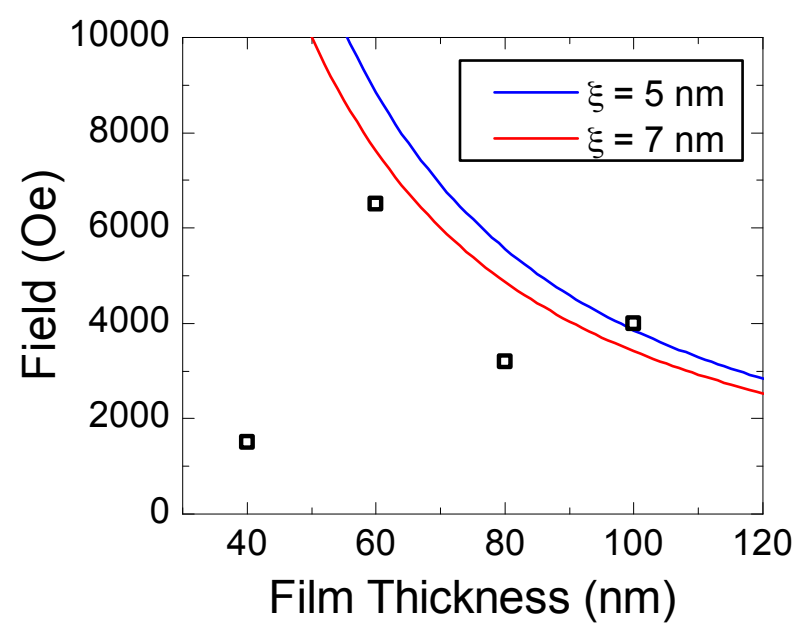

FIG. 5.16: Measured $\mathrm{H}_{C 1}$ vs. $\mathrm{MgB}_{2}$ film thickness (taken at $4 \mathrm{~K}$ ). Solid lines represent expected theoretical curves for lower critical field enhancement for particular coherence lengths. Associated error for all critical field measurements are on the order of $\sim 100$ Oe and therefore not displayed on the plot.

\subsection{5 $\quad \mathrm{MgB}_{2}$ Summary}

The observed superconducting properties for a thickness series of $\mathrm{MgB}_{2}$ films deposited on c-plane sapphire correlate well with ascertained microstructure and surface morphology observed in these thin films. The observation of coarse morphology, perhaps due in part to the gold capping layer, may be detrimental to the superconducting performance of the 
thinnest film (40 nm); however, we do observe a significant enhancement in $H_{C 1}$ for the $60 \mathrm{~nm}$ film and the overall trend roughly follows the theoretical curve. 


\section{CHAPTER 6}

\section{Conclusion and Outlook}

The bulk of this dissertation is concerned with the growth and characterization of $\mathrm{Nb}$ and $\mathrm{MgB}_{2}$ epitaxially grown on insulating substrates for SRF applications. In the case of epitaxial $\mathrm{Nb} / \mathrm{MgO}(001)$, we have demonstrated that there are two attainable epitaxial registries - $\mathrm{Nb}(110) / \mathrm{MgO}(001)$ with two possible azimuthal crystalline domains corresponding to $\mathrm{Nb}[110] \| \mathrm{MgO}[100]$ and $\mathrm{Nb}[110] \| \mathrm{MgO}[010]$ as well as $\mathrm{Nb}(001) / \mathrm{MgO}(001)$ where $\mathrm{Nb}[100] \| \mathrm{MgO}[100]$ — which may be selected and stabilized with careful control of substrate conditions and sputter deposition rates. Additional scaling studies correlating the interfacial surface roughness with varying film thickness indicate that the evolution of the surface morphology in each $\mathrm{Nb} / \mathrm{MgO}$ registry proceeds according to a universality class within a general dynamic scaling formalism. In practice, this mode of analysis may be useful to predict the surface morphology of films grown at intermediate film thickness. Critical field measurements on each epitaxial series suggests that $\mathrm{Nb}(001) / \mathrm{MgO}(001)$ features higher, more favorable $H_{C 1}$ values as compared with $\mathrm{Nb}(110) / \mathrm{MgO}(001)$.

The evolution of strain as a function of thickness in epitaxial $\mathrm{Nb} / \mathrm{Al}_{2} \mathrm{O}_{3}$ in the early stages of growth has also been presented. A novel, strained hexagonal phase of $\mathrm{Nb}$ has 
been identified in the first few monolayers of the $\mathrm{Nb} / \mathrm{Al}_{2} \mathrm{O}_{3}$ system. Superconducting measurements on these samples indicate a strong dependence on film thickness, where the thinest samples are characterized by a suppressed $\mathrm{T}_{C}$ and $\mathrm{H}_{C 1}$.

Our $\mathrm{H}_{C 1}$ measurements on epitaxial thin film $\mathrm{MgB}_{2}$ samples experimentally confirm (for the first time)an anticipated $\mathrm{H}_{C 1}$ enhancement, particular to thin film geometries, for the first time in thin film $\mathrm{MgB}_{2}$. The results of this study are encouraging and suggest that the SIS multilayer approach may indeed benefit with well tailored thin film superconducting compounds.

There remains considerable interest in developing SIS multilayers and thin film coated SRF cavities to push state of the art SRF technology beyond intrinsic material limits. While steady progress has been made over the decades, as a practical matter, the realization of SIS multilayer structures is hampered by significant challenges, such as faithfully producing (and reproducing) high-quality superconducting thin films under ideal, commercial substrate conditions by traditional deposition methods. As was discussed in Chapter 3, control of the structure and surface morphology of the first layer in the SIS superstructure is paramount if subsequent multilayers are to be successful. There are additional challenges present in the separate but important industrial scaling problem of achieving conformal growth of viable SIS multilayers within the cylindrical geometry of an SRF cavity. This degree of control over thin film growth can only be attained through careful studies, like those presented here, seeking to understand and access the relevant growth mechanisms leading to superconducting thin films with good structure, morphology and superconducting characteristics.

There is promising work being done with energetic deposition techniques, such electron cyclotron resonance (ECR) plasma deposition and high-power impulse magnetron sputtering (HiPIMS) at Jlab and elsewhere in the SRF community. Greater control of the kinetics of deposition grants access to growth modes hitherto unachievable by lower en- 
ergy deposition techniques. IBAD and other plasma-assisted deposition techniques allow for the possibility of modifying the evolution of the surfaces, during growth, simultaneously tailoring dense, crystalline epitaxial films with minimized surface features. There are many research directions left to explore for thin-film SRF coatings. Regardless of the particular path forward, the characterization techniques, structure-property correlations and general dynamic scaling and roughness analysis featured in this dissertation are generally amenable to new materials, deposition techniques and thus can serve as a platform for comparison for future work. 


\section{BIBLIOGRAPHY}

[1] L. Lilje et al., in Proc. of EPAC (2004).

[2] A. Schwettman, P. B. Wilson, J. M. Pierce, and W. M. Fairbank, International Advances in Cryogenic Engineering 10, 88 (1965).

[3] H. Padamsee, J. Knobloch, and T. Hays, RF Superconductivity for Accelerators (WILEY-VCH, 2008), 2nd ed.

[4] N. Valles, R. Eichhorn, F. Furuta, M. Gi, D. Gonnella, V. Ho, G. Hoffstaetter, M. Liepe, T. O'Connell, S. Posen, et al., Proc. of the SRF 2013 conference, Paris pp. 300-304 (2013).

[5] A. Gurevich, Appl. Phys. Lett. 88, 012511 (2006).

[6] S. Posen and M. Liepe, Proceeding of PAC, Pasadena, CA USA pp. 754-758 (2013).

[7] D. B. Beringer, W. M. Roach, C. Clavero, C. E. Reece, and R. A. Lukaszew, Phys. Rev. ST Accel. Beams 16, 022001 (2013).

[8] C. Clavero, D. B. Beringer, W. M. Roach, J. R. Skuza, K. C. Wong, A. D. Batchelor, C. E. Reece, and R. A. Lukaszew, Cryst. Growth Des. 12, 2588 (2012).

[9] D. B. Beringer, C. Clavero, T. Tan, X. X. Xi, W. M. Roach, and R. A. Lukaszew, IEEE Trans. Appl. Supercond. 23, 7500604 (2013). 
[10] D. B. Beringer, W. M. Roach, C. Clavero, C. E. Reece, and R. A. Lukaszew, J. Appl. Phys. 114, 223502 (2013).

[11] W. M. Roach, PhD in applied science, The College of William and Mary, Williamsburg Virginia, USA (2014), uMI Number: 3580414.

[12] J. File and R. G. Mills, Phys. Rev. Lett. 10, 93 (1963).

[13] E. Maxwell, Phys. Rev. 78, 477 (1950).

[14] G. Stejic, A. Gurevich, E. Kadyrov, D. Christen, R. Joynt, and D. C. Larbalestier, Phys. Rev. B 49, 1274 (1994).

[15] U. Penner, H. Rucker, and I. N. Yassievich, Semicond. Sci. Technol. 13, 709 (1998).

[16] A. Rosengren, L. M. Bjursten, N. Danielsen, H. Persson, and M. Kober, J. Mater. Sci. : Mater. Med. 10, 75 (1999).

[17] S. Calatroni, J. Physica C 441, 95 (2006).

[18] M. Ohring, The Materials Science of Thin Films, Referex Engineering (Academic Press, 1992), ISBN 9780125249904, URL https://books.google.com/books?id=_oC1zwLnoRwC.

[19] J. A. Thornton, J. Vac. Sci. Technol. 11, 666 (1974).

[20] A. Anders, Thin Solid Films 518, 4087 (2010).

[21] A.-L. Barabasi and H. E. Stanley, Fractal Concepts in Surface Growth (Cambridge University Press, Cambridge, 1995).

[22] X. Ge and K. Morgenstern, Phys. Rev. B 85, 045417 (2012).

[23] F. C. Frank and J. H. van der Merwe, Proc. R. Soc. London, Ser. A 198, 205 (1949). 
[24] M. Volmer and A. Weber, Z. Phys. Chem. 119, 277 (1926).

[25] B. Wiedenhorst, C. Hofener, Y. Lu, J. Klein, L. Alff, R. Gross, B. H. Freitag, and W. Mader, Appl. Phys. Lett. 74, 3636 (1999).

[26] B. B. Mandelbrot, The Fractal Geometry of Nature (W. H. Freeman and Company, 1977).

[27] T. A. Witten and L. M. Sander, Phys. Rev. Lett. 47, 1400 (1981), URL http://link.aps.org/doi/10.1103/PhysRevLett.47.1400.

[28] T. Vicsek, M. Shlesinger, and M. Matsushita, Fractals in Natural Sciences (World Scientific, 1994), ISBN 9789810216245, URL https: //books.google. com/books?id=o01xQgAACAAJ.

[29] F. Family and T. Vicsek, J. Phys. A 18, L75 (1985).

[30] J. J. Ramasco, J. M. Lopez, and M. A. Rodriquez, Phys. Rev. Lett. 84, 2199 (2000).

[31] B. C. Mohanty, H. R. Choi, and Y. W. Cho, EPL-Europhysics Lett. 93, 26003 (2011).

[32] C. Zhuang, T. Tan, Y. Wang, S. Bai, X. Ma, H. Yang, G. Zhang, Y. He, H. Wen, X. X. Xi, et al., Supercond. Sci. Technol. 22, 025002 (2009).

[33] C. Zhuang, K. Chen, J. M. Redwing, Q. Li, and X. X. Xi, Supercond. Sci. Technol. 23, 055004 (2010).

[34] P. J. Kelly and R. D. Arnell, Vacuum 56, 159 (2000).

[35] A. Anders, Thin Solid Films 502, 22 (2006).

[36] W. M. Roach, D. B. Beringer, J. R. Skuza, W. A. Oliver, C. Clavero, C. E. Reece, and R. A. Lukaszew, Phys. Rev. ST Accel. Beams 15, 062002 (2012). 
[37] W. M. Roach, D. B. Beringer, Z. Li, C. Clavero, and R. A. Lukaszew, IEEE Trans. Appl. Supercond. 23, 8600203 (2013).

[38] Z.-K. Liu, D. G. Schlom, Q. Li, and X. X. Xi, Applied Physics Letters 78, $3678 \quad$ (2001), URL http://scitation.aip.org/content/aip/journal/apl/78/23/10.1063/1.1376145.

[39] A. Ichimiya and P. Cohen, Reflection High-Energy Electron Diffraction (Cambridge University Press, 2004), ISBN 9780521453738, URL https://books.google.com/books?id=AUVbPerNxTcC.

[40] C. Xu, H. Tian, C. E. Reece, and M. J. Kelley, Phys. Rev. ST Accel. Beams 14, $123501(2011)$.

[41] I. Horcas, R. Fernandez, J. M. Gomez-Rodriguez, J. Colchero, J. Gomez-Herrero, and A. M. Baro, Rev. Sci. Instrum. 78, 013705 (2007).

[42] T. E. Hutchinson, J. Appl. Phys. 36, 270 (1965).

[43] M. Morcrette, A. Gutierrez-Llorente, W. Seiler, J. Perriere, A. Laurent, and P. Barboux, J. Appl. Phys. 88, 5100 (2000).

[44] J. Narayan, K. Dovidenko, A. K. Sharma, and S. Oktyabrsky, J. Appl. Phys. 84, 2597 (1998).

[45] M. A. Auger, L. Vazquez, R. Cuerno, M. Castro, M. Jergel, and O. Sanchez, Phys. Rev. B 73, 045436 (2006).

[46] M. Krishnan, E. Valderrama, C. James, X. Zhao, J. Spradlin, A.-M. V. Feliciano, L. Phillips, C. E. Reece, K. Seo, and Z. H. Sung, Phys. Rev. Accel. Beams 15, 032001 (2012). 
[47] C. Bohmer, G. Brandstatter, and H. W. Weber, Supercond. Sci. Technol. 10, A1 (1997).

[48] A. F. Mayadas, R. B. Laibowitz, and J. J. Cuomo, J. Appl. Phys. 43, 1287 (1972).

[49] A. R. Wildes, J. Mayer, and K. T. Brohl, Thin Solid Films 401, 7 (2001).

[50] J. H. Claasen, S. A. Wolf, S. B. Qadri, and L. D. Jones, J. Cryst. Growth 81, 557 (1987).

[51] J. E. Grier, M. L. Jenkins, A. K. Petford-Long, R. C. C. Ward, and M. R. Wells, Thin Solid Films 385, 94 (2000).

[52] J. W. Matthews and A. E. Blakeslee, J. Cryst. Growth 27, 188 (1974).

[53] V. Oderno, C. Dufour, K. Dumesnil, A. M. P. Mangin, and G. Marchal, Philos. Mag. Lett. 78, 419 (1998).

[54] G. L. Zhou and C. P. Flynn, Phys. Rev. B 59, 7860 (2010).

[55] X. Zhao, L. Phillips, C. E. Reece, K. Seo, M. Krishnan, and E. Valderrama, J. Appl. Phys. 110, 033523 (2011).

[56] C. Z. Antoine, S. Berry, S. Bouat, J.-F. Jacquot, J.-C. Villegier, G. Lamura, and A. Gurevich, Phys. Rev. ST Accel. Beams 13, 121001 (2010).

[57] X. X. Xi, Supercond. Sci. Technol. 22, 043001 (2009).

[58] A. A. Golubov, A. Brinkman, O. V. Dolgov, J. Kortus, and O. Jepsen, Phys. Rev. B 66, $054524(2002)$.

[59] A. Patterson, Phys. Rev. 56, 978 (1939). 
[60] B. B. Jin, T. Dahm, C. Iniotakis, A. I. Gubin, E.-M. Choi, H. J. Kim, S.-I. Lee, W. N. Kang, S. F. Wang, Y. L. Zhou, et al., Supercond. Sci. Technol. 15, L1 (2005).

[61] M. Zehetmayer, M. Eisterer, J. Jun, S. M. Kasakov, J. Karpinski, A. Wisniewski, and H. W. Weber, Phys. Rev. B 66, 052505 (2002). 